\title{
Higgs Boson Searches at Hadron Colliders
}

\author{
Volker Büscher and Karl Jakobs \\ Physikalisches Institut, Universität Freiburg \\ Hermann-Herder-Str. 3, 79104 Freiburg, Germany \\ buescher@fnal.gov \\ karl.jakobs@uni-freiburg.de
}

February 18, 2005

\begin{abstract}
The investigation of the dynamics responsible for electroweak symmetry breaking is one of the prime tasks of experiments at present and future colliders. Experiments at the Tevatron $p \bar{p}$ Collider and at the CERN Large Hadron Collider (LHC) must be able to discover a Standard Model Higgs boson over the full mass range as well as Higgs bosons in extended models. In this review, the discovery potential for the Standard Model Higgs boson and for Higgs bosons in the Minimal Supersymmetric extension is summarized. Emphasis is put on those studies which have been performed recently within the experimental collaborations using a realistic simulation of the detector performance. This includes a discussion of the search for Higgs bosons using the vector boson fusion mode at the LHC, a discussion on the measurement of Higgs boson parameters as well as a detailed review of the MSSM sector for different benchmark scenarios. The Tevatron part of the review also contains a discussion of first physics results from data taken in the ongoing Run II.
\end{abstract}

\section{Introduction}

Over the next decade, hadron colliders will play an important role in the investigation of fundamental questions of particle physics. While the Standard Model of electroweak 1$]$ and strong 2 interactions is in excellent agreement with the numerous experimental measurements, the dynamics responsible for electroweak symmetry breaking are still unknown. Within the Standard Model, the Higgs mechanism 3 is invoked to break the electroweak symmetry. A doublet of complex scalar fields is introduced of which a single neutral scalar particle, the Higgs boson, remains after symmetry breaking. 4 Many extensions of this minimal version of the Higgs sector have been proposed, including a scenario with two complex Higgs doublets as realized in the Minimal Supersymmetric Standard Model (MSSM)!

Within the Standard Model, the Higgs boson is the only particle that has not been discovered so far. The direct search at the $e^{+} e^{-}$collider LEP has led to a lower bound on its mass of $114.4 \mathrm{GeV} / \mathrm{c}^{2}{ }^{6]}$ Indirectly, high precision electroweak data constrain the mass of the Higgs boson via their sensitivity to loop corrections. Assuming the overall validity of the Standard Model, a global fit 7 to all electroweak data leads to $m_{H}=114_{-45}^{+69} \mathrm{GeV} / \mathrm{c}^{2}$. On the basis of the present theoretical knowledge, the Higgs sector in the Standard Model remains largely unconstrained. While there is no direct prediction for the mass of the Higgs boson, an upper limit of $\sim 1 \mathrm{TeV} / \mathrm{c}^{2}$ can be inferred from unitarity arguments $\underline{8}$

Further constraints can be derived under the assumption that the Standard Model is valid only up to a cutoff energy scale $\Lambda$, beyond which new physics becomes relevant. Requiring that the electroweak vacuum is stable and that the Standard Model remains perturbative allows to set upper and lower bounds on the Higgs boson mass $\frac{910}{}$ For a cutoff scale of the order of the Planck mass, the Higgs boson mass is required to be in the range $130<m_{H}<190 \mathrm{GeV} / \mathrm{c}^{2}$. If new physics appears at lower mass scales, the bound becomes weaker, e.g., for $\Lambda=1 \mathrm{TeV}$ the Higgs boson mass is constrained to be in the range $50<m_{H}<800 \mathrm{GeV} / \mathrm{c}^{2}$.

The Minimal Supersymmetric Standard Model contains two complex Higgs doublets, leading to five physical Higgs bosons after electroweak symmetry breaking: three neutral (two CP-even $h, H$ 
and one CP-odd $A$ ) and a pair of charged Higgs bosons $H^{ \pm}$. At tree level, the Higgs sector of the MSSM is fully specified by two parameters, generally chosen to be $m_{A}$, the mass of the CPodd Higgs boson, and $\tan \beta$, the ratio of the vacuum expectation values of the two Higgs doublets. Radiative corrections modify the tree-level relations significantly. This is of particular interest for the mass of the lightest CP-even Higgs boson, which at tree level is constrained to be below the mass of the $Z$ boson. Loop corrections are sensitive to the mass of the top quark, to the mass of the scalar particles and in particular to mixing in the stop sector. The largest values for the mass of the Higgs boson $h$ are reached for large mixing, characterized by large values of the mixing parameter $X_{t}:=A_{t}-\mu \cot \beta$, where $A_{t}$ is the trilinear coupling and $\mu$ is the Higgs mass parameter. If the full one-loop and the dominant two-loop contributions are included,1112 the upper bound on the mass of the light Higgs boson $h$ is expected to be around $135 \mathrm{GeV} / \mathrm{c}^{2}$. While the light neutral Higgs boson may be difficult to distinguish from the Standard Model Higgs boson, the other heavier Higgs bosons are a distinctive signal of physics beyond the Standard Model. The masses of the heavier Higgs bosons $H, A$ and $H^{ \pm}$are often almost degenerate.

Direct searches at LEP have given lower bounds of 92.9 (93.3) $\mathrm{GeV} / \mathrm{c}^{2}$ and $93.4(93.3) \mathrm{GeV} / \mathrm{c}^{2}$ on the masses of the lightest CP-even Higgs boson $h$ and the CP-odd Higgs boson $A$ within the $m_{h}$-max (no-mixing) scenario. 13 In those scenarios, the mixing parameter in the stop sector is set to values of $X_{t}=2 \mathrm{TeV} / \mathrm{c}^{2}$ and $X_{t}=0$, respectively. Given the LEP results, the $\tan \beta$ regions of 0.9 $<\tan \beta<1.5$ and $0.4<\tan \beta<5.6$ are excluded at $95 \%$ confidence level for the $m_{h}$-max and the no-mixing scenarios, respectively 13$]$ However, it should be noted that the exclusions in $\tan \beta$ depend critically on the exact value of the top-quark mass. In the LEP analysis $m_{t}=179.3 \mathrm{GeV} / \mathrm{c}^{2}$ has been assumed. With increasing top mass the theoretical upper bound on $m_{h}$ increases and hence the exclusion in $\tan \beta$ decreases, e.g., for $m_{t}$ of about $183 \mathrm{GeV} / \mathrm{c}^{2}$ or higher the exclusions in $\tan \beta$ vanish.

The charged Higgs boson mass is related to $m_{A}$ via the tree-level relation $m_{H^{ \pm}}^{2}=m_{W}^{2}+m_{A}^{2}$ and is less sensitive to radiative corrections. 14 Direct searches for charged Higgs bosons in the decay modes $H^{ \pm} \rightarrow \tau \nu$ and $H^{ \pm} \rightarrow c s$ have been carried out at LEP, yielding a lower bound of $78.6 \mathrm{GeV} / \mathrm{c}^{2}$ on $m_{H^{ \pm}}$independent of the $H^{ \pm} \rightarrow \tau \nu$ branching ratio!15 At the Tevatron, the CDF and D $\varnothing$ experiments have performed direct and indirect searches for the charged Higgs boson through the process $p \bar{p} \rightarrow t \bar{t}$ with at least one top quark decaying via $t \rightarrow H^{ \pm} b$. These searches have excluded the small and large $\tan \beta$ regions for $H^{ \pm}$masses up to $\sim 160 \mathrm{GeV} / \mathrm{c}^{2}[16]$ Other experimental bounds on the charged Higgs boson mass can be derived using processes where the charged Higgs boson enters as a virtual particle. For example, the measurement of the $b \rightarrow s \gamma$ decay rate allows to set indirect limits on the charged Higgs boson mass, $\frac{17}{17}$ which, however, are strongly model dependent 18

The high collision energy of the Fermilab Tevatron $p \bar{p}$ collider and the CERN Large Hadron Collider (LHC) allow to extend the search for Higgs bosons into unexplored mass regions. In particular the experiments at the LHC have a large discovery potential for Higgs bosons in both the Standard Model and in the MSSM over the full parameter range. Should the Higgs boson be light, i.e., have a mass in the range favoured by the precision electroweak measurements, also the experiments at the Tevatron will have sensitivity for discovery. The mass range accessible depends, however, critically on the integrated luminosity that can be collected.

In this article, the potential for Higgs boson searches at these hadron colliders is reviewed, focussing on the investigation of the Higgs sectors in the Standard Model and in the MSSM. This subject is discussed in the literature in numerous notes and publications. For this review the main emphasis is put on studies which have been performed recently within the experimental collaborations using a realistic simulation of the detector performance. This includes a discussion of the search for Higgs bosons using the vector boson fusion mode at the LHC, a discussion of the measurement of Higgs boson parameters at the LHC as well as a detailed review of analyses within the MSSM for different benchmark scenarios. For the Tevatron, first results based on the data taken in the ongoing Run II are discussed in addition to a review of the Monte Carlo studies and projections.

In Section 2, Higgs boson production and decay processes and the status of the calculation of higher order QCD corrections are presented. The experimental scenarios at the Tevatron and at the LHC are briefly discussed in Section 3. The current status and expected performance in the search for both a Standard Model Higgs boson and MSSM Higgs bosons at the Tevatron is presented in Section 4. The LHC potential for discovery of a Standard Model Higgs boson, for the measurement of its parameters and for the discovery of MSSM Higgs bosons in various benchmark scenarios is 

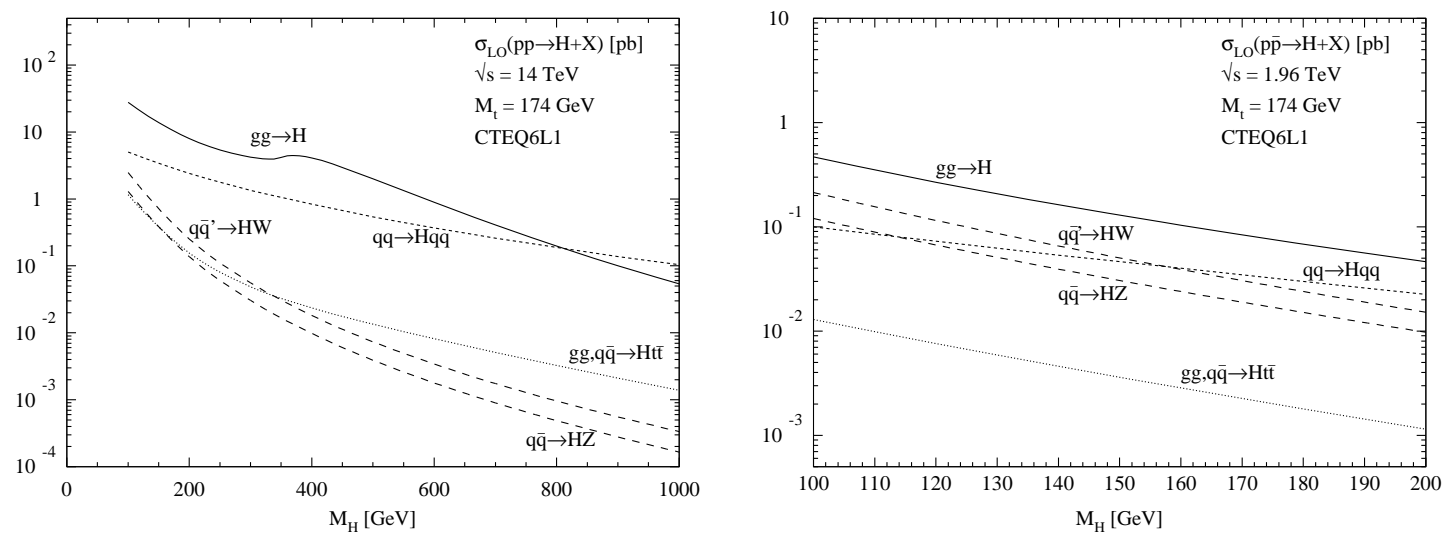

Figure 1: Leading order production cross sections for a Standard Model Higgs boson as a function of the Higgs boson mass at the LHC $14 \mathrm{TeV}$ pp collider (left) and at the $1.96 \mathrm{TeV}$ Tevatron $\bar{p}$ collider (right). In the cross section calculation the CTEQ6L1 structure function parametrization has been used. (The calculations have been performed by M. Spira, Ref. 19)

summarized in Sections 5 to 7.

\section{Higgs Bosons at Hadron Colliders}

\subsection{Higgs boson production}

At hadron colliders, Higgs bosons can be produced via four different production mechanisms:

- gluon fusion, $g g \rightarrow H$, which is mediated at lowest order by a heavy quark loop;

- vector boson fusion (VBF), $q q \rightarrow q q H$;

- associated production of a Higgs boson with weak gauge bosons, $q q \rightarrow W / Z H$ (Higgs Strahlung, Drell-Yan like production);

- associated Higgs boson production with heavy quarks, $g g, q q \rightarrow t t H, g g, q q \rightarrow b b H$ (and $g b \rightarrow b H)$.

The lowest order production cross sections for the four different processes are shown in Fig. \for both the Tevatron and the LHC collider as a function of the Higgs boson mass. $\frac{19}{19}$ At both colliders, the dominant production mode is the gluon-fusion process. At the LHC, the vector boson fusion has the second largest cross section. In the low mass region it amounts at leading order to about $20 \%$ of the gluon-fusion cross section, whereas it reaches the same level for masses around $800 \mathrm{GeV} / \mathrm{c}^{2}$. At the Tevatron $p \bar{p}$ collider, the contribution of the associated $\mathrm{W} / \mathrm{Z} \mathrm{H}$ production mode is more important and, unlike at the LHC, Higgs boson searches heavily exploit this production mode. At the LHC, the associated $W H, Z H$ and $t \bar{t} H$ production processes are relevant only for the search of a light Standard Model Higgs boson with a mass close to the LEP limit.

For all production processes higher order QCD corrections have been calculated. In particular, significant progress has been made during the last two to three years in the calculation of QCD corrections for the gluon fusion and for the associated $t \bar{t} H$ and $b \bar{b} H$ production processes.

Already more than ten years ago, the next-to-leading order (NLO) QCD corrections to the gluon-fusion process have been calculated and have been found to be large.20 Their calculation appears challenging since already in the leading order diagram a massive one-loop triangle appears. The NLO calculation showed a significant increase of the predicted total cross section by about $50-100 \%$. These large corrections stimulated the calculation of the next-to-next-to-leading order (NNLO) corrections, to which many authors contributed 21222324 and which meanwhile has been completed in the heavy top-quark limit $\left(m_{t} \rightarrow \infty\right)$. In this limit the calculation of the Feynman 

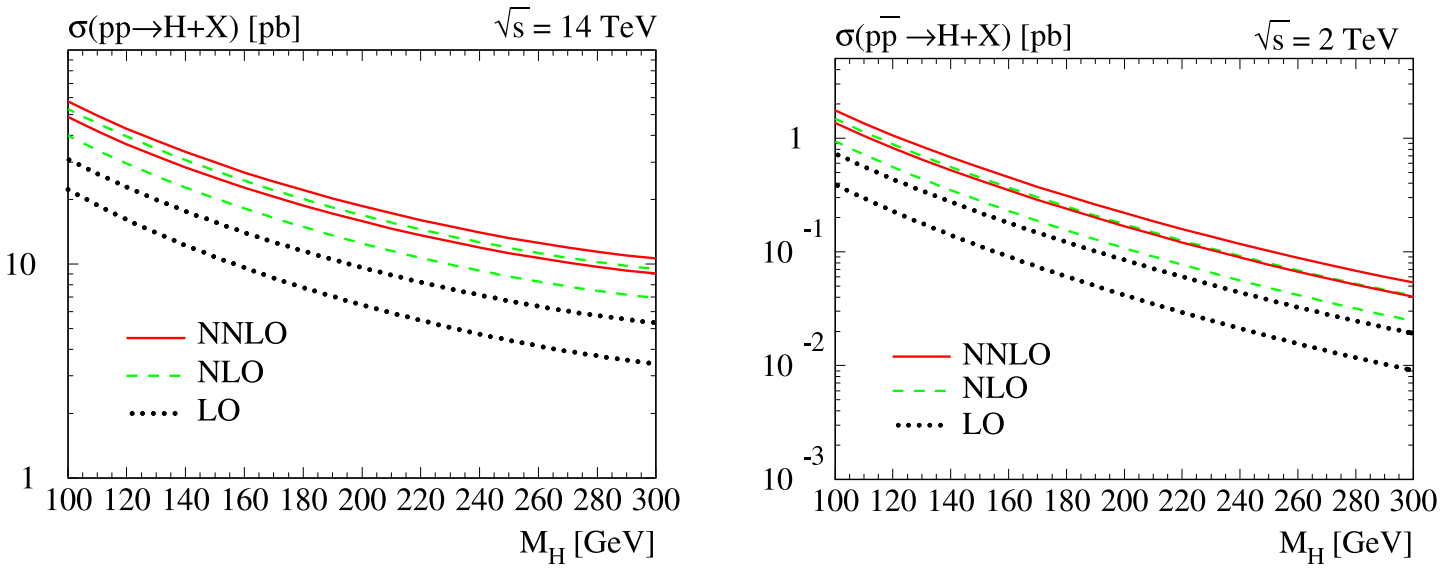

Figure 2: Gluon-fusion production cross section for a Standard Model Higgs boson at the LHC 14 TeV pp collider (left) and at the Tevatron $2 \mathrm{TeV} p \bar{p}$ collider (right) at leading (dotted), next-to-leading (dashed) and next-to-nextto-leading order (solid). The upper (lower) curve of each pair corresponds to a choice of the renormalization and factorization scale of $\mu_{R}=\mu_{F}=m_{H} / 2\left(\mu_{R}=\mu_{F}=2 m_{H}\right)$ (from Ref. [26 see also Refs. [22] [23] 24).

diagrams is considerably simplified. The approximation has been tested at NLO and has been found to agree within $5 \%$ with the full NLO calculation up to $m_{H}=2 m_{t}$, if the NLO result obtained with $\left(m_{t} \rightarrow \infty\right)$ is rescaled with the ratio of the LO cross sections calculated with a finite top mass and with $\left(m_{t} \rightarrow \infty\right)$. For larger Higgs boson masses still a surprisingly good agreement is found, e.g., the approximation deviates from the exact result by only $10 \%$ at $m_{H}=1 \mathrm{TeV} / \mathrm{c}^{2}$, if the same rescaling is applied ${ }^{25]}$ Based on these observations, it appears reasonable to apply the heavy topquark approximation also at NNLO. The results for the total cross section show a modest increase between the NLO and NNLO calculation at the level of 10-20\%, indicating that a nicely converging perturbative series seems to be emerging. The results of the LO, NLO and NNLO cross section calculations are shown for both colliders in Fig. 2. In particular, a clear reduction of the uncertainty due to higher order corrections has been observed, which is estimated to be about $15 \%$ based on variations of the renormalization scale. 25

Another important and challenging theoretical calculation constitutes the NLO calculation of the QCD corrections to the associated $t \bar{t} H$ production. Results have been published recently for a finite top-quark mass 2728 Also in this case a dramatic reduction in the variation of the cross-section prediction with the renormalization and factorization scale $\mu$ has been found 2728 as can be seen in Fig. [3] where the LO and NLO cross sections are shown as a function of the scale for both the LHC and the Tevatron. For the choice $\mu=m_{t}+m_{H} / 2$, for example, the NLO correction is found to be negative for the Tevatron, whereas a small increase of the cross section of the order of $20 \%$ is found for the LHC.

In the Standard Model, the cross section for producing a Higgs boson in association with $b$ quarks is relatively small. However, in a supersymmetric theory with a large value of $\tan \beta$, the $b$-quark Yukawa coupling can be strongly enhanced and Higgs boson production in association with $b$ quarks becomes the dominant production mechanism. Cross-section calculations including next-to-leading order corrections have been presented in two different approaches. 29 In the so-called four-flavour scheme, no $b$ quarks are present in the initial state and the lowest order processes are the $g g \rightarrow b \bar{b} h$ and $q \bar{q} \rightarrow b \bar{b} h$ tree level processes. Due to the splitting of gluons into $b \bar{b}$ pairs and the small $b$ quark mass, the inclusive cross section is affected by large logarithms and the convergence of the perturbative expansion may be poor. Depending on the final state considered, the convergence can be improved by summing the logarithms to all orders in perturbation theory through the use of $b$ quark parton distributions, i.e., moving to a five-flavour scheme. ${ }^{[0]}$ In this scheme, the lowest order inclusive process is $b \bar{b} \rightarrow h$. The first order correction to this process includes the process $g b \rightarrow b h$.

In many analyses two high- $P_{T} b$ quarks are required experimentally to improve the signal-tobackground ratio. The leading subprocess in this region of phase space is $g g \rightarrow b \bar{b} h$. The relevant production cross section, implementing parton level cuts on the $b$ quarks that closely reproduce the experimental cuts, have been computed at NLO in Refs. 31 and 32 for both the Tevatron and the 

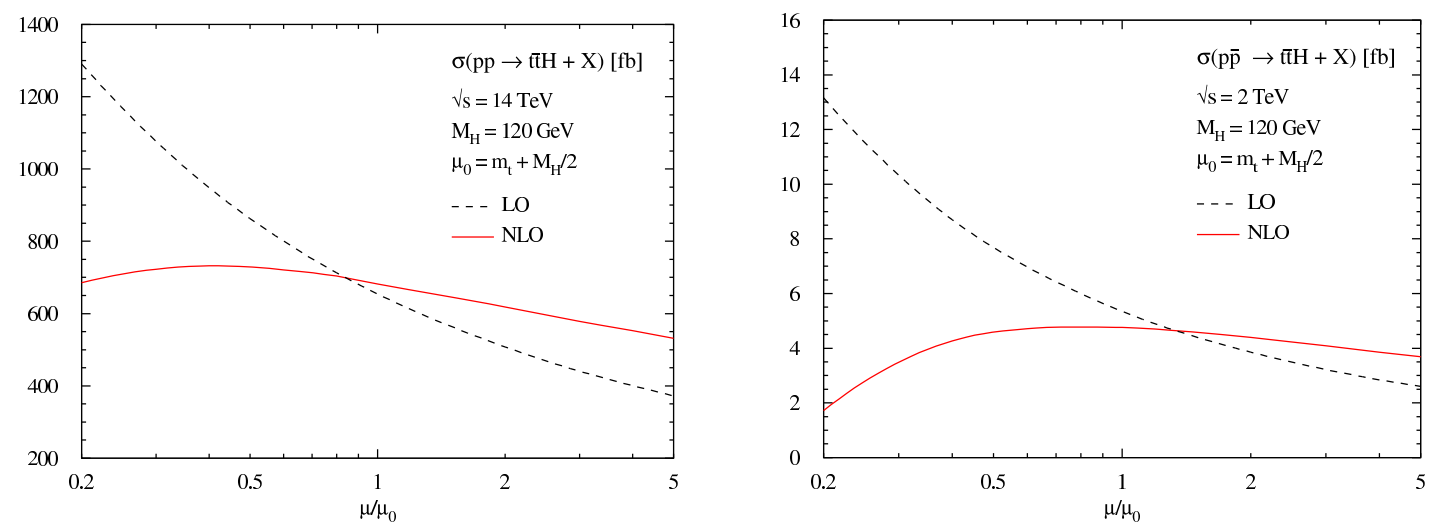

Figure 3: Variation of the $t \bar{t} H$ production cross section at the LHC $14 \mathrm{TeV}$ pp collider (left) and at the Tevatron $2 \mathrm{TeV} p \bar{p}$ collider (right) with the renormalization and factorization scale $\mu=\mu_{R}=\mu_{F}$, varied around the value $\mu_{0}=m_{t}+m_{H} / 2$ (from Refs. 27] and [28).

LHC. The NLO corrections modify the leading order predictions by less than $30 \%$ at the Tevatron and less than $50 \%$ at the LHC. For a cut of $P_{T}>20 \mathrm{GeV} / \mathrm{c}$ and $|\eta|<2.0(2.5)$ for the $b$ quarks at the Tevatron (LHC) and using $\mu=\left(2 m_{b}+m_{h}\right) / 4$ as factorization and renormalization scale, the NLO corrections are negative for small Higgs boson masses around $120 \mathrm{GeV} / \mathrm{c}^{2}$ and positive for large masses, with cross-over points around $140 \mathrm{GeV} / \mathrm{c}^{2}$ at the Tevatron and around $300 \mathrm{GeV} / \mathrm{c}^{2}$ at the LHC.

For cases of one and no tagged b-jets in the final state, results for the relevant cross sections can be calculated in the four- and five-flavour schemes. The two calculations represent different perturbative expansions of the same physics process and should agree at sufficiently high order. The NLO four-flavour result is obtained by integration over one of the $b$ quarks in the $g g \rightarrow b \bar{b} h$ calculation $[3132$ For the one-jet case, this calculation is found to agree within their respective scale uncertainties with the NLO calculation performed in the five-flavour scheme 33 For the inclusive cross section (no tagged $b$ jets) the five-flavour calculation has been performed to NLO in Ref. 34 and to NNLO in Ref. 35] Again, within the large respective uncertainties, agreement between the NLO four- and NNLO five-flavour calculation is found for small Higgs boson masses, while for large Higgs boson masses the five-flavour scheme tends to yield larger cross sections. This represents major progress compared to several years ago, when large discrepancies between the NLO $b b \rightarrow h$ and the LO $g g \rightarrow b \bar{b} h$ calculation had been reported $36: 37$

Next-to-leading order calculations of the production cross sections are also available for the remaining two production mechanisms: $W H, Z H$ and $q q H$ production. For the vector boson fusion process the NLO corrections are found to be moderate, 3839 i.e., at the level of $10 \%$. The NLO QCD corrections for the associated production of a Higgs boson with a vector boson can be derived from the Drell-Yan process and give a $30 \%$ increase with respect to the leading order prediction. 40

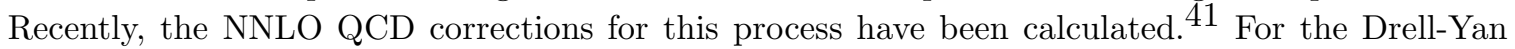
type corrections, a moderate increase of the NLO cross sections of up to $3 \%(10 \%)$ is found for the LHC (Tevatron) for a Higgs boson mass in the range $100<m_{H}<300 \mathrm{GeV} / \mathrm{c}^{2}$. For the $Z H$ associated production, additional gluon fusion contributions appear at NNLO (a triangular diagram $g g \rightarrow Z^{*} \rightarrow Z H$ and a box diagram where the $\mathrm{Z}$ and $\mathrm{H}$ bosons are emitted from internal quark lines). These contributions have been found to be relevant only at the LHC and increase the $Z H$ production cross section in the low mass region by about $10 \%$.

The production cross sections for the four different processes including the NLO QCD corrections are shown in Fig. 4 for both the Tevatron and the LHC collider as a function of the Higgs boson mass 19

\subsection{Higgs boson decays}

The branching fractions of the Standard Model Higgs boson are shown in Fig. [5(left) as a function of the Higgs boson mass. They have been calculated taking into account both electroweak and QCD 

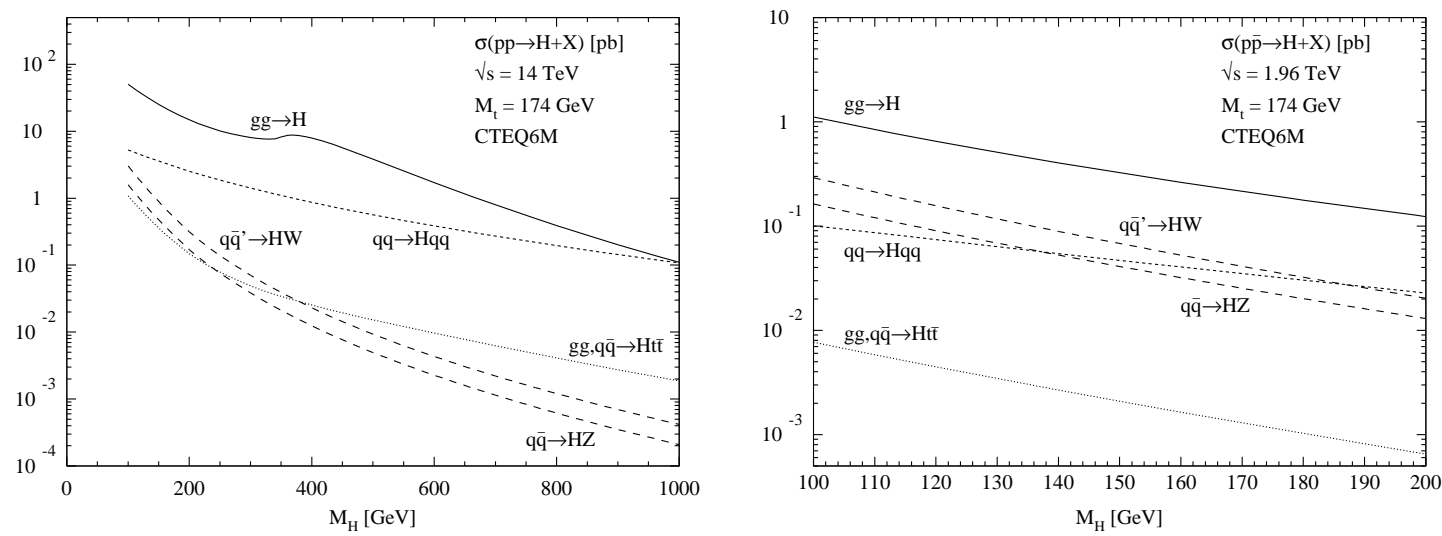

Figure 4: NLO production cross sections for a Standard Model Higgs boson as a function of the Higgs boson mass at

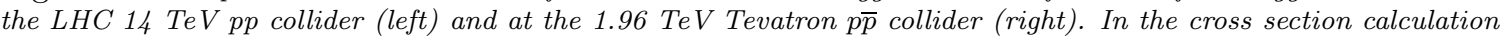
the CTEQ6M structure function parametrization has been used. (The calculations have been performed by M. Spira, Ref. 19)
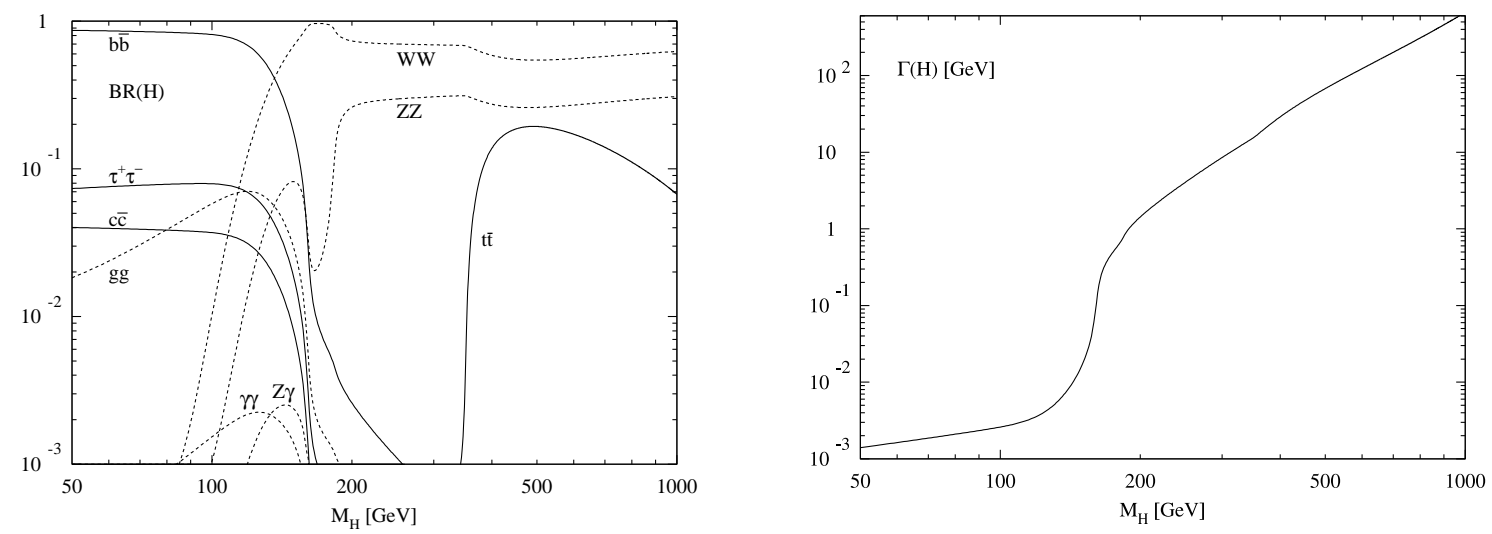

Figure 5: Branching fractions (left) and total decay width (right) of the Standard Model Higgs boson as a function of Higgs boson mass (from Ref. 42). 

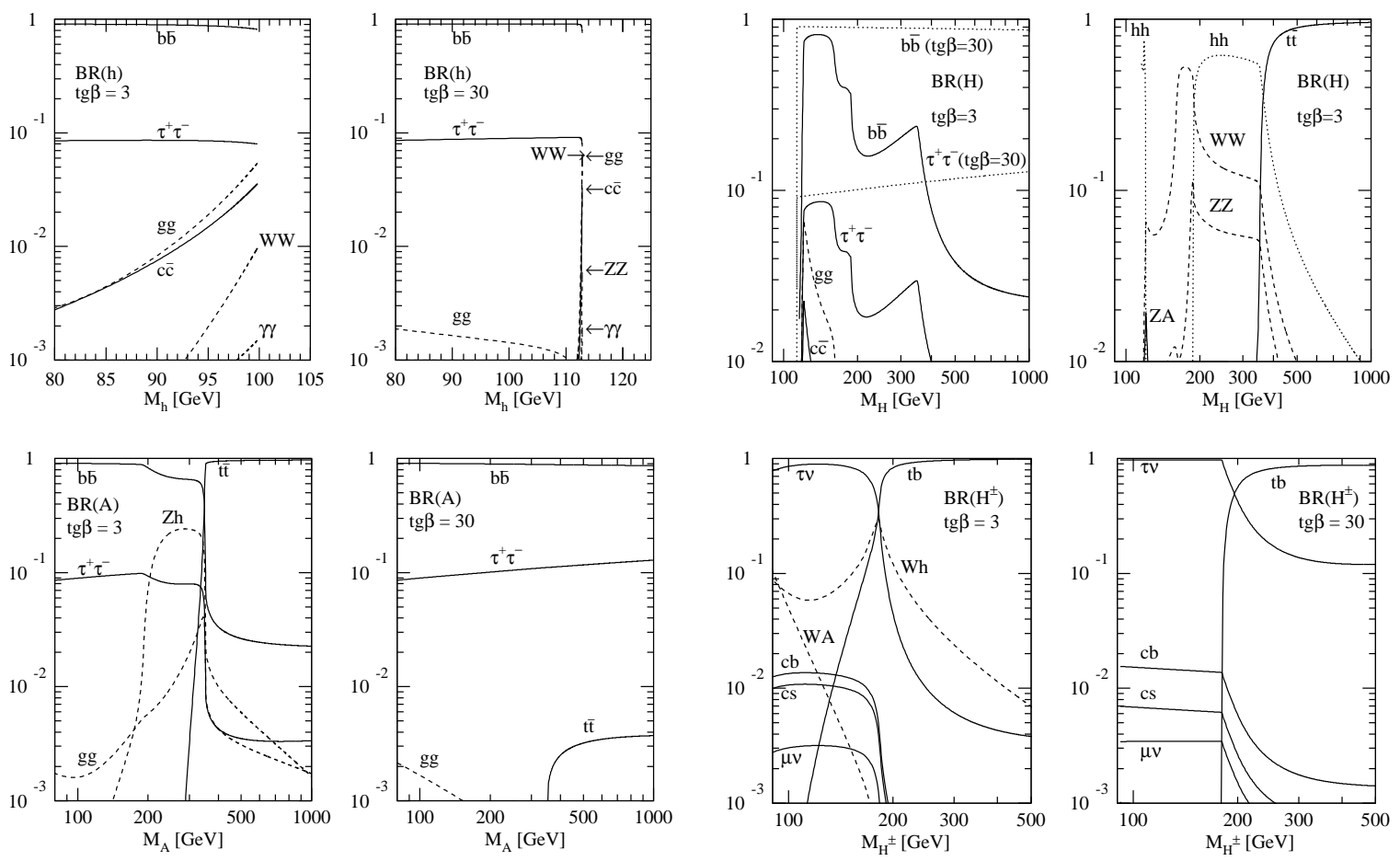

Figure 6: Branching fractions of the MSSM Higgs bosons as a function of their masses for tan $\beta=3$ and $\tan \beta=30$ assuming a SUSY mass scale of $1 \mathrm{TeV} / \mathrm{c}^{2}$ and vanishing mixing (from Ref. 42).

corrections 42 The latter include logarithmic corrections which are sizeable in particular for decays into $b$ or $c$ quarks. Large logarithms are resummed by using both the running quark mass and the strong coupling constant evaluated at the scale of the Higgs boson mass. When kinematically accessible, decays of the Standard Model Higgs boson into vector boson pairs $W W$ or $Z Z$ dominate over all other decay modes. Above the kinematic threshold, the branching fraction into $t \bar{t}$ can reach up to $20 \%$. All other fermionic decays are only relevant for Higgs boson masses below $2 m_{W}$, with $H \rightarrow b \bar{b}$ dominating below $140 \mathrm{GeV} / \mathrm{c}^{2}$. The branching fractions for $H \rightarrow \tau \tau$ and $H \rightarrow g g$ both reach up to about $8 \%$ at Higgs boson masses between 100 and $120 \mathrm{GeV} / \mathrm{c}^{2}$. Decays into two photons, which are of interest due to their relatively clean experimental signature, can proceed via fermion and $W$ loops with a branching fraction of up to $2 \cdot 10^{-3}$ at low Higgs boson masses.

Compared to the mass resolution of hadron collider experiments, the total decay width of the Standard Model Higgs boson is negligible at low masses and becomes significant only above the threshold for decays into $Z Z$, as shown in Fig. 5 (right). At $m_{H}=1 \mathrm{TeV} / \mathrm{c}^{2}$, the Higgs resonance is broad with a width of about $600 \mathrm{GeV} / \mathrm{c}^{2}$. In this mass regime, the Higgs field is coupling strongly, resulting in large NNLO corrections. With increasing coupling, the peak position of the Higgs boson resonance does not increase beyond a saturation value close to $1 \mathrm{TeV} / \mathrm{c}^{2}$, as found in both perturbative and non-perturbative calculations $\underline{43}$

Within the MSSM, branching fractions of five physical Higgs bosons have to be considered as a function of their masses as well as $\tan \beta$ and the masses of the SUSY particles. In Fig. 6 the branching fractions of all MSSM Higgs bosons are shown assuming that supersymmetric particles are heavy enough to be neglected. $\stackrel{42}{ }$ The neutral Higgs bosons decay dominantly into $b \bar{b}$ and $\tau^{+} \tau^{-}$ at large $\tan \beta$ or for masses below $150 \mathrm{GeV} / \mathrm{c}^{2}$ (up to $2 m_{t}$ for the CP-odd Higgs boson A). Decays into $W W, Z Z$ and photons are generally suppressed by kinematics as well as the Higgs couplings and become relevant only in the decoupling limit $m_{A} \rightarrow \infty$, where the light CP-even Higgs boson $h$ effectively behaves like a Standard Model Higgs boson while all other MSSM Higgs bosons are heavy. Charged Higgs bosons preferably decay into $t b$ if accessible. For masses below $m_{t}+m_{b}$, the decay $H^{ \pm} \rightarrow \tau \nu$ dominates, with small contributions from $H^{ \pm} \rightarrow c b$ and $H^{ \pm} \rightarrow c s$. In addition, both charged and heavy neutral Higgs bosons can decay into lighter Higgs bosons: $H^{ \pm} \rightarrow W h, W A$ as well as $H \rightarrow h h, A A, Z A$ and $A \rightarrow Z h$. Generally, the branching fractions of these decay modes are 
significant only at small $\tan \beta$.

Supersymmetric particles can influence the phenomenology of Higgs decays either via loop effects or, if light enough, as viable decay modes. In particular, Higgs boson decays to charginos, neutralinos and third generation sfermions can be relevant if kinematically allowed. With R-parity conserved, Higgs boson decays into the lightest neutralino are not directly detectable ("invisible decay").

\section{Experimental Scenarios}

\subsection{The Tevatron Run II}

After a successful first period of data taking until 1996 (Run I), the Fermilab accelerator complex has been upgraded to provide collisions with increased luminosity and a centre-of-mass energy of $1.96 \mathrm{TeV}$. In 2001 the second phase (Run II) of the experimental program started. After an initial period with low luminosity, peak luminosities of up to $1.0 \cdot 10^{32} \mathrm{~cm}^{-2} \mathrm{~s}^{-1}$ have been reached in Summer 2004. A further significant increase in luminosity is expected after the commissioning of the recycler, a new ring that will be used for accumulation and cooling of antiprotons. With this, the accelerator is expected to deliver an integrated luminosity of up to $8 \mathrm{fb}^{-1}$ by 2009 .

Both Tevatron experiments CDF and $\mathrm{D} \varnothing$ have undergone major upgrades to meet the requirements of the Run II physics program as well as the higher luminosity and collision rates of the upgraded accelerator. In the design, particular emphasis has been placed on achieving an efficient identification of leptons and b-jets as well as on providing good jet and missing energy measurements. The CDF and DØ detectors are described in detail in Refs. 44 and 45. Only a brief overview is presented in the following.

The CDF tracking system consists of silicon detectors and a drift chamber situated inside a solenoid that provides a $1.4 \mathrm{~T}$ magnetic field coaxial with the beam. The silicon microstrip detector has eight cylindrical layers of mostly double-sided silicon, distributed in radius between $1.5 \mathrm{~cm}$ and $28 \mathrm{~cm}$. The system is read out in about 700.000 channels and can provide three-dimensional precision tracking up to pseudorapidities of 2.0. Outside of the silicon detectors and for pseudorapidities less than 1.0, charged particles are detected with up to 96 hits per track by the central outer tracker, an open-cell drift chamber with alternating axial and $2^{\circ}$ stereo superlayers with 12 wires each. Just inside the solenoid, a scintillator-based time-of-flight detector allows particle identification with a timing resolution of about 100 ps.

The electromagnetic (hadronic) calorimeters are lead-scintillator (iron-scintillator) sampling calorimeters, providing coverage up to pseudorapidities of 3.6 in a segmented projective tower geometry. Proportional wire and scintillating strip detectors situated at a depth corresponding to the electromagnetic shower maximum provide measurements of the transverse shower profile. In addition, an early energy sampling is obtained using preradiator chambers positioned between the solenoid coil and the inner face of the central calorimeter. Outside of the calorimeter and behind additional steel absorbers, a multi-layer system of drift chambers and scintillation counters allows detection of muons for pseudorapidities up to 1.5 .

The tracking system of the $\mathrm{D} \varnothing$ detector consists of a silicon vertex detector and a scintillating fibre tracker, situated inside a superconducting coil providing a $2 \mathrm{~T}$ magnetic field. The D $\varnothing$ silicon tracker has four cylindrical layers of mostly double-sided microstrip detectors covering $2.7 \mathrm{~cm}$ up to $9.4 \mathrm{~cm}$ in radius, interspersed with twelve disk detectors in the central region and two large disks in either forward region. The full system has about 800.000 channels and provides three-dimensional precision tracking up to pseudorapidities of 3.0. The volume between the silicon tracker and the superconducting coil is instrumented with eight cylindrical double layers of scintillating fibres. Each layer has axial and stereo fibres (stereo angle $\pm 3^{\circ}$ ) with a diameter of $835 \mu \mathrm{m}$, that are read out using solid-state photodetectors (Visible Light Photon Counters, VLPCs).

The $\mathrm{D} \varnothing$ calorimeter is a Liquid Argon sampling calorimeter with uranium absorber (copper and steel for the outer hadronic layers) with hermetic coverage up to pseudorapidities of 4.2. Signals are read out in cells of projective towers with four electromagnetic, at least four hadronic layers and a transverse segmentation of 0.1 in both azimuth and pseudorapidity. The granularity is increased to 0.05 for the third EM layer, roughly corresponding to the electromagnetic shower maximum. To provide additional sampling of energy lost in dead material, scintillator-based detectors are placed in front of the calorimeter cryostats (preshower detectors) and between the barrel and end-cap 
cryostats (intercryostat detector). The preshower detectors consist of three layers of scintillator strips with VLPC readout providing, in addition to the energy measurement, a precise three-dimensional position measurement for electromagnetic showers.

The D $\varnothing$ muon system consists of three layers of drift tubes and scintillators, with toroid magnets situated between the first and second layer to allow for a stand-alone muon momentum measurement. Scintillator pixels are used for triggering and rejection of out-of-time backgrounds in both the central and forward regions. Proportional drift tubes are stacked in three or four decks per layer in the central region. Tracking of muons in the forward region is accomplished by using decks of mini drift tubes in each layer, allowing muons to be reconstructed up to pseudorapidities of 2.0. The muon system is protected from beam-related backgrounds by shielding around the beampipe using an iron-polyethylene-lead absorber.

Both CDF and DØ detectors are read out using a three-level trigger system which reduces the event rate from $2.5 \mathrm{MHz}$ to about $50 \mathrm{~Hz}$. This includes programmable hardware triggers at Level 1 that provide basic track, lepton and jet reconstruction, secondary vertex or impact parameter triggers at Level 2 as well as a PC-based quasi-offline event reconstruction at Level 3.

After commissioning, calibration and alignment, about $500 \mathrm{pb}^{-1}$ of physics quality data have been collected by each experiment between April 2002 and July 2004. Physics results based on the analysis of up to $200 \mathrm{pb}^{-1}$ have been presented at the Summer Conferences 2004.

\subsection{LHC experiments}

The Large Hadron Collider ( $L H C$ ) is presently being constructed as a proton-proton collider with a centre-of-mass energy of $14 \mathrm{TeV}$ at CERN. This machine will open up the possibility to explore the $\mathrm{TeV}$ energy range, which plays a key role in the investigation of the electroweak symmetry breaking. Two experiments, ATLAS and CMS, have been designed and optimized as general purpose $p p$ detectors, capable of running at high luminosity $\left(\mathcal{L}=10^{34} \mathrm{~cm}^{-2} \mathrm{~s}^{-1}\right)$ and detecting a variety of final-state signatures. For details on the detector concepts, the reader is referred to the Technical

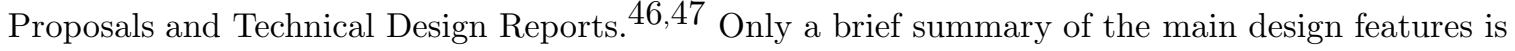
given in the following.

Both experiments use a superconducting solenoid around the inner detector cavity to measure the track momenta. Pattern recognition, momentum and vertex measurements are achieved with a combination of high-resolution silicon pixel and strip detectors. In the ATLAS experiment, tracking is performed in a $2 \mathrm{~T}$ magnetic field. Electron identification is enhanced with a continuous strawtube tracking detector with transition radiation capability in the outer part of the tracking volume. Due to the presence of silicon strip and pixel detectors, both experiments will be able to perform $b$ quark tagging using impact parameter measurements and the reconstruction of secondary vertices.

The calorimeter of the ATLAS experiment consists of an inner barrel cylinder and end-caps, using the intrinsically radiation resistant Liquid Argon (LAr) technology. Over the full length, this calorimeter is surrounded by a novel hadronic calorimeter using iron as absorber and scintillating tiles as active material. The barrel part of the LAr calorimetry is an electromagnetic accordion calorimeter, $\frac{46}{16}$ with a highly granular first sampling (integrated preshower detector). In the endcap region $(1.5<|\eta|<3.2)$ the LAr technology is used for both electromagnetic and hadronic calorimeters. In order to achieve a good jet and missing transverse energy $\left(\mathbb{E}_{T}\right)$ measurement, the calorimeter coverage is extended down to $|\eta|<4.9$ using a special forward LAr calorimeter with rod-shaped electrodes in a tungsten matrix. The CMS calorimeter system $\frac{47}{\text { consists of a }}$ high resolution lead tungstate $\left(\mathrm{PbWO}_{4}\right)$ crystal calorimeter, complemented by a hadronic copperscintillator sandwich calorimeter. Both the electromagnetic and a large fraction of the hadronic calorimeter are located inside the coil of the solenoid. The choice of the detection technique is to a large extent motivated by the search for the Higgs boson in the $H \rightarrow \gamma \gamma$ decay channel, which requires both an excellent electromagnetic energy and angular resolution.

In addition to the inner solenoid, the ATLAS detector has large superconducting air-core toroids consisting of independent coils arranged outside the calorimetry. This allows a stand-alone muon momentum measurement with three stations of high-precision tracking chambers at the inner and outer radius and in the middle of the air-core toroid. In the CMS experiment the magnetic flux of the large solenoid is returned through a $1.8 \mathrm{~m}$ thick saturated iron return yoke $(1.8 \mathrm{~T})$ which is instrumented with muon chambers. A single magnet thus provides the necessary bending power 
for precise tracking in the inner detector and in the muon spectrometer. The magnetic field in the central cavity, in which the inner detectors are located, is $4 \mathrm{~T}$.

It is assumed that at the LHC an initial luminosity of $10^{33} \mathrm{~cm}^{-2} \mathrm{~s}^{-1}$, hereafter called low luminosity, can be achieved at the startup, which is expected for the year 2007. This value is supposed to increase during the first two to three years of operation to the design luminosity of $10^{34}$ $\mathrm{cm}^{-2} \mathrm{~s}^{-1}$, hereafter called high luminosity. Integrated luminosities of $10 \mathrm{fb}^{-1}$ and $100 \mathrm{fb}^{-1}$ should therefore be collected at the LHC after about one and four years of data taking, respectively.

\section{Search for Higgs Bosons at the Tevatron}

As the Run II luminosity increases, the Tevatron experiments CDF and D $\varnothing$ will start reaching sensitivity to production of low-mass Higgs bosons beyond the LEP limits. For Standard Model Higgs bosons decaying to $b \bar{b}$, the production in association with $W$ or $Z$ bosons is the most promising channel. In the mass range between $\sim 150$ and $\sim 180 \mathrm{GeV} / \mathrm{c}^{2}$, Higgs bosons produced via gluon fusion might be observable in their decays to $W W$. Given the current projections for the integrated Tevatron luminosity of about $8 \mathrm{fb}^{-1}$ by 2009 , a $5 \sigma$ discovery of a Standard Model Higgs boson will be very difficult to achieve. Nevertheless, combining results from all search channels should provide sensitivity for exclusion of Higgs boson production at the $95 \%$ confidence level up to Higgs boson masses of $180 \mathrm{GeV} / \mathrm{c}^{2}$.

In the following subsections the current status and projections for the most important channels at Tevatron Run II are summarized. When available, calculations of signal and background cross sections beyond leading order have been used. Systematic errors are taken into account unless specifically noted otherwise. Over the last couple of years, the original Monte Carlo studies presented in Ref. 36 have been cross-checked and refined using Run II data and hit-based GEANT 48 simulation. $\frac{49}{9}$ In most channels, first preliminary results from the analysis of about $200 \mathrm{pb}^{-1}$ of data exist and are summarized in the following.

In view of the reduced luminosity expectations for Run II, challenging search channels, such as $t \bar{t} H$ and diffractive Higgs production,, 5036 are expected to provide little sensitivity and will therefore not be discussed further.

\subsection{Associated production}

The production of Higgs bosons in association with vector bosons can be searched for in all leptonic decays of $W$ and $Z: W \rightarrow \ell \nu, Z \rightarrow \nu \bar{\nu}$ and $Z \rightarrow \ell \ell$ (with $\ell=e, \mu, \tau$ ). Sensitivity studies based on Monte Carlo simulation of the detector performance throughout the course of Run II exist 3649 For $W H$ production, these studies are compared to first preliminary results of searches in Run II data corresponding to integrated luminosities of $162 \mathrm{pb}^{-1}(\mathrm{CDF})$ and $174 \mathrm{pb}^{-1}(\mathrm{D} \varnothing)$ ! 51

Final states compatible with the $W H / Z H$ signature can be selected by requiring leptons and/or missing $E_{T}$ as well as two b-tagged jets. After offline cuts, the trigger efficiencies for events involving charged leptons are very close to $100 \%$, as measured in Run II data. The channel $Z H \rightarrow \nu \bar{\nu} b \bar{b}$ represents a challenge for the trigger systems of both experiments because of high rates due to the QCD background. Nevertheless, using a set of inclusive triggers exploiting calorimeter information at Level 1 combined with jet-, lepton- and impact-parameter-triggers at Level 2, DØ estimates a trigger efficiency of $>90 \%$ for events surviving offline analysis cuts.49

Backgrounds involving light-quark jets $(\mathrm{u}, \mathrm{d}, \mathrm{s})$ are suppressed using b-tagging, which for both experiments has an efficiency of about $50 \%$, as measured in data for central jets with $P_{T}>40 \mathrm{GeV} / c$

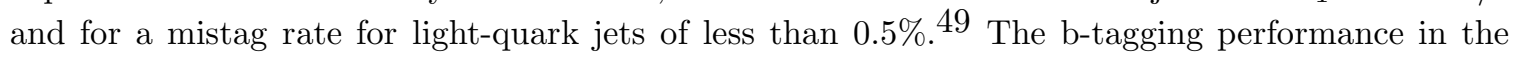
forward region has been estimated using Monte Carlo simulation; it is expected to improve further after including silicon stand-alone tracking algorithms using the forward silicon detectors.

After the b-tagging requirements and additional topological cuts, backgrounds in channels involving charged leptons are entirely dominated by physics backgrounds from $W b \bar{b}, Z b \bar{b}, W Z$ and $t \bar{t}$ production. For $Z H \rightarrow \nu \bar{\nu} b \bar{b}$, a significant amount of background from QCD jet production remains in addition. To improve the signal-to-background ratio further, the Higgs boson mass has to be reconstructed with the best possible resolution. Currently, a relative jet energy resolution of $13.9 \%$ has been achieved by DØ, as measured in Run II data for central jets at $E_{T}=55 \mathrm{GeV} \stackrel{49}{ }$ It is 

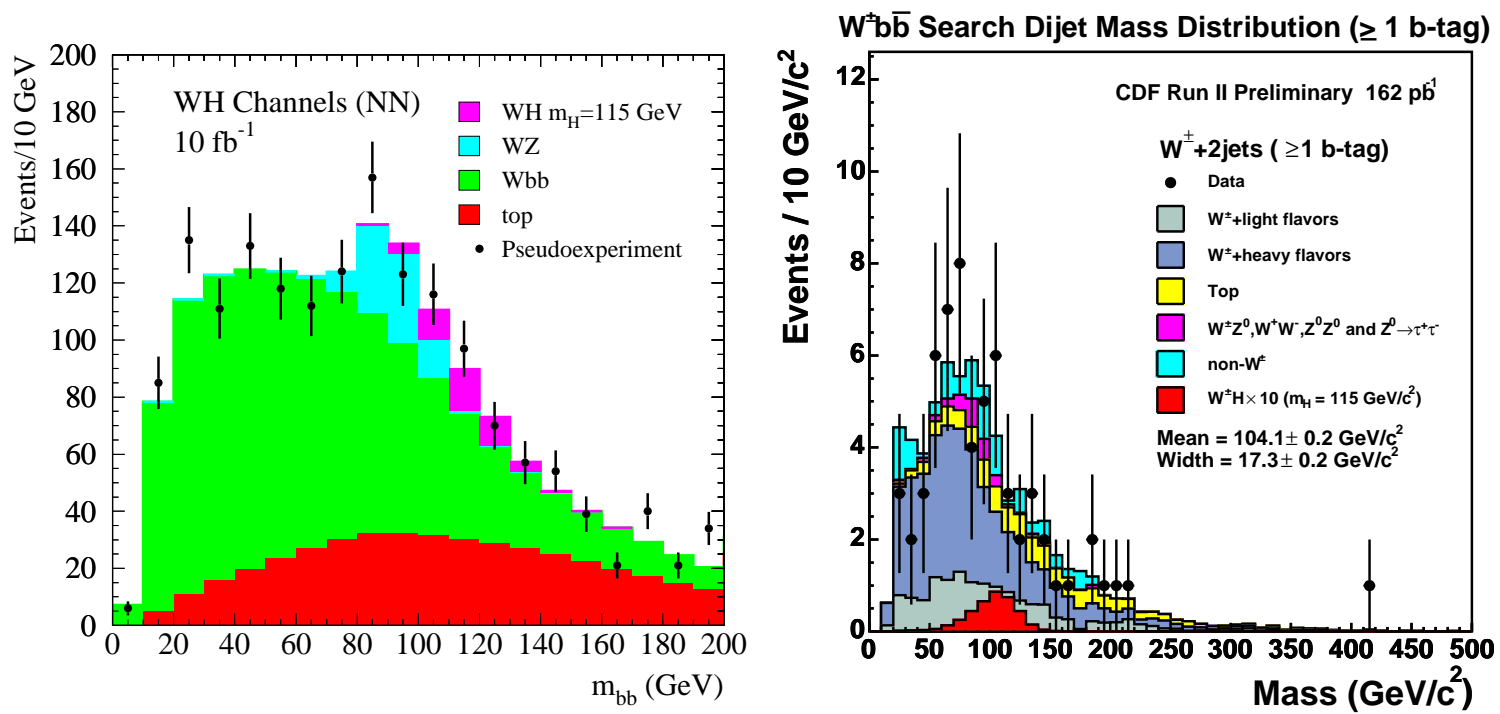

Figure 7: Invariant $b \bar{b}$ mass spectrum in search for WH production in full simulation of Tevatron data corresponding to an integrated luminosity of $10 \mathrm{fb}^{-1}$ (left, from Ref. 49) and in CDF Run II data corresponding to an integrated luminosity of $162 \mathrm{pb}^{-1}$ in comparison with signal and background expectation (right, from Ref. 51).

expected that this can be improved by $30 \%$ due to more sophisticated jet reconstruction algorithms as well as refinements in jet energy calibration, including a calibration of the $b \bar{b}$ mass reconstruction using the $Z \rightarrow b \bar{b}$ signal.

A Higgs signal is then searched for as an excess in the $b \bar{b}$ mass spectrum, as shown in Fig. Z(left) for full simulation of Tevatron data corresponding to an integrated luminosity of $10 \mathrm{fb}^{-1}$. Given the Higgs event yields ( $3 W H$ events selected per $\mathrm{fb}^{-1}$ ), this requires precise knowledge of the backgrounds over the entire mass range. While the normalization of the background can be obtained from a fit outside of the signal region, the shape and relative normalization of the $b \bar{b}$ mass spectrum of the various background components has to be known to allow extrapolation below the Higgs peak. Procedures to obtain this information from data are outlined in Ref. 49 and typically involve a measurement of the shape of the dijet mass spectrum in background-enriched samples, which is then extrapolated to the final signal sample using a mixture of Monte Carlo and data-driven methods.

The $b \bar{b}$ mass spectrum as measured in Run II data corresponding to an integrated luminosity of $162 \mathrm{pb}^{-1}$ is shown in Fig. 7(right) after all cuts. In this version of the analysis only one jet is required to be b-tagged. No evidence for $W H$ production is observed in current Run II searches by $\mathrm{CDF}$ and $\mathrm{D} \varnothing$, allowing to set an upper limit on the product of cross section and branching fraction $\sigma(W H) \times \mathrm{BR}(H \rightarrow b \bar{b})$ of $5 \mathrm{pb}$ for a Higgs boson mass of $120 \mathrm{GeV} / \mathrm{c}^{2} ! 51$ Due to the small amount of integrated luminosity that has been collected so far, the limit is still more than an order of magnitude higher than the Standard Model expectation. In Fig. 8 the luminosity required to observe (or exclude) a Standard Model Higgs boson is shown as a function of mass. This estimate assumes a 30\% improvement in jet energy resolution and anticipates a number of enhancements to the current analysis, including the use of forward b-tagging and multivariate methods as well as an increase in lepton acceptance by extending the analysis to the forward region and using isolated tracks to identify leptons. Systematic errors have not been taken into account. After combining all channels and both experiments, a sensitivity at the $95 \%$ C.L. for $\mathrm{m}_{H}=120 \mathrm{GeV} / \mathrm{c}^{2}$ is expected to be achieved with an integrated luminosity of $1.8 \mathrm{fb}^{-1}$ per experiment. Evidence for a signal at the $3 \sigma(5 \sigma)$ level will require $4 \mathrm{fb}^{-1}\left(10 \mathrm{fb}^{-1}\right)$ per experiment for the same Higgs boson mass.

\subsection{Search for $H \rightarrow W W$}

Higgs boson decays into two $W$ bosons are the dominant decay mode for masses above $140 \mathrm{GeV} / \mathrm{c}^{2}$. The relatively clean signature of two leptonic $W$ decays allows a search for this decay in the gluon- 


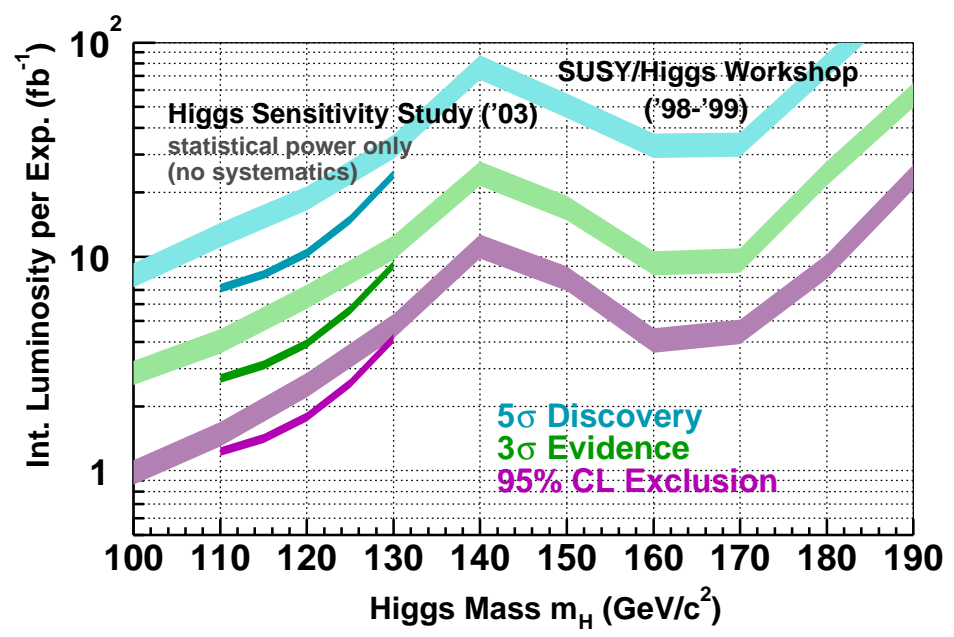

Figure 8: The integrated luminosity required per experiment for a 95\% C.L. exclusion, a $3 \sigma$ and a $5 \sigma$ discovery of a Standard Model Higgs boson at the Tevatron as a function of the Hiqqs boson mass. Thin lines show recent estimates based on full simulation verified with Run II datd 49 for the combination of searches for associated production with a vector boson with no systematic uncertainties included. Thick lines indicate results of an earlier study ${ }^{36}$ with fast simulation which includes the combination with searches for $g g \rightarrow H \rightarrow W W$ (see Section [4.2). For the latter, the line thickness indicates the impact of systematic uncertainties.

fusion channel. While the production cross section in this channel is higher compared to the associated production, the suppression due to the branching fractions of the leptonic $W$ decays limits the event yield to only about 4 events per $\mathrm{fb}^{-1}$ for $H \rightarrow W W \rightarrow e e, e \mu, \mu \mu+E_{T}$ with $m_{H}=160 \mathrm{GeV} / \mathrm{c}^{2}$.

Both Tevatron collaborations have started analyzing their Run II data in search for a $H \rightarrow W W$ signal. ${ }^{52}$ So far, efficiencies of up to $15-20 \%$ have been achieved for the dilepton plus $E_{T}$ final states with electrons or muons. The background is dominated by $W W$ production, which remains after selection cuts with a cross section of about $25 \mathrm{fb}$ for the sum of all three analysis channels. Further separation of signal and $W W$ events is possible using the difference in azimuthal angle $\Delta \phi$ between the two charged leptons. ${ }^{53}$ Due to spin correlations, $\Delta \phi$ tends to be small for decays of a spin-0 resonance (see Fig. 9] and Section 5.2). Both CDF and D $\varnothing$ observe no significant excess of events in Run II data corresponding to an integrated luminosity of $184 \mathrm{pb}^{-1}$ and $176 \mathrm{pb}^{-1}$, respectively. Limits on the production cross section of $H \rightarrow W W$ have been set as a function of the Higgs boson mass as shown in Fig. 10] For a mass of $160 \mathrm{GeV} / \mathrm{c}^{2}$, cross sections larger than 5.6 pb have been excluded at $95 \%$ C.L., which is still more than an order of magnitude higher than the expectation within the Standard Model.

The performance of these analyses is consistent with the expectations based on the fast simulation, which projected a total background of $30.4 \mathrm{fb}$ at an efficiency of $18.5 \%$ for a Higgs boson mass of $150 \mathrm{GeV} / \mathrm{c}^{2}$. 36 Based on this projection, sensitivity at the $95 \%$ C.L. to a Standard Model Higgs boson with masses between 160 and $170 \mathrm{GeV} / \mathrm{c}^{2}$ will be reached with an integrated luminosity of $4 \mathrm{fb}^{-1}$ per experiment (10 $\mathrm{fb}^{-1}$ for a $3 \sigma$ sensitivity), as shown in Fig. 8 These estimates include contributions from the vector boson fusion channel and Higgs boson production in association with vector bosons. The latter is best searched for using a like-sign dilepton selection. ${ }^{5436}$ First results in this channel have been reported by the CDF collaboration, but are not yet competitive with the gluon-fusion channel. 55

In models beyond the Standard Model, the rate of $H \rightarrow W W$ events can be enhanced due to larger production cross sections (models with heavy 4th generation quarks) or due to an increase in branching fraction (Topcolor models 56 ). In the former case, the gluon-fusion process is enhanced due to loop-diagrams involving heavy quarks by a factor of about 8.5 within the mass range of interest at the Tevatron, with only a mild dependence on the heavy quark mass .57 The latter class 

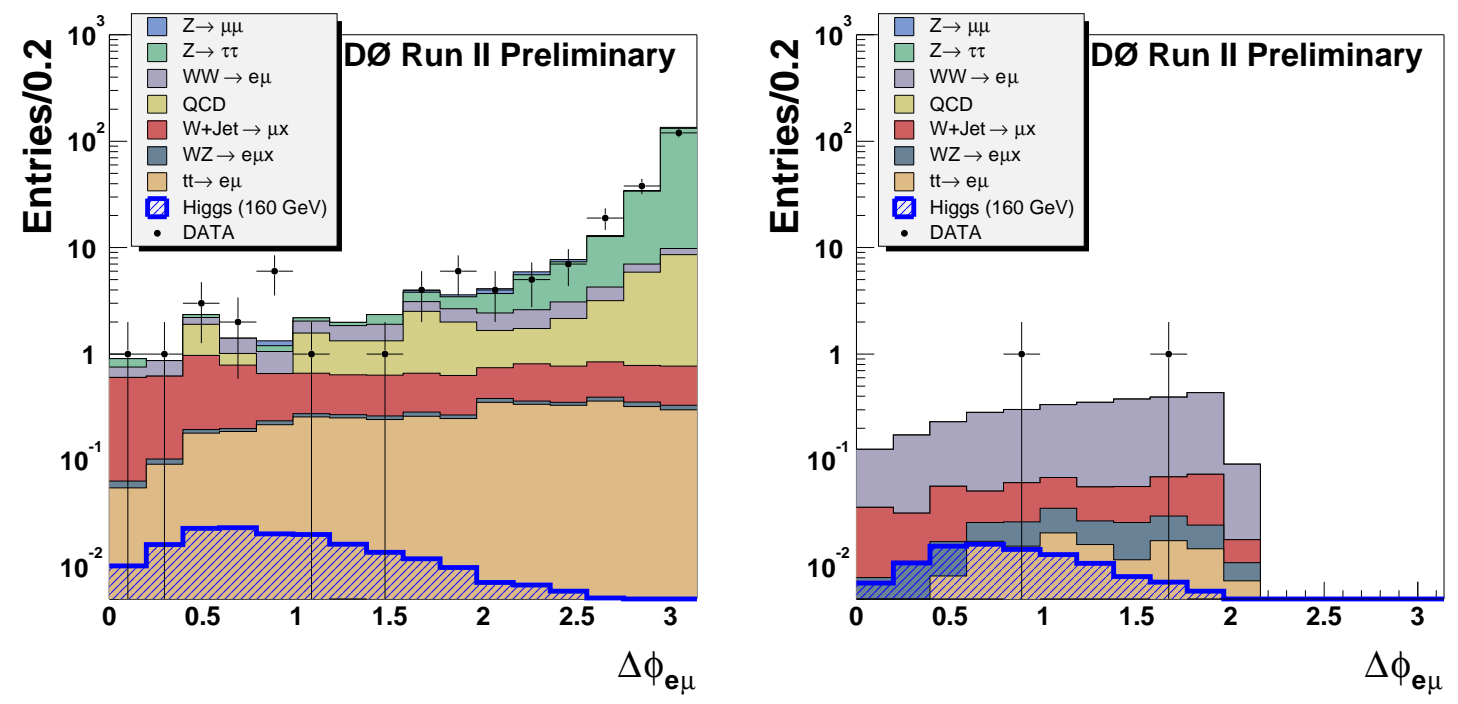

Figure 9: Difference in azimuthal angle of the reconstructed electron and muon at an early stage of the selection (left) and after all cuts (right) in the search for $H \rightarrow W W \rightarrow e \nu \mu \nu$ in DØ Run II data corresponding to an integrated luminosity of $176 \mathrm{pb}^{-1}$ (from Ref. 52).

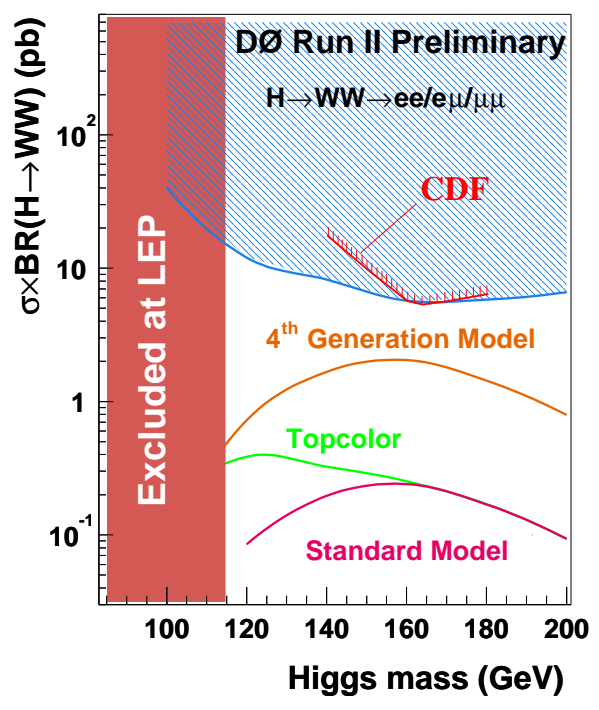

Figure 10: Upper limit (at 95\% C.L.) on the cross section $\sigma \cdot B R(H \rightarrow W W)$ set by the DØ Collaboration using Run II data corresponding to an integrated luminosity of $176 \mathrm{pb}^{-1}$, with the limit set by the CDF Collaboration (184 $\mathrm{pb}^{-1}$ ) superimposed (from Ref. 52). The limits are compared to expectations from Standard Model Higgs production and alternative models. 
of models also predicts an enhanced branching fraction for $H \rightarrow \gamma \gamma$, which can be searched for with

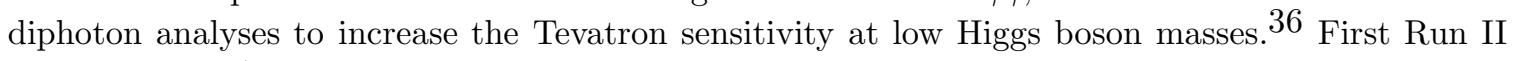
results from $\mathrm{D} \varnothing$ in this channel exist, but improve only marginally on existing limits from LEP and Run I.

\subsection{Neutral Higgs bosons in supersymmetry}

Sensitivity to a low-mass Higgs boson is of particular interest within supersymmetric extensions of the Standard Model, which predict the existence of at least one neutral Higgs boson $\Phi=h, H, A$ with a mass below $\sim 135 \mathrm{GeV} / \mathrm{c}^{2}$. Searches for the Standard Model Higgs boson produced in association with a vector boson (cf. Section 4.1) can be interpreted within SUSY parameter space. $\frac{36}{16}$ In addition, the enhancement of the Higgs coupling to $b \bar{b}$ at large $\tan \beta$ results in sizeable cross sections for two search channels that are inaccessible within the Standard Model: the production of Higgs bosons in association with one or more $b$ quarks 33 as well as the gluon-fusion channel $g g \rightarrow \Phi$ with the subsequent decay $\Phi \rightarrow \tau \tau$.

\subsection{1 $\Phi b(b) \rightarrow b b b(b)$}

The $\mathrm{D} \varnothing$ collaboration has analyzed a Run II dataset corresponding to an integrated luminosity of $131 \mathrm{pb}^{-1}$, collected with multijet triggers optimized for the $\Phi b \bar{b} \rightarrow b \bar{b} b \bar{b}$ signal. ${ }^{[59}$ Requiring two jets with transverse momenta $P_{T}>25 \mathrm{GeV} / c$ and a third jet with $P_{T}>15 \mathrm{GeV} / c$, this trigger consumed less than $4 \mathrm{~Hz}$ of Level-3 bandwidth at instantaneous luminosities of $4.0 \cdot 10^{31} \mathrm{~cm}^{-2} \mathrm{~s}^{-1}$, while maintaining a signal efficiency of about $70 \%$ after offline cuts. The offline analysis requires at least three b-tagged jets with $P_{T}>15 \mathrm{GeV} / c$. Depending on the Higgs mass hypothesis, the $P_{T}$ cuts for the two leading jets are tightened to values between 35 and $60 \mathrm{GeV} / c$ to optimize for best expected sensitivity.

The background at this stage is dominated by multijet production with $b$ quarks. The signal can be searched for as a peak in the invariant mass spectrum of the two leading jets, which is shown in Fig. 111 in comparison with the expectation from background and a Higgs signal with $\mathrm{m}_{\Phi}=120 \mathrm{GeV} / \mathrm{c}^{2}$. The shape of the dijet mass spectrum in background is obtained from a multijet sample with two b-tagged jets, which is expected to have negligible contamination from signal, by weighting events using b-tag fake rates measured in data as a function of jet $P_{T}$ and $\eta$. The background is then normalized by fitting this shape to the mass spectrum outside the signal region.

No evidence for production of neutral Higgs bosons $h, H, A$ in association with b-jets has been observed, and limits on the production cross section have been set at 95\% C.L. This limit has been translated into an exclusion region in the $\left(m_{A}, \tan \beta\right)$-plane under the assumption that the production cross section is proportional to $\tan ^{2} \beta$. However, it should be noted that in the large $\tan \beta$ region, higher order corrections to the bottom-Yukawa coupling are large. The resulting limit is shown in Fig. [1] in comparison with existing limits from LEP. The D $\varnothing$ Run II limit is significantly worse than the limit published in 2001 by the CDF collaboration, which was based on the analysis of Run I data corresponding to an integrated luminosity of $91 \mathrm{pb}^{-1} \underline{60}$ Detailed comparisons of both results indicate that the apparent loss of sensitivity observed by D $\varnothing$ can be traced back to the use of more recent cross section calculations and fits of parton distribution functions, which cause a significant reduction in signal acceptance and cross section compared to the CDF Run I analysis. 61

For an integrated luminosity of $5 \mathrm{fb}^{-1}$ and after combining results from both Tevatron experiments, the reach in $\tan \beta$ within the $m_{h}$-max scenario (see Section 7.4) will be extended down to about $\tan \beta=25$ for $\mathrm{m}_{A}=120 \mathrm{GeV} / \mathrm{c}^{2}$ at the $95 \%$ C.L., but deteriorates quickly with increasing $\mathrm{m}_{A}$.

\subsection{2 $\Phi \rightarrow \tau \tau$}

In addition to the dominant decay mode $\Phi \rightarrow b \bar{b}$, a light supersymmetric Higgs boson can be searched for in its decay to $\tau^{+} \tau^{-}$. This decay mode is of particular interest both for SUSY scenarios that favour suppressed couplings of Higgs bosons to $b$ quarks as well as for the large $\tan \beta$ region, where the channels $\Phi b(b) \rightarrow \tau \tau b(b)$ and $g g \rightarrow \Phi \rightarrow \tau \tau$ provide a viable complement to the search for $\Phi b(b) \rightarrow b b b(b)$. 

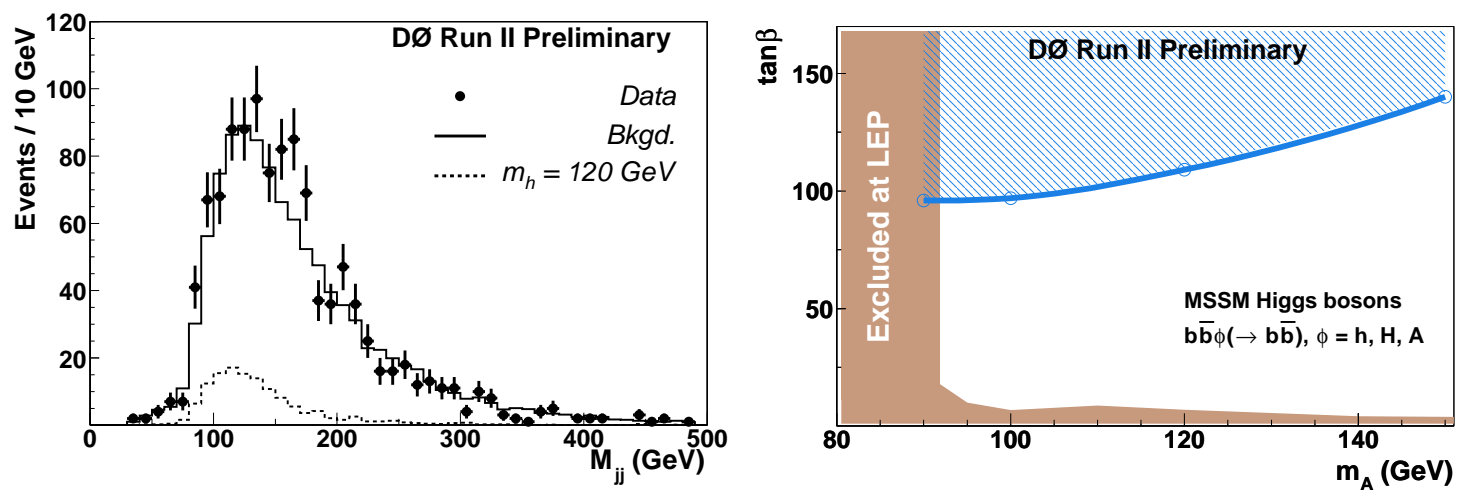

Figure 11: (Left) Invariant mass spectrum of the two leading jets in the 3-jet sample with three b-tags after final cuts in the DØ search for bh production using Run II data corresponding to an integrated luminosity of $131 \mathrm{pb}^{-1}$; the dashed histogram shows the distribution for a Higgs signal with $m_{H}=$ $120 \mathrm{GeV} / \mathrm{c}^{2}$ and $\tan \beta=100$; (Right) Regions in $\left(m_{A}, \tan \beta\right)$ excluded by this analysis at $95 \%$ C.L. in comparison with LEP limits (from Ref. [59).
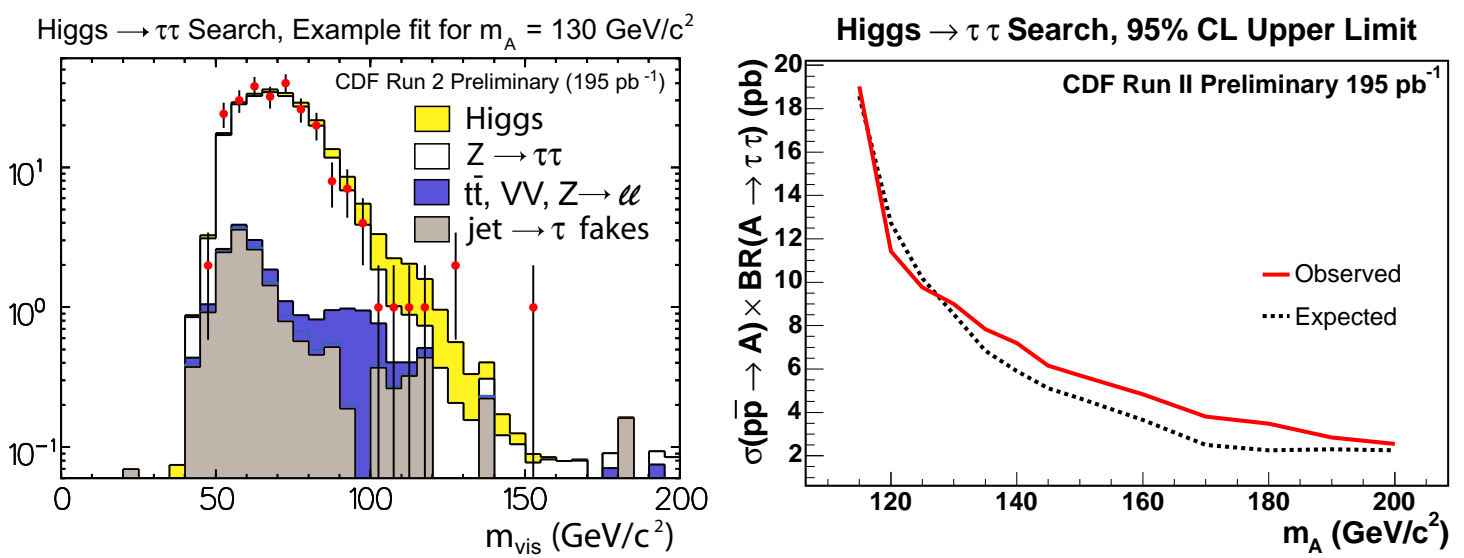

Figure 12: (Left) Distribution of the visible mass after all cuts of the CDF search for $\Phi \rightarrow \tau \tau$ in $195 \mathrm{pb}^{-1}$ of Run II data and for a Higgs boson signal with $m_{\Phi}=130 \mathrm{GeV} / \mathrm{c}^{2}$ on top of the Standard Model background; (Right) Upper limit (at 95\% C.L.) on the cross section $\sigma \cdot B R(\Phi \rightarrow \tau \tau)$ in comparison with the expected limit (from Ref. [63).

Both Tevatron experiments have demonstrated the ability to reconstruct hadronic tau decays in Run II data by measuring the $Z \rightarrow \tau \tau$ cross section. 62 The CDF collaboration has analyzed Run II data corresponding to an integrated luminosity of $195 \mathrm{pb}^{-1}$ in search for $g g \rightarrow \Phi \rightarrow \tau \tau$ with one tau decaying leptonically to electron or muon and the other tau decaying into hadrons $[63$ The hadronic tau decay is reconstructed as one or more tracks pointing to a narrow energy deposition in the calorimeter. Background from jets misreconstructed as tau objects is further suppressed using cuts on track multiplicity, mass and isolation of the tau candidate. The selection then requires one such tau candidate in addition to an isolated electron or muon. After topological cuts using the transverse momenta of the lepton and the hadronic tau candidate as well as the transverse missing energy, the sample is dominated by irreducible background from $Z \rightarrow \tau \tau$ with a fraction of about $90 \%$. Events from $\Phi \rightarrow \tau \tau$ are selected with an efficiency, including branching fractions, of about $0.8 \%(0.6 \%)$ in the electron (muon) channel for $m_{\Phi}=130 \mathrm{GeV} / \mathrm{c}^{2}$.

Separation of signal events from the $Z \rightarrow \tau \tau$ background is possible by reconstructing an event mass $m_{v i s}$ using the momentum vectors of the lepton and tau candidate as well as the missing transverse energy vector. In Figure 12(left) the distribution of $m_{\text {vis }}$ is shown for data, backgrounds and a potential Higgs signal. No evidence for an excess of events with respect to the Standard 

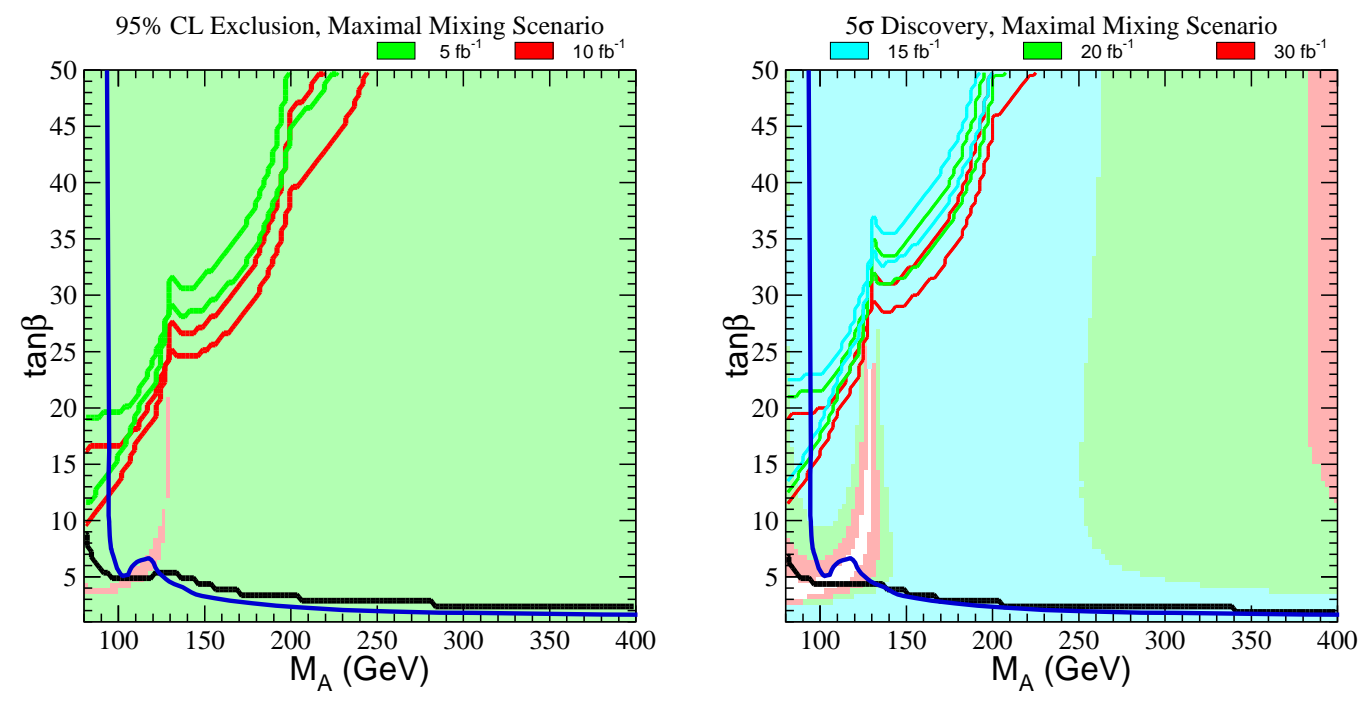

Figure 13: Luminosity required for exclusion at 95\% C.L. (left) or $5 \sigma$ discovery (right) of a SUSY Higgs boson as a function of $m_{A}$ and tan $\beta$ within the $m_{h}$-max scenario (taken from Ref. 36 and modified to include the most recent LEP2 limi $\left(\frac{13}{13}\right)$. Shaded regions indicate the reach of $W H$ and $Z H$ searches, the region above the diagonal lines are accessible to searches for $\Phi b(b)$, the dark line indicates the LEP2 limit.

Model prediction has been observed. Using a binned likelihood fit of this distribution, a limit on the production cross section of $\Phi \rightarrow \tau \tau$ has been extracted as displayed in Fig. 12 (right) as a function of the Higgs boson mass.

\subsubsection{Combined reach}

Combining dedicated searches for Higgs bosons at large $\tan \beta$ with searches for production of Higgs bosons in association with vector bosons, sensitivity at 95\% C.L. to MSSM Higgs bosons within the $m_{h}$-max scenario (see Section 7.4) can be achieved independent of $\tan \beta$, as shown in Fig. [13] [36] However, within this challenging scenario, a $5 \sigma$ discovery will not be possible at Tevatron Run II for most of the $\left(m_{A}, \tan \beta\right)$-plane.

\subsection{Charged Higgs bosons}

Models with an extended Higgs sector predict charged Higgs bosons $H^{ \pm}$or, in the case of additional Higgs triplets, also doubly-charged Higgs bosons $H^{ \pm \pm}$. For masses smaller than $m_{t}-m_{b}$, singlycharged Higgs bosons can be produced in top quark decays. In this case, charged Higgs bosons can be searched for either as an excess of events with $t \rightarrow H^{+} b$ or as a decrease in top-quark branching fraction for $t \rightarrow W^{+} b$. Limits on the mass of charged Higgs bosons as a function of $\tan \beta$ have been set at Run I using both strategies $\frac{16}{16}$ The reach of the Run I analyses has been projected to Run II employing a parametrized detector simulation: from measuring the branching fraction of leptonic top quark decays with $2 \mathrm{fb}^{-1}$ of data, charged Higgs boson masses of $80-140 \mathrm{GeV} / \mathrm{c}^{2}$ can be probed at $95 \%$ C.L. for $\tan \beta$ from $20-30$, respectively. Within supersymmetric models, this allows a test of the large $\tan \beta$ region complementary to the search for $\Phi b(b)$ and $\Phi \rightarrow \tau \tau$.

Preliminary Run II results of $t \bar{t}$ cross-section measurements by the CDF collaboration have been interpreted to obtain limits on the branching fraction of top quark decays into charged Higgs bosons. $\frac{64}{}$ This analysis is based on cross-section measurements in the dilepton, lepton plus jets and lepton plus hadronic-tau channel using Run II data corresponding to an integrated luminosity of $192 \mathrm{pb}^{-1}$. Assuming that charged Higgs bosons decay only into $c s, \tau \nu$ or $W b \bar{b}$, limits in the $\left(m_{H^{ \pm}}, \tan \beta\right)$-plane have been derived at tree level within the MSSM, as shown in Fig. 14.

Production of doubly charged Higgs bosons can provide particularly striking signatures in leftright symmetric models, $\frac{65]}{6 h e r e ~} \operatorname{BR}\left(H^{ \pm \pm} \rightarrow \ell^{ \pm} \ell^{ \pm}\right)$is expected to be $100 \%$. The $\mathrm{CDF}$ (DØ) 


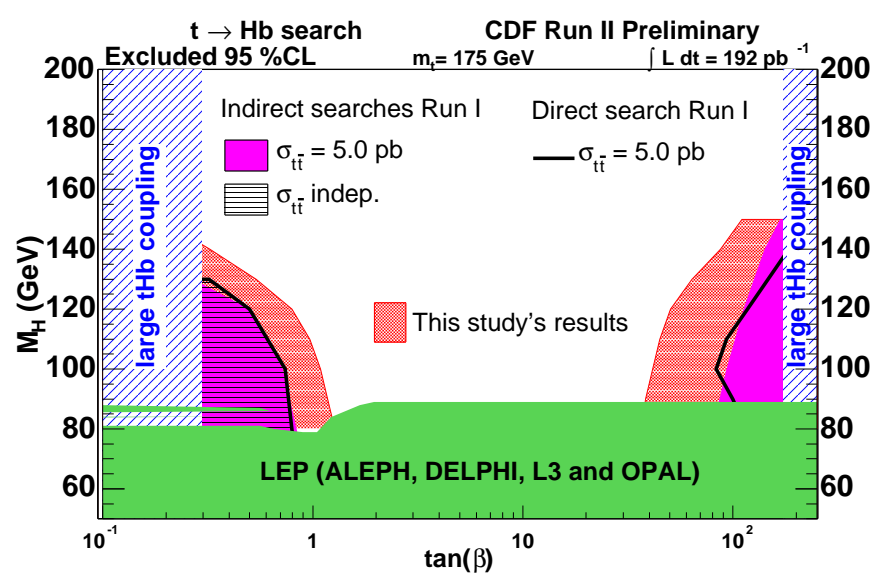

Figure 14: Regions in the MSSM ( $\left.m_{H^{ \pm}}, \tan \beta\right)$-plane excluded by the CDF Run II t $\bar{t}$ cross section measurements, in comparison with regions excluded at LEP and Run I; Tevatron results do not apply for non-perturbative Htb couplings at small and large $\tan \beta$ (from Ref. 64).

collaborations have analyzed Run II data corresponding to an integrated luminosity of $240 \mathrm{pb}^{-1}$ $\left(107 \mathrm{pb}^{-1}\right)$ to search for $H^{ \pm \pm}$production in like-sign dilepton events 66 Requiring two acoplanar, isolated electrons or muons, no excess of events has been observed at high dilepton masses. For left-handed (right-handed) $H^{ \pm \pm}$, the CDF collaboration sets a lower mass limit of $135 \mathrm{GeV} / \mathrm{c}^{2}$ $\left(112 \mathrm{GeV} / \mathrm{c}^{2}\right)$ for $\mathrm{BR}\left(H^{ \pm \pm} \rightarrow \ell^{ \pm} \ell^{ \pm}\right)=1$.

The searches described above loose sensitivity once the leptons are produced with significant impact parameter due to a non-negligible lifetime of the Higgs boson. Since the lifetime of doubly charged Higgs bosons depends on the unknown Yukawa coupling, scenarios with large $H^{ \pm \pm}$decay lengths have to be considered as well. The CDF collaboration has searched for long-lived doubly charged Higgs bosons that decay outside the detector in Run II data corresponding to an integrated luminosity of $206 \mathrm{pb}^{-1} 67$ Experimentally, these events can be identified requiring two isolated high$P_{T}$ tracks associated with hits in the muon detectors and large energy loss in the tracking detectors. Efficiencies of about 30\% have been achieved with negligible backgrounds from Standard Model sources. No events have been observed in the data, which translates into a lower limit on the $H^{ \pm \pm}$ mass of $134 \mathrm{GeV} / \mathrm{c}^{2}$.

\subsection{Summary of Higgs searches at the Tevatron}

With an integrated Run II luminosity of the order of $8 \mathrm{fb}^{-1}$, both Tevatron experiments combined are expected to reach sensitivity to the production of Standard Model Higgs bosons up to masses of $180 \mathrm{GeV} / \mathrm{c}^{2}$ at $95 \%$ C.L. Within the context of Supersymmetry, most of the parameter space can be tested at $95 \%$ C.L. by a combination of searches for Higgs bosons produced in association with vector bosons or $b$ quarks.

These estimates are based on analyses employing full detector simulation and reconstruction that has been tuned to Run II data. Preliminary results from searches using Run II data corresponding to an integrated luminosity of about $200 \mathrm{pb}^{-1}$ are available in the most important channels. The performance of the analyses is consistent with the projections, even though a number of assumptions about future improvements to reconstruction algorithms still remain to be verified. Due to the small amount of integrated luminosity that has been collected so far, the current results are not yet sensitive to Higgs boson production. Even with the full Run II luminosity and after combining all channels and both experiments, a $5 \sigma$ discovery will most likely not be possible at the Tevatron, leaving it to the LHC to explore and discover Higgs bosons over the full parameter range of both Standard Model and Supersymmetry. 


\section{The Search for a Standard Model Higgs Boson at the LHC}

The Standard Model Higgs boson is searched for at the LHC in various decay channels, the choice of which is given by the signal rates and the signal-to-background ratios in the different mass regions. The search strategies and background rejection methods have been established in many studies over the past ten years $\frac{6869}{69}$ Originally, inclusive final states have been considered, among them the well established $H \rightarrow \gamma \gamma$ and $H \rightarrow Z Z^{(*)} \rightarrow \ell \ell \ell \ell$ decay channels. More exclusive channels have been considered in the low mass region by searching for Higgs boson decays in $b \bar{b}$ or $\gamma \gamma$ in association with a lepton from a decay of an accompanying $W$ or $Z$ boson or a top quark. The search for a Standard Model Higgs boson in the intermediate mass region using the vector boson fusion mode and exploiting forward jet tagging, which had been proposed in the literature several years ago, 707172 has meanwhile been studied by the experimental collaborations. In the following, a brief summary of the Standard Model Higgs boson discovery potential at the LHC is given. After a discussion of the inclusive analyses, more exclusive final states are discussed, ordered according to the different production processes.

Despite the enormous progress in the calculation of higher order QCD corrections over the past few years (s. Section 2.1), the LHC physics performance generally has been evaluated by using Born-level predictions for both signals and backgrounds. Since the higher order QCD corrections (K-factors) are not known yet for all background processes this approach was considered to be more consistent and conservative. In the following K-factors are ignored, unless otherwise stated.

The non-diffractive inelastic proton-proton cross section has been assumed to be $70 \mathrm{mb}$. This leads on average to a superposition of 2.3 or 23 minimum bias events on top of the hard collision at low or high luminosity respectively. These so called pile-up contributions have been included for both low and high luminosity.

Physics processes have mainly been simulated with the PYTHIA Monte Carlo program,,$\frac{73}{1}$ including initial- and final-state radiation, hadronisation and decays. Although many results have been obtained using a fast simulation, all key performance characteristics have been evaluated with a full GEANT $\frac{48}{4}$ simulation, both at low and high luminosity $\underline{6869}$

\subsection{Inclusive Higgs boson searches}

Several important channels for Higgs boson discovery at the LHC have been discussed extensively in the literature. Among those channels are the $H \rightarrow \gamma \gamma$ decay mode, the gold plated decay channel $H \rightarrow Z Z^{(*)} \rightarrow 4 \ell$ as well as the decay channel $H \rightarrow W W^{(*)} \rightarrow \ell \nu \ell \nu$. If no additional particles except the Higgs boson decay products are searched for, the production via gluon fusion provides the largest contribution to the signal event yields.

\subsection{1 $H \rightarrow \gamma \gamma$ decays}

The decay $H \rightarrow \gamma \gamma$ is a rare decay mode, which is only detectable in a limited Higgs boson mass region between 80 and $150 \mathrm{GeV} / \mathrm{c}^{2}$, where both the production cross section and the decay branching ratio are relatively large. Excellent energy and angular resolution are required to observe the narrow mass peak above the irreducible prompt $\gamma \gamma$ continuum. In addition, there is a large reducible background resulting from direct photon production or from two-jet production via QCD processes. Using a realistic detector simulation, it has been demonstrated 6869 that the required rejection can be achieved and that the residual jet-jet and $\gamma$-jet backgrounds can be brought to the level of approximately $20 \%$ of the irreducible $\gamma \gamma$ background over the mass range relevant to the $H \rightarrow \gamma \gamma$ search. For an integrated luminosity of $100 \mathrm{fb}^{-1}$, a Standard Model Higgs boson in the mass range between 105 and $145 \mathrm{GeV} / \mathrm{c}^{2}$ can be observed in the $H \rightarrow \gamma \gamma$ channel in the ATLAS experiment with a significance of more than $5 \sigma \underline{68}$ The discovery range in the CMS experiment is slightly larger, due to the better $\gamma \gamma$ mass resolution. ${ }^{69}$ As an example of signal reconstruction above background, the expected signal from a Higgs boson with a mass of $130 \mathrm{GeV} / \mathrm{c}^{2}$ in the CMS experiment is shown in Fig. [15] assuming an integrated luminosity of $100 \mathrm{fb}^{-1}$.

The $\gamma \gamma$ decay mode has also been studied for the case where the Higgs boson is accompanied by a high- $P_{T}$ jet $\frac{7468}{6}$ a better signal-to-background ratio is found than in the inclusive case. However, 


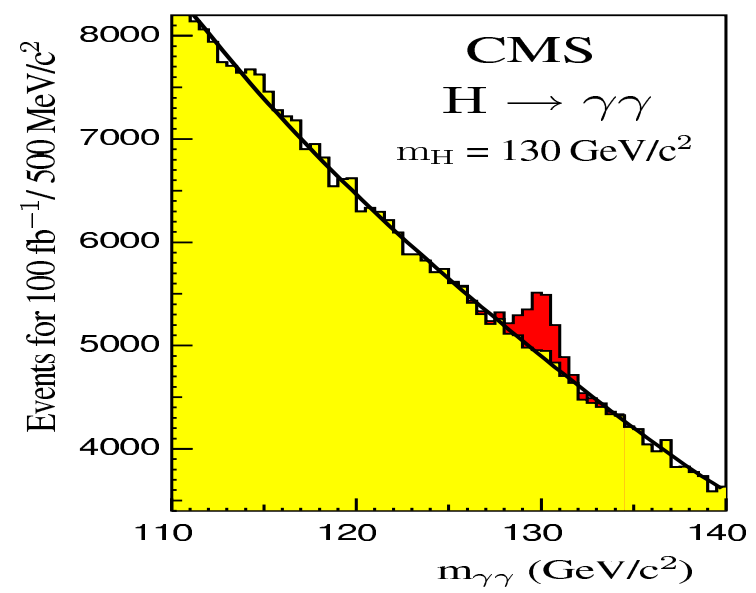

Figure 15: Reconstructed $\gamma \gamma$ invariant mass distribution of a $H \rightarrow \gamma \gamma$ signal (dark) with $m_{H}=130 \mathrm{GeV} / \mathrm{c}^{2}$ and the background (light) for an integrated luminosity of $100 \mathrm{fb}^{-1}$ in the CMS experiment (from Ref. 69).

sizeable uncertainties on the background estimates from Monte Carlo calculations prevent a precise estimate of the discovery significance at present.

\subsection{2 $H \rightarrow Z Z^{(*)}$ decays}

The decay channel $H \rightarrow Z Z^{*} \rightarrow \ell \ell \ell \ell$ provides a rather clean signature in the intermediate mass region $115 \mathrm{GeV} / \mathrm{c}^{2}<m_{H}<2 m_{Z}$. In addition to the irreducible backgrounds from $Z Z^{*}$ and $Z \gamma^{*}$ production, there are large reducible backgrounds from $t \bar{t}$ and $Z b \bar{b}$ production. Due to the large production cross section, the $t \bar{t}$ events dominate at production level, whereas the $Z b \bar{b}$ events contain a genuine $Z$ boson in the final state and are therefore more difficult to reject. In addition, there is background from $Z Z$ continuum production, where one of the $Z$ bosons decays into a $\tau$ pair, with subsequent leptonic decays of the $\tau$ leptons, and the other $Z$ decays into an electron or muon pair. It has been shown that in both LHC experiments the reducible backgrounds can be suppressed well below the level of the irreducible background from $Z Z^{*} \rightarrow 4 \ell$. Calorimeter and track isolation together with impact parameter measurements can be used to achieve the necessary background rejection. 6869 Assuming an integrated luminosity of $30 \mathrm{fb}^{-1}$, the $H \rightarrow Z Z^{*} \rightarrow 4 \ell$ signal can be observed with a significance of more than $5 \sigma$ in the mass range $130<m_{H}<180 \mathrm{GeV} / \mathrm{c}^{2}$, except for a narrow region around $170 \mathrm{GeV} / \mathrm{c}^{2}$, where the branching ratio is suppressed due to the opening up of the $W W$ decay mode (see Fig. 5 ).

It has also been studied whether $Z$-decay modes involving $b$ quarks can be used to enhance the signal significance. ${ }^{75}$ To take advantage of the fact that the branching ratio of $Z \rightarrow b \bar{b}$ is approximately five times larger than that of $Z \rightarrow \ell \ell$, a search for $H \rightarrow Z Z^{*} \rightarrow b \bar{b} \quad \ell^{+} \ell^{-}$has been considered, with the on-shell $Z$ boson decaying into a $b \bar{b}$ pair and the other decaying into a pair of electrons or muons. Since the large backgrounds from $t \bar{t}$ and $Z b \bar{b}$ production contain a genuine pair of $b$ quarks, the discovery potential of a Higgs boson in that channel is marginal. Assuming an integrated luminosity of $30 \mathrm{fb}^{-1}$, a signal significance of only $2.7 \sigma$ has been estimated with a signal-to-background ratio of about 0.3 for a Higgs boson with $m_{H}=150 \mathrm{GeV} / \mathrm{c}^{2}$.

For Higgs boson masses in the range $180 \mathrm{GeV} / \mathrm{c}^{2}<m_{H} \lesssim 700 \mathrm{GeV} / \mathrm{c}^{2}$, the $H \rightarrow Z Z \rightarrow 4 \ell$ decay mode is the most reliable one for the discovery of a Standard Model Higgs boson at the LHC. The expected background, which is dominated by the continuum production of $Z$ boson pairs, is smaller than the signal. In this mass range the Higgs boson width grows rapidly with increasing $m_{H}$, and dominates over the experimental mass resolution for $m_{H}>300 \mathrm{GeV} / \mathrm{c}^{2}$. The momenta of the final-state leptons are high and their measurement does not put severe requirements on the detector performance. The $H \rightarrow Z Z \rightarrow 4 \ell$ signal would be easily observable above the $Z Z \rightarrow 4 \ell$ continuum background after less than one year of low luminosity operation for $200<m_{H}<600 \mathrm{GeV} / \mathrm{c}^{2}$ ! $76 \mathrm{It}$ should be mentioned that the interference effect between the resonant signal and the non-resonant background, as discussed in Ref. 77, has not been taken into account. As an example of signal 
reconstruction above background, the expected signal from a Higgs boson with $m_{H}=300 \mathrm{GeV} / \mathrm{c}^{2}$ is shown in Fig. 16](left) for an integrated luminosity of only $10 \mathrm{fb}^{-1}$ in the ATLAS experiment.

For larger values of $m_{H}$, the Higgs boson signal becomes very broad and the signal rate drops rapidly. In the high mass region, the decay modes $H \rightarrow Z Z \rightarrow \ell \ell \nu \nu$ and $H \rightarrow Z Z \rightarrow \ell \ell j j$ provide additional discovery potentia $\left[\frac{6869}{6}\right.$ and allow to extend the $5 \sigma$-discovery range up to $\sim 1 \mathrm{TeV} / \mathrm{c}^{2}$.
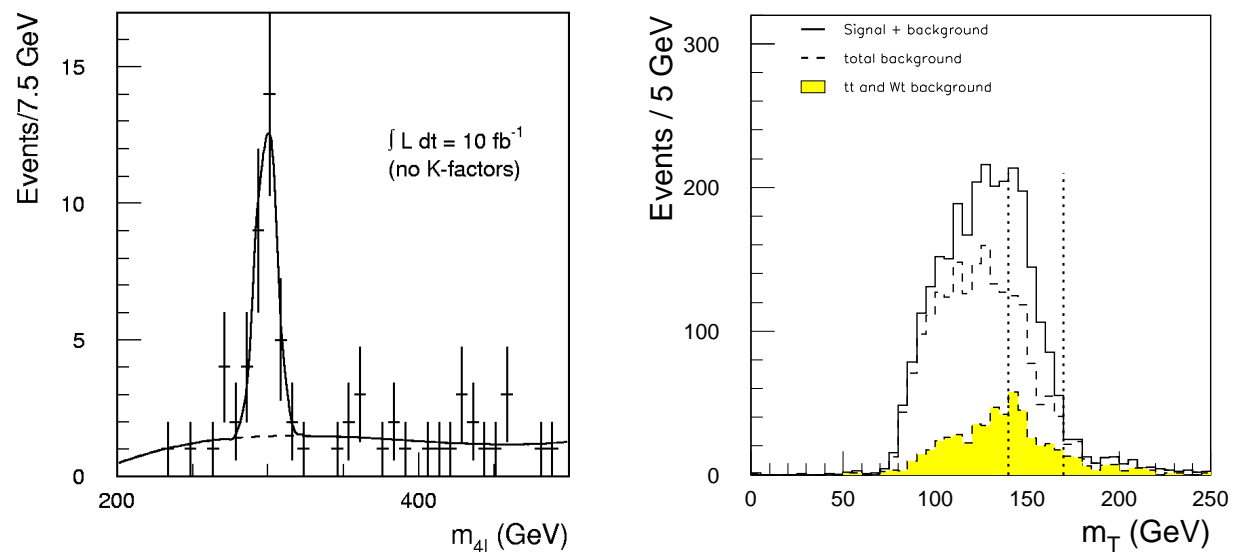

Figure 16: (Left) Expected $H \rightarrow Z Z \rightarrow 4 \ell$ signal above the background for $m_{H}=300 \mathrm{GeV} / \mathrm{c}^{2}$ and for an integrated luminosity of $10 \mathrm{fb}^{-1}$ in the ATLAS experiment (from Ref. 68). (Right) Transverse mass distribution for the summed $H \rightarrow W W^{*} \rightarrow \ell \nu \ell \nu$ signal $\left(m_{H}=150 \mathrm{GeV} / \mathrm{c}^{2}\right)$ and total background, for an integrated luminosity of $30 \mathrm{fb}^{-1}$. The distribution for the background alone is also shown separately. The shaded histogram represents the contributions from the $W t$ and $t \bar{t}$ backgrounds. The dashed lines indicate the selected signal region (from Ref. [68).

\subsection{3 $H \rightarrow W W^{(*)}$ decays}

For Higgs boson masses around $170 \mathrm{GeV} / \mathrm{c}^{2}$, for which the $Z Z^{*}$ branching ratio is suppressed, the discovery potential can be enhanced by searching for the $H \rightarrow W W^{(*)} \rightarrow \ell \nu \ell \nu$ decay! 5378 In this mode it is not possible to reconstruct a Higgs boson mass peak. Instead, an excess of events above the expected backgrounds can be observed and used to establish the presence of a Higgs boson signal. Usually, the transverse mass computed from the leptons and the missing transverse momentum, $m_{T}=\sqrt{2 P_{T}^{\ell \ell} E_{T}(1-\cos \Delta \varphi)}$, is used to discriminate between signal and background. The $W W$, $t \bar{t}$ and single-top production processes constitute severe backgrounds and the signal significance depends critically on their absolute knowledge. After relaxing cuts, the Monte Carlo predictions for those backgrounds can, however, be normalized to the data in regions where only a small fraction of signal events is expected. As an example, the distribution of the transverse mass is shown in Fig. 16(right) for the sum of signal plus background and for the background alone in the ATLAS experiment, assuming $m_{H}=170 \mathrm{GeV} / \mathrm{c}^{2}$ and an integrated luminosity of $30 \mathrm{fb}^{-1}$. Under the assumption that the total background is known with an uncertainty of $\pm 5 \%$, a Higgs boson signal can be extracted with a significance of more than $5 \sigma$ for $\sim 150<m_{H}<190 \mathrm{GeV} / \mathrm{c}^{2}$ for an integrated luminosity of $30 \mathrm{fb}^{-1}$.

For Higgs boson masses beyond $\sim 800 \mathrm{GeV} / \mathrm{c}^{2}$ the decay mode $H \rightarrow W W \rightarrow \ell \nu j j$ provides additional discovery potential. 6869 The branching ratio is about 150 times larger than for the $H \rightarrow Z Z \rightarrow 4 \ell$ decay channel, thus providing the largest possible signal rate with at least one charged lepton in the final state. An important contribution to the production cross section of such a heavy Higgs boson is the vector boson fusion process, resulting in the production of two final state quark jets in the high rapidity regions in association with the Higgs boson (see Section [5.2). This production mechanism provides an important additional signature for isolating the signal from the background.

The experimental challenge in the reconstruction of these events is twofold: (i) the reconstruction of high- $P_{T} \mathrm{~W}$ decays to two jets, and (ii) the tagging of the forward jets in the presence of large pile-up. As an example, for a Higgs boson mass of $1 \mathrm{TeV} / \mathrm{c}^{2}$ and an integrated luminosity of 
$100 \mathrm{fb}^{-1}$, about 60 signal events above a background of 20 events are expected. However, there is no substantial difference between the shapes of the signal and background distributions and some years of running may be needed before a signal could be established.

\subsection{Higgs boson searches using vector boson fusion}

In recent studies it has been demonstrated that, not only in the high mass but also in the intermediate mass range, the discovery potential can be significantly increased by performing a search for Higgs boson production in the vector boson fusion mode.7071/721798069 Although the contribution to the cross section in the intermediate mass range amounts at leading order only to about $20 \%$ of the total production cross section, the additional event characteristics can be exploited to suppress the large backgrounds. In vector boson fusion events, the Higgs boson is accompanied by two jets in the forward regions of the detector, originating from the initial quarks that emit the vector bosons. On the other hand, central jet activity is suppressed due to the lack of colour exchange between the initial state quarks. This is in contrast to most background processes, where colour flow appears in the $t$-channel. Jet tagging in the forward region of the detector together with a veto of jet activity in the central region are therefore powerful tools to enhance the signal-to-background ratio.

The performance of the detectors for forward jet tagging has been studied in a detailed simulation.7969 In the study presented in Ref. [79, the two tag jets are searched for over the full calorimeter coverage $(|\eta|<4.9)$. The jets with the highest $P_{T}$ in the positive and negative regions of pseudorapidity are taken to be the tag jet candidates. Each jet is required to have a transverse energy of at least $20 \mathrm{GeV}$. The pseudorapidity distribution and the separation $\Delta \eta$ between the two tag jets, as found from the parton-level information, is shown in Fig. 17f for signal events with $m_{H}=160 \mathrm{GeV} / \mathrm{c}^{2}$. For the tagging algorithm described above, distributions at parton and reconstruction level are in good agreement. For comparison, the corresponding distributions for tag jets as reconstructed in $t \bar{t}$ background events are superimposed on the figure. From these distributions it can be concluded that a large pseudorapidity separation can be used for the discrimination between signal and backgrounds from QCD production.
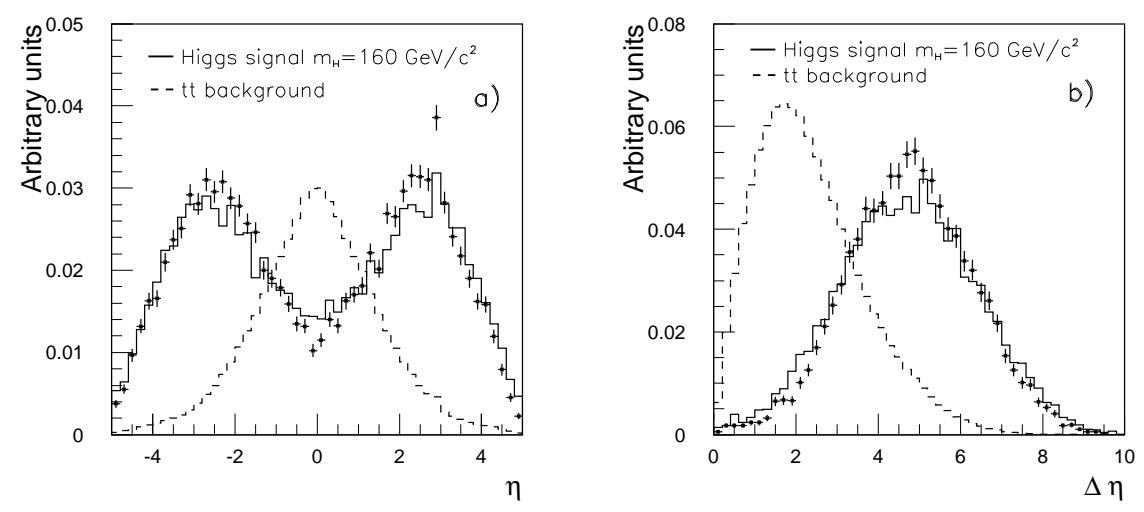

Figure 17: (Left) Pseudorapidity distribution of the tag jets in signal events with $m_{H}=160 \mathrm{GeV} / \mathrm{c}^{2}$ and for $t \bar{t}$ background events. (Right) Separation $\Delta \eta$ between the tag jets for the same types of events. The full histograms show the distributions at parton level, the dots represent the distributions at reconstruction level, after the tagging algorithm has been applied. The corresponding distributions for jets identified as tag jets in $t \bar{t}$ events are superimposed as dashed histograms. All distributions are normalized to unity (from Ref. [79).

The detailed simulation performed in Ref. 81 has demonstrated that tag jets can be reliably reconstructed in the ATLAS detector. The efficiency for reconstructing a tag jet in signal events with $P_{T}$ above $20 \mathrm{GeV} / \mathrm{c}$ is shown in Fig. 18(left) as a function of pseudorapidity $\eta$.

Generally, the fast simulation package of the ATLAS detector 82 provides a sufficiently good description of the tagging efficiency. Differences between the fast and full simulation have been found in the transition regions between different calorimeters and at very forward rapidities. These differences have been parametrized as a function of $P_{T}$ and $\eta$ to incorporate them in the fast simulation. 81 
As pointed out above, a veto against jets in the central region will be an important tool to suppress background from QCD processes. It should be noted that a reliable estimate of the jet veto efficiency is difficult to obtain. Present estimates are based on leading-order parton shower Monte Carlos and might be affected by sizeable uncertainties. It is important to explore new Monte Carlo approaches 108 and to compare their predictions to Tevatron data.

At the LHC, jets in the central region can be produced also by pile-up events. In the full simulation study it has been found that after applying a threshold cut on the calorimeter cell energies of $0.2 \mathrm{GeV}$ at low and $1.0 \mathrm{GeV}$ at high luminosity, the veto rate due to fake jets from pile-up events can be kept at a low level, provided that $P_{T}$ thresholds of $20 \mathrm{GeV} / \mathrm{c}$ at low and $30 \mathrm{GeV} / \mathrm{c}$ at high luminosity are used for the jet definition. The results of this study are presented in Fig. 18(right), where the efficiency to find a jet from pile-up events in different intervals of central rapidity is shown as a function of the jet $P_{T}$-threshold for low and high luminosity.
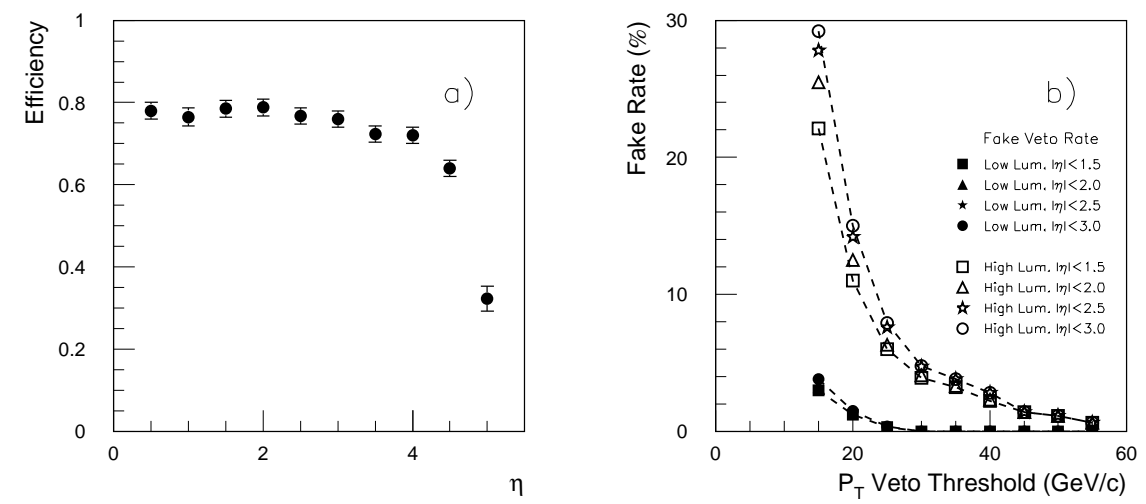

Figure 18: (Left) Efficiency for reconstructing a tag jet with $P_{T}>20 \mathrm{GeV} / \mathrm{c}$ which originates from a parton with $P_{T}>20 \mathrm{GeV} / \mathrm{c}$ as a function of pseudorapidity $\eta$ of the parton. (Right) Probability for finding at least one jet from pile-up events in central rapidity intervals in the ATLAS detector as a function of jet $P_{T}$ threshold. The dashed curves connect the points for pseudorapidity itervals $|\eta|<1.5$ and $|\eta|<3.0$ for low and high LHC luminosities.

The Higgs boson search in the vector boson fusion mode for various final states is discussed in the following.

\subsection{1 $q q H \rightarrow q q W W^{(*)}$}

According to Monte Carlo studies, the LHC experiments have a large discovery potential in the $H \rightarrow W W^{(*)} \rightarrow \ell^{+} \ell^{-} \not P_{T}$ decay mode ${ }^{83[84}$ The additional signatures of tag jets in the forward and of a low jet activity in the central regions of the detector allow for a significant reduction of the background. This results in a better signal-to-background ratio compared to the inclusive $H \rightarrow W W^{(*)}$ channel, which is dominated by the gluon fusion process. As a consequence, the signal sensitivity is less affected by systematic uncertainties on the predictions of the background. As an example, the reconstructed transverse mass distribution for a Higgs boson signal with a mass of $160 \mathrm{GeV} / \mathrm{c}^{2}$ is shown on top of the backgrounds from $t \bar{t}, W t$, and $W W$ production in Fig. 19.(left). This figure demonstrates the better signal-to-background ratio as compared to the inclusive case shown in Fig. 16(right). Since neutrinos appear in the final state, the transverse mass, defined as

$$
M_{T}=\sqrt{\left(E_{T}^{\ell \ell}+E_{T}^{\nu \nu}\right)^{2}-\left(\vec{p}_{T}^{\ell \ell}+\vec{P}_{T}\right)^{2}}
$$

is used for the mass reconstruction. For Higgs boson masses below $\sim 2 m_{W}$, the $W$ bosons are mostly at rest in the Higgs boson centre-of-mass system, resulting in $m_{\ell \ell}=m_{\nu \nu}$, such that for both the dilepton and the neutrino system the transverse energy can be calculated as 70

$$
E_{T}^{\ell \ell}=\sqrt{\left(P_{T}^{\ell \ell}\right)^{2}+m_{\ell \ell}^{2}} \quad \text { and } \quad E_{T}^{\nu \nu}=\sqrt{\left(P_{T}\right)^{2}+m_{\ell \ell}^{2}} .
$$



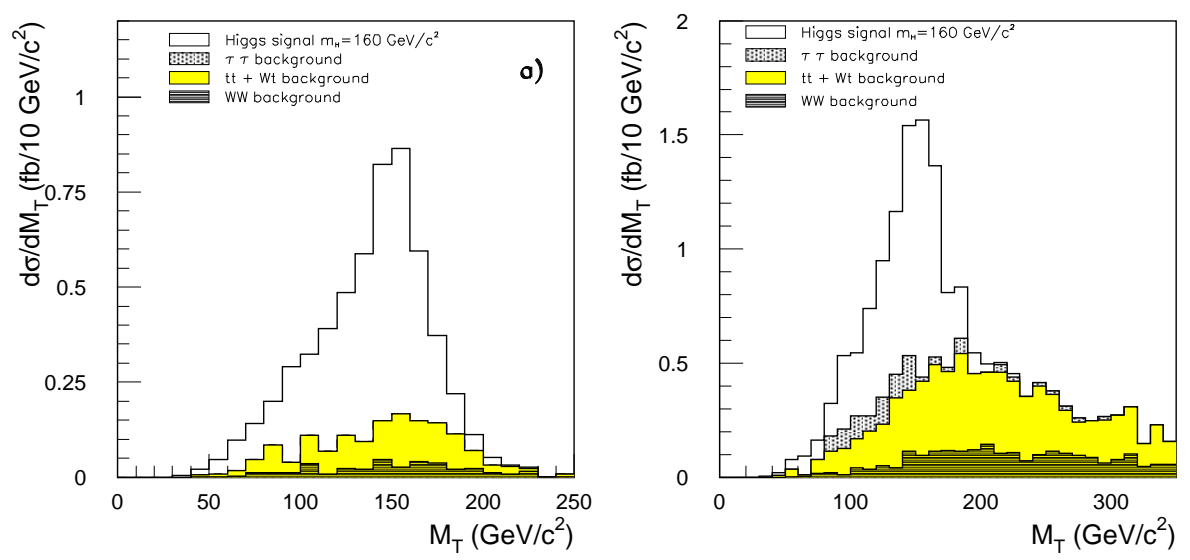

Figure 19: (Left) Distribution of the transverse mass $M_{T}$ for a Higgs boson with a mass of

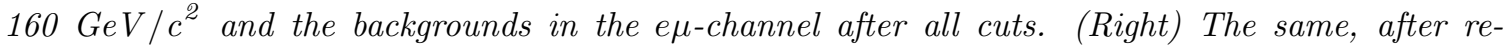
laxing kinematical cuts on the reconstructed leptons. The accepted cross sections $d \sigma / d M_{T}$ (in fb/10 GeV $/ \mathrm{c}^{2}$ ) including all efficiency and acceptance factors are shown in both cases (from Ref. [79).

After all cuts, a signal cross section in the $e \mu$ channel for a Higgs boson with a mass of $160 \mathrm{GeV} / \mathrm{c}^{2}$ of the order of $4.6 \mathrm{fb}$ is expected above a total background expectation of $1.2 \mathrm{fb}$. Due to this large signal-to-background ratio, this channel alone has a good discovery potential for a Higgs boson with a mass around $160 \mathrm{GeV} / \mathrm{c}^{2}$ and is not very sensitive to systematic uncertainties on the background. This compares favourably with the $g g \rightarrow W W^{(*)}$ channel discussed in Section 5.1.3 It is interesting to note that an application of looser kinematical cuts on the final-state leptons allows for a better discrimination between the signal and background shape 79 The corresponding transverse mass distribution is shown in Fig. 19(right). In this case, the background extends to higher $M_{T}$ values and a background normalization outside the signal region is possible.

The presence of a signal can also be demonstrated in the distribution of $\Delta \phi$, the difference in azimuthal angle between the two leptons in the final state. This distribution is shown in Fig. 20 for $e \mu$ final states passing the looser cuts, i.e., without cuts on the spatial separation of the leptons. Depending on $M_{T}$ the event sample has been split into two subsamples: (a) in the so called signal sample $\left(M_{T}<175 \mathrm{GeV} / \mathrm{c}^{2}\right)$ and (b) in a control sample $\left(M_{T}>175 \mathrm{GeV} / \mathrm{c}^{2}\right)$. For events in the signal region (Fig. 20(left)), a pronounced structure at small $\Delta \phi$ is seen, as expected for a spin-0 resonance. This behaviour is not present for events in the control sample (Fig. 20(right)), where the $t \bar{t}$ and $W W$ backgrounds are expected to dominate. Therefore, the unbiased $\Delta \phi$ distribution, resulting from relaxed kinematical cuts, can be used for both a demonstration of the consistency of the signal with a spin-0 hypothesis and for an additional background normalization. This normalization can be performed in the high $\Delta \phi$ region using events directly below the peak.

In addition, it has been studied whether the larger hadronic branching ratio of the $\mathrm{W}$ boson can be exploited and the process $q q \rightarrow q q H \rightarrow q q W W^{(*)} \rightarrow q q \ell \nu q q$ can be detected. 86 The cross section times branching ratio is about 4.3 times larger than for the dilepton channel. However, since only one lepton is present in the final state, $W+$ jet production is a serious additional background, with a cross section more than two orders of magnitude larger than the signal cross section. A detailed study has shown that after all cuts the signal rates and the signal-to-background ratio are much lower than the corresponding numbers in the dilepton channel. For a Higgs boson mass of $160 \mathrm{GeV} / \mathrm{c}^{2}$ and an integrated luminosity of $30 \mathrm{fb}^{-1}$, for example, 24 signal and 18 background events are expected. 86 The estimate of the dominant $W+$ jet background has been performed using a tree-level Monte Carlo generator. ${ }^{87}$ Given these results, this channel should not be considered a Higgs boson discovery channel, but could be used to confirm an observation of a Higgs boson with a mass around $160 \mathrm{GeV} / \mathrm{c}^{2}$.

In addition, it has been shown in Ref. 85 that the $q q \rightarrow q q H \rightarrow q q W W^{(*)} \rightarrow q q \ell \nu \ell \nu$ decay mode also increases the Higgs boson discovery potential in the high mass region between $\sim 300 \mathrm{GeV} / \mathrm{c}^{2}$ and $\sim 600 \mathrm{GeV} / \mathrm{c}^{2}$. 

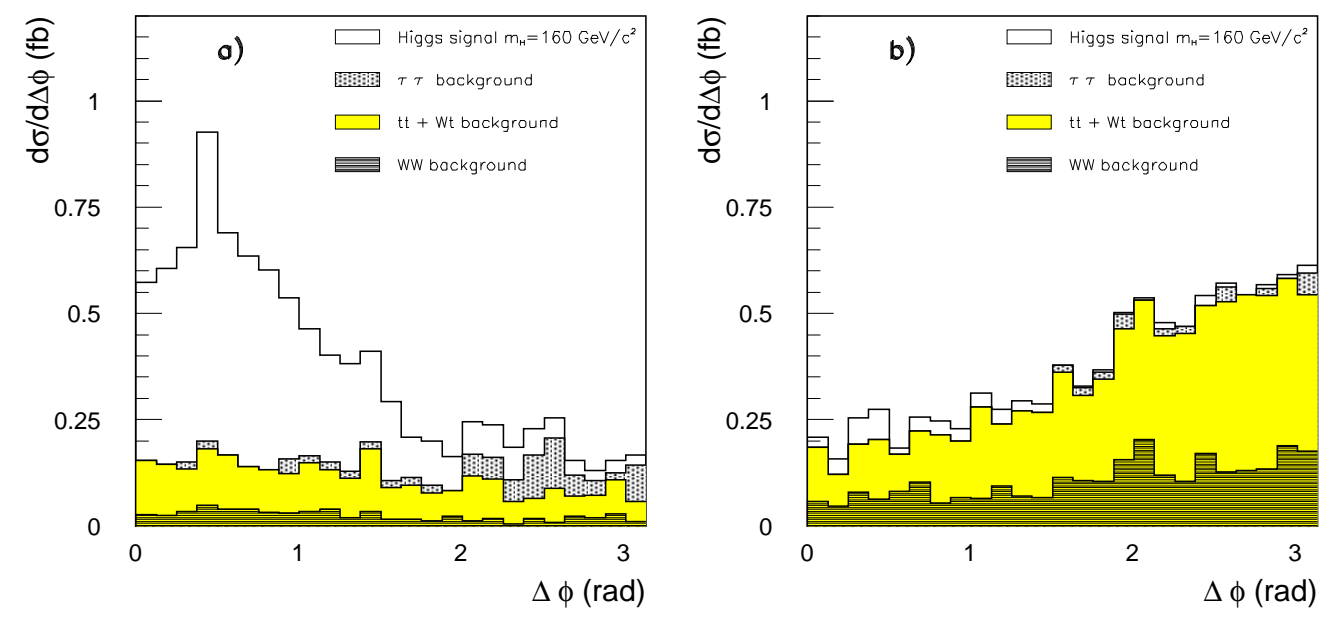

Figure 20: Distributions of the azimuthal opening angle $\Delta \phi$ between the two leptons for events in the signal region $\left(M_{T}<175 \mathrm{GeV} / \mathrm{c}^{2}\right)$ (left) and events outside the signal region $\left(M_{T}>175 \mathrm{GeV} / \mathrm{c}^{2}\right.$ ) (right). The accepted cross sections $d \sigma / d \Delta \phi$ (in fb/0.13 rad) including all efficiency and acceptance factors are shown in both cases (from Ref. 79).

\subsection{2 $\quad q q H \rightarrow q q \tau \tau$}

It has been shown that in the mass region $110<m_{H}<140 \mathrm{GeV} / \mathrm{c}^{2}$ the ATLAS and CMS experiments are sensitive to the $\tau \tau$ decay mode of the Standard Model Higgs boson in the vector boson fusion channel $\frac{8088}{80}$ Searches for $H \rightarrow \tau \tau$ decays using the double leptonic decay mode $q q H \rightarrow q q \tau \tau \rightarrow q q \ell \nu \bar{\nu} \ell \bar{\nu} \nu$ and the lepton-hadron decay mode $q q H \rightarrow q q \tau \tau \rightarrow q q \ell \nu \nu$ had $\nu$ seem to be feasible. In these analyses, $Z$ +jet production with $Z \rightarrow \tau \tau$ constitutes the principal background. The $\tau \tau$ invariant mass can be reconstructed using the collinear approximation. In signal and background events, the $H$ and $Z$ bosons are emitted with significant $P_{T}$, which contributes to large tau boosts and causes the tau decay products to be nearly collinear in the laboratory frame. Assuming that the tau directions are given by the directions of the visible tau decay products (leptons or hadrons from tau decays respectively), the tau momenta and therefore the $\tau \tau$ invariant mass can be reconstructed. As an example, distributions of the reconstructed $\tau \tau$ invariant mass for the $e \mu$ and for the $\ell$-had final states are shown in Fig. 21 for Higgs boson masses of $120 \mathrm{GeV} / \mathrm{c}^{2}$ and $135 \mathrm{GeV} / \mathrm{c}^{2}$. A discovery based on a combination of these final states would require an integrated luminosity of about $30 \mathrm{fb}^{-1}$. It should however be stressed that this assumes that the background from $Z \rightarrow \tau \tau$ decays in the signal region is known with a precision of $\pm 10 \%$. More studies are needed to establish that this precision can indeed be achieved. The detection of the $H \rightarrow \tau \tau$ decay mode is particularly important for a measurement of the Higgs boson couplings to fermions and for Higgs boson searches in MSSM scenarios, as discussed in Sections 6.3 and 7.4

\subsection{3 $\quad q q H \rightarrow q q \gamma \gamma$}

In addition, the prospects for discovering a Standard Model Higgs boson in the $H \rightarrow \gamma \gamma$ decay mode have been evaluated. 8990 Due to the small $H \rightarrow \gamma \gamma$ decay branching ratio the expected signal rate in the vector boson fusion mode is small. As in the inclusive analysis, there are significant backgrounds from non-resonant $\gamma \gamma$ production with jets and from QCD multijet and direct photon production. In the analysis,, 90 the $\gamma \gamma j j$ (QCD and electroweak), $\gamma j j j$ and $j j j j$ matrix elements have been considered. After applying a similar selection as in the inclusive case and requiring tagging jets with a large separation in pseudorapidity, a signal significance of $2.2 \sigma$ for $m_{H}=130 \mathrm{GeV} / \mathrm{c}^{2}$ has been found assuming an integrated luminosity of $30 \mathrm{fb}^{-1}$. Although the significance is lower than in the inclusive case, a much larger signal-to-background ratio with values around 0.5 can be reached. On the other hand, this decay mode appears to be considerably more sensitive to the background resulting from QCD processes with jets misidentified as photons. 

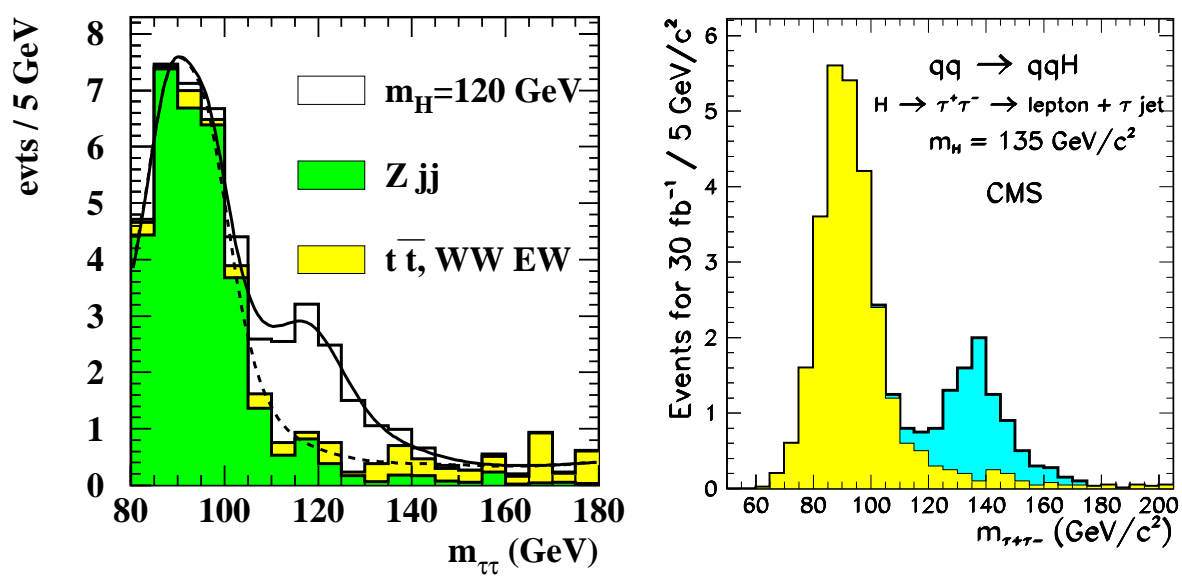

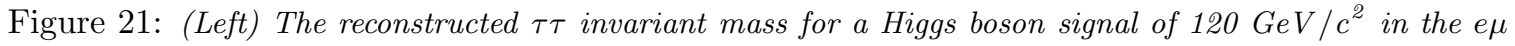
channel above all backgrounds after application of all cuts except the mass window cut around the Higgs boson mass. The number of signal and background events are shown for an integrated luminosity of $30 \mathrm{fb}^{-1}$ (from Ref. 79). (Right) The same, for the $\ell$-had channel and for a Higgs boson with $m_{H}=135 \mathrm{GeV} / \mathrm{c}^{2}$ (from Ref. 69).

\subsection{4 $q q H \rightarrow q q Z Z^{(*)}$}

The $H \rightarrow Z Z \rightarrow \ell \ell q q$ mode has been studied for Higgs boson masses larger than $2 m_{Z} \cdot 91$ In that channel, the background is largely dominated by the $Z+4$ jet production with $Z \rightarrow \ell \ell$. For a Higgs boson with $m_{H}=300 \mathrm{GeV} / \mathrm{c}^{2}$, a signal significance of $3.8 \sigma$ has been estimated, assuming an integrated luminosity of $30 \mathrm{fb}^{-1}$. Also in this case, the signal significance appears to be weaker than for the standard $H \rightarrow 4 \ell$ channel and the estimated background cross sections are subject to large theoretical uncertainties.

\subsection{5 $\quad q q H \rightarrow q q b \bar{b}$}

The detection of the fully hadronic decay mode $q q H \rightarrow q q b \bar{b}$ is extremely challenging in a hadron collider environment, given the huge background from QCD jet production. Already at the first trigger level the acceptance for signal events is low, since it is foreseen to run the experiments with relatively high $E_{T}$ thresholds for pure jet triggers. ${ }^{92}$ Even if the trigger issue is ignored, a very low signal-to-background ratio is expected. First parton level studies 9394 have shown that the dominant $b b j j$ background is about 300 times larger than the signal. To claim a signal, the background must be known with accuracies at the per mille level, which will be very hard to achieve. Studies using a realistic trigger simulation are still in progress.

\subsubsection{Discovery potential in the vector boson fusion channels}

The vector boson fusion channels provide a large discovery potential even for small integrated luminosities. The expected signal significance in the mass region $110<m_{H}<190 \mathrm{GeV} / \mathrm{c}^{2}$ is shown in Fig. 22 for the ATLAS experiment for the two main channels for an integrated luminosity of only $10 \mathrm{fb}^{-1}$. Combining the two channels, a Standard Model Higgs boson can be discovered with a significance above $5 \sigma$ in the mass range 130 to $190 \mathrm{GeV} / \mathrm{c}^{2}$, assuming a systematic uncertainty of $10 \%$ on the background. If the vector boson fusion channels are combined with the Higgs boson discovery channels discussed in Sections [5.1 and 5.3 the $5 \sigma$ discovery range, for that value of the integrated luminosity, can be extended down to $\sim 120 \mathrm{GeV} / \mathrm{c}^{2}$. According to these expectations, a Higgs boson discovery at the LHC should be possible in the low mass region in each experiment after about one year of running at low luminosity, provided the detectors are well understood in terms of lepton identification, $\mathbb{E}_{T}$-resolution and forward-jet tagging. 


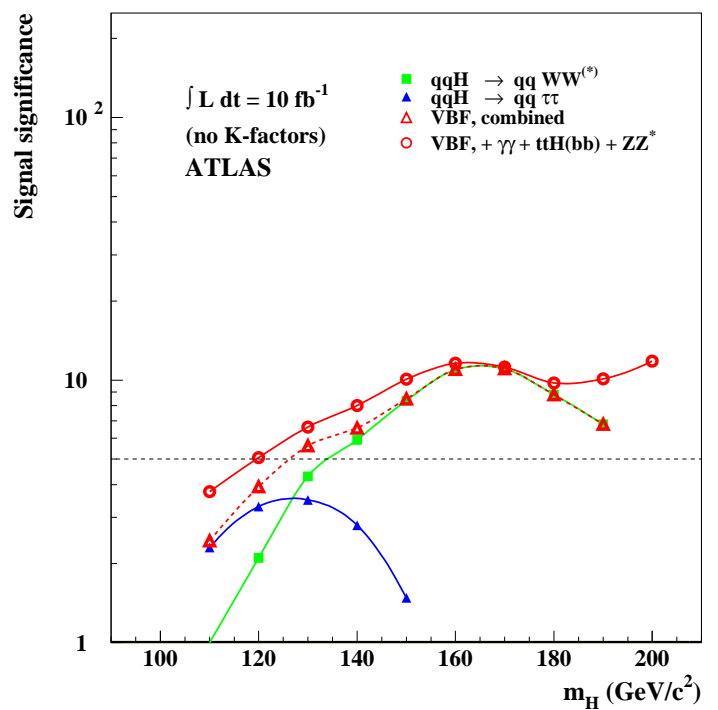

Figure 22: ATLAS sensitivity for the discovery of a Standard Model Higgs boson for an integrated luminosity of $10 \mathrm{fb}^{-1}$ in the low mass region. The signal significances are plotted for individual channels, as well as for the combination of several channels. A systematic uncertainty of $\pm 10 \%$ on the background has been included for the vector boson fusion channels (from Ref. 79).

\subsection{Higgs boson searches using the associated $W H, Z H$ and $t \bar{t} H$ produc- tion}

\subsection{1 $\quad H \rightarrow \gamma \gamma$ decays}

The $\gamma \gamma$ decay mode has also been studied for the associated production of a Higgs boson with a $W$ or $Z$ boson or a $t \bar{t}$ pair $[6869$ In both production modes at least one additional lepton is required from the vector boson or top-quark decay. Those channels have been found to have a better signalto-background ratio than the inclusive $H \rightarrow \gamma \gamma$ channel, e.g., assuming an integrated luminosity of $100 \mathrm{fb}^{-1}, 13.2$ signal events for $m_{H}=120 \mathrm{GeV} / \mathrm{c}^{2}$ are expected in the ATLAS experiment from the sum of $W H, Z H$ and $t \bar{t} H$ production above a total background of 5.7 events. 68 The background is dominated by irreducible contributions from $W \gamma \gamma, Z \gamma \gamma$ and $t \bar{t} \gamma \gamma$ production, which sum up to an irreducible fraction of $70-80 \%$. The statistical significance is found to be around $4.3 \sigma$ for masses in the range between 100 and $120 \mathrm{GeV} / \mathrm{c}^{2}$. An observation in this channel would therefore represent an independent confirmation of a possible discovery of a light Higgs boson at the LHC.

If the accompanying $W$ boson or one of the top quarks decays leptonically, a neutrino leading to missing transverse energy is present in the final state. In addition, missing transverse energy appears in the decay mode $Z H \rightarrow \nu \nu \gamma \gamma$. In a recent study it has been investigated whether an accompanying missing transverse energy signature (instead of an additional lepton) can be used to establish a $H \rightarrow \gamma \gamma$ signal!95

In the analysis, the same photon selection as in the inclusive case is applied. In addition, large missing transverse energy, $E_{T}>66 \mathrm{GeV} / \mathrm{c}^{2}$ is required. For an integrated luminosity of $100 \mathrm{fb}^{-1}$ and a Higgs boson with a mass of $120 \mathrm{GeV} / \mathrm{c}^{2}$, a signal of 20.9 events above a background of only 5.4 events is expected. The distribution of the invariant $\gamma \gamma$ mass is shown in Fig. 23)(left). The signal is dominated by contributions from the $W H+Z H(45 \%)$ and $t \bar{t} H(50 \%)$ associated production. Due to the $E_{T}$ requirement the $g g \rightarrow H \rightarrow \gamma \gamma$ process accounts for only $5 \%$ of the selected signal events, despite the much larger production cross section. Likewise a large fraction of the irreducible $\gamma \gamma$ background is rejected. The residual background is dominated by events from $Z \gamma \gamma$ and $W \gamma \gamma$ production. The background has been estimated using a tree-level Monte Carlo generator 96 and a fast simulation of the ATLAS detector performance. ${ }^{82}$ It should be stressed that in this simulation non-Gaussian tails in the $\mathbb{E}_{T}$ distribution, which might arise from instrumental effects, are not taken 
into account.

Additional cuts allow to disentangle the various contributions to the signal, which is important for the determination of the Higgs boson couplings to fermions and bosons (see Section 6.3). An additional lepton veto, for example, rejects $W H$ and $t \bar{t} H$ events and selects preferentially $Z H \rightarrow$ $\nu \nu \gamma \gamma$ events. An additional jet veto leads to a strong suppression of $t \bar{t} H$ events $\frac{95}{}$
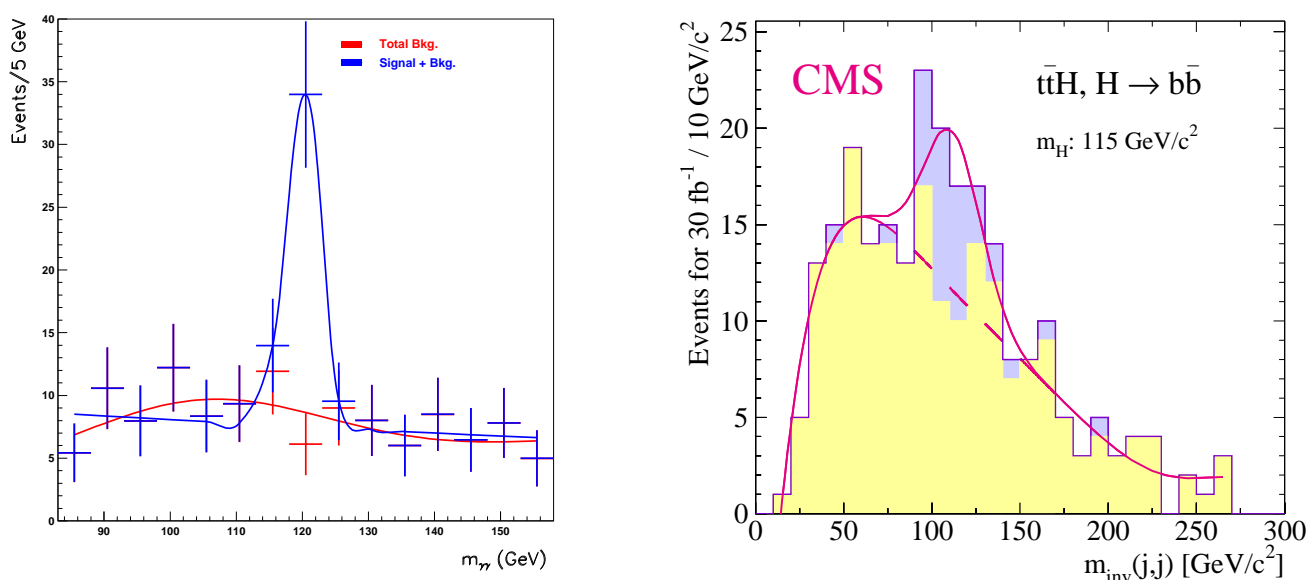

Figure 23: (Left) Reconstructed $\gamma \gamma$ invariant mass distribution after the $\gamma \gamma+E_{T}$ selection for an integrated luminosity of $100 \mathrm{fb}^{-1}$ (from Ref. 95). (Right) Reconstructed b $\bar{b}$ invariant mass distribution of a ttH $\rightarrow$ $\ell \nu b q q b$ bb signal (dark) with $m_{H}=115 \mathrm{GeV} / \mathrm{c}^{2}$ and the background (light) for an integrated luminosity of $30 \mathrm{fb}^{-1}$ (from Ref. 100).

\subsection{2 $\quad H \rightarrow b \bar{b}$ decays}

At low mass, the associated $H \rightarrow \gamma \gamma$ channel is nicely complemented by a search for the decay mode $H \rightarrow b \bar{b}$, which has the largest branching ratio in this mass range. Due to the huge backgrounds from QCD jet production in this decay mode, only the associated production modes have sensitivity. It has been demonstrated that the discovery potential for a Standard Model Higgs boson in the $W H$ production mode at the LHC is marginal 689798 It is limited by large backgrounds from $W b \bar{b}$, $W q \bar{q}$, and $t \bar{t}$ production. For small integrated luminosities, the extraction of a signal appears to be very difficult, even under the most optimistic assumptions for b-tagging performance and calibration of the shape and magnitude of the various backgrounds from data itself. If backgrounds are well known, evidence for a signal in this channel may be reached at large integrated luminosities, $\frac{99}{9}$ and valuable information can be provided for the measurement of the Higgs boson coupling to vector bosons.

On the contrary, the extraction of a Higgs boson signal in the $t \bar{t} H, H \rightarrow b \bar{b}$ channel appears to be feasible in the low Higgs boson mass region.10010198 Here it is assumed that the two top-quark decays can be fully reconstructed with an acceptable efficiency, which calls for an excellent b-tagging capability of the detector. Another critical item is the knowledge of the shape of the main residual background from $t \bar{t} j j$ production. If the shape can be accurately determined using real data from $t \bar{t}$ production, a Higgs boson signal could be extracted with a significance of more than $5 \sigma$ in the mass range from 80 to $120 \mathrm{GeV} / \mathrm{c}^{2}$, assuming an integrated luminosity of $30 \mathrm{fb}^{-1}$. As an example, the reconstructed invariant $b \bar{b}$ mass distribution for the $t \bar{t} H \rightarrow \ell \nu b q q b b b$ signal with $m_{H}=115 \mathrm{GeV} / \mathrm{c}^{2}$ and background events is shown in Fig. 23(right) for the CMS experiment, assuming an integrated luminosity of $30 \mathrm{fb}^{-1}$.

In addition, the channels $Z H \rightarrow \ell \ell b \bar{b}, Z H \rightarrow \nu \nu b \bar{b}$ and $b \bar{b} H \rightarrow b \bar{b} b \bar{b}$ have been suggested in the literature for Higgs boson searches in the $b \bar{b}$ decay mode ${ }^{102}$ For $Z H \rightarrow \ell \ell b \bar{b}$ a similar signalto-background ratio is expected as for the $W H$ channel $[68$ The other two have so far not been considered by the LHC collaborations, due to the challenging trigger and background conditions. 


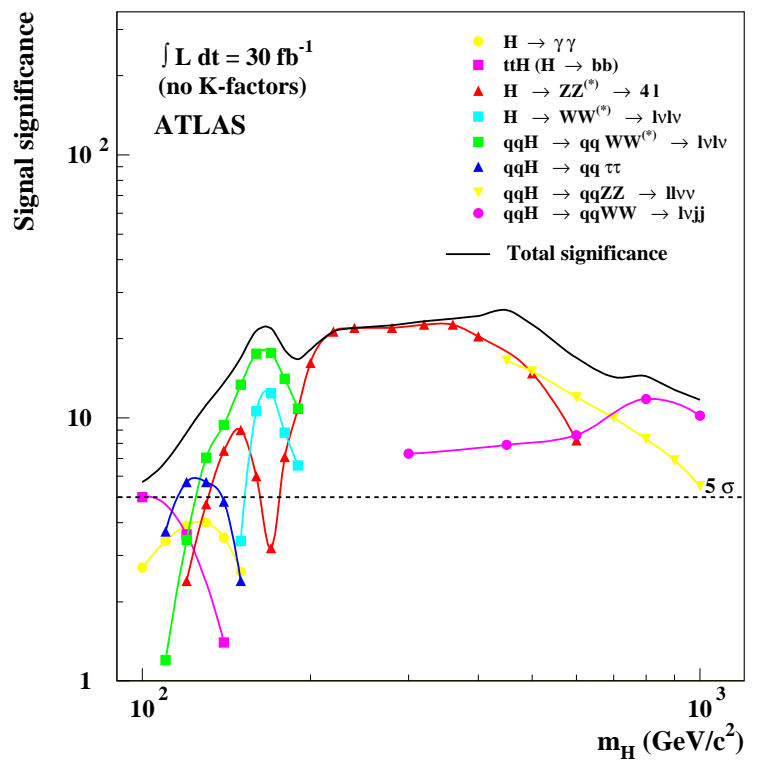

Figure 24: ATLAS sensitivity for the discovery of a Standard Model Higgs boson for an integrated luminosity of $30 \mathrm{fb}^{-1}$ over the full mass region. The signal significances are plotted for individual channels, as well as for the combination of channels. Systematic uncertainties on the background have been included for the

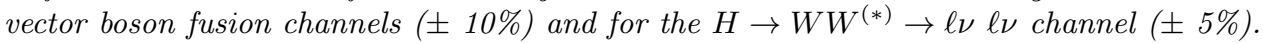

\subsection{3 $\quad H \rightarrow W W^{(*)}$ decays}

The signal significance in the $H \rightarrow W W^{(*)} \rightarrow \ell \nu \ell \nu$ decay mode can still be enhanced in the mass region around $160 \mathrm{GeV} / \mathrm{c}^{2}$ by searching for the associated production mode $W H$ with $W \rightarrow \ell \nu$, leading to a three-lepton final state accompanied by missing transverse momentum 103 The third lepton allows for a significant suppression of the background and therefore for a better signal-tobackground ratio than in the $g g \rightarrow H \rightarrow W W^{(*)}$ channel.

Furthermore, Higgs bosons decaying to $W W$ can be searched for in the associated $t \bar{t}$ production mode. Experimentally this channel leads to striking signatures with multilepton and multijet final states. In a recent study, final states with two like-sign leptons or with three leptons have been investigated 104 In order to reject the large backgrounds from heavy flavour decays into leptons, strict isolation criteria have been applied. After final cuts, the signal-to-background ratio is of the order of one for both channels and for the most favourable Higgs boson mass of $160 \mathrm{GeV} / \mathrm{c}^{2}$. Assuming an integrated luminosity of $30 \mathrm{fb}^{-1}$, about 21 and 13 signal events are expected for $m_{H}$ $=160 \mathrm{GeV} / \mathrm{c}^{2}$ in the two and in the three-lepton channel, respectively, compared to an expectation of about 20 background events from Standard Model processes in each channel. Significant contributions to the background arise from the associated $t \bar{t} W, t \bar{t} Z$ and $t \bar{t} t \bar{t}$ processes. It should be mentioned that the estimates of these backgrounds suffer from large uncertainties, and more studies are needed to obtain a more reliable estimate. For both smaller and larger Higgs boson masses, the signal significance decreases because of decreasing branching ratios and acceptance in the former and decreasing production cross section in the latter case 104 This channel provides important information for the determination of the Higgs boson couplings to top quarks, as discussed in Section 6.3.

\subsection{Combined signal significance}

The combined ATLAS Higgs boson discovery potential over the full mass range, $100<m_{H}<$ $1000 \mathrm{GeV} / \mathrm{c}^{2}$, assuming an integrated luminosity of $30 \mathrm{fb}^{-1}$ is shown in Fig. 24 The full mass range up to $\sim 1 \mathrm{TeV} / \mathrm{c}^{2}$ can be covered with a signal significance of more than $5 \sigma$ with several discovery channels available at the same time. In this evaluation no K-factors have been included. 


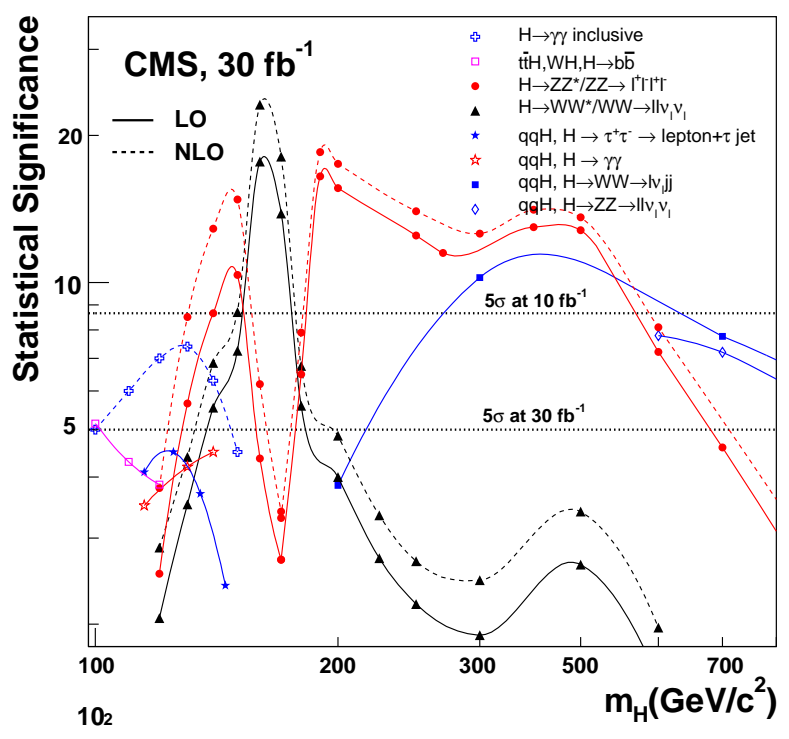

Figure 25: CMS sensitivity for the discovery of a Standard Model Higgs boson for an integrated luminosity of $30 \mathrm{fb}^{-1}$ over the full mass region. The signal significances are plotted for individual channels, as well as for the combination of channels. A systematic uncertainty of $\pm 5 \%$ on the background has been included for the $H \rightarrow W W^{(*)} \rightarrow \ell \nu \ell \nu$ channel. For the channels $H \rightarrow \gamma \gamma, H \rightarrow Z Z^{(*)} \rightarrow 4 \ell$ and $H \rightarrow W W^{(*)} \rightarrow \ell \nu \ell \nu$ results are shown for both leading order and next-to-leading order predictions for signals and backgrounds (from Ref. 69).

A similar performance has also been established for the CMS experiment $\underline{69}$ The corresponding discovery potential is shown in Fig. 25] It should be noted that for the $H \rightarrow \gamma \gamma, H \rightarrow Z Z^{(*)} \rightarrow 4 \ell$ and $H \rightarrow W W^{(*)} \rightarrow \ell \nu \ell \nu$ channels the analyses have also been performed by including NLO $\mathrm{K}$-factors for the signal and background processes. The results obtained are included in Fig. 25.

The various discovery channels available at the LHC are complementary both from physics and detector aspects. The different channels test three different production mechanisms, the gluon fusion, the vector boson fusion and the associated $t \bar{t} H$ production. This complementarity also provides sensitivity to non-standard Higgs models, such as fermiophobic models 105

Also from the experimental point of view the Higgs boson discovery potential at the LHC is robust. The searches exploiting the various production and decay modes are complementary in the sense that different detector components are important for different channels. The $H \rightarrow \gamma \gamma$ decays require excellent electromagnetic calorimetry. In the identification of vector boson fusion the measurement of jets, in particular the reconstruction of the forward tag jets, is essential. The Higgs detection in $b \bar{b}$ decays via the associated $t \bar{t} H$ production relies to a large extent on an excellent b-tagging performance.

\subsection{Outlook}

Given the recent progress in the calculation of higher order QCD corrections for both signal and background processes and their implementation in form of Monte Carlo programs, 10610739 activities have started to perform the experimental simulation using these new programs. In addition, new methods to match matrix element and parton shower calculations have been developed 108

In parallel, the simulation of the detector response is performed in more and more detail and the reconstruction algorithms are further developed and improved. It can therefore be expected that the uncertainties on the estimates of the signal significance will decrease in the near future.

In that context it is important to note that the steadily increasing data samples collected at the Tevatron allow to test the various background Monte Carlo predictions. This is an essential step to give confidence in background estimates for the LHC, such that a potential Higgs boson signature can be identified reliably. 


\section{Measurement of Higgs Boson Parameters at the LHC}

After a possible observation of a Higgs boson at the LHC it will be important to establish its nature. Besides a precise measurement of its mass, which enters electroweak precision tests, a determination of the spin and CP-quantum numbers is important. To establish that the Higgs mechanism is at work, measurements of the couplings of the Higgs boson to fermions and bosons as well as a demonstration of the Higgs boson self coupling are vital. The LHC potential for a measurement of these parameters is discussed in the following.

\subsection{Mass and width}

A precise measurement of the Higgs boson mass can be extracted from those channels where the invariant mass can be reconstructed from electromagnetic calorimeter objects, as in the case of $H \rightarrow \gamma \gamma$ and $H \rightarrow Z Z^{(*)} \rightarrow 4 \ell$ decays. $\left[8109\right.$ With an integrated luminosity of $300 \mathrm{fb}^{-1}$, each experiment would measure the Higgs boson mass with a precision of $\sim 0.1 \%$ over the mass range $100-$ $400 \mathrm{GeV} / \mathrm{c}^{2}$. The measurement error is determined by the absolute knowledge of the lepton energy scale, which is assumed to be $\pm 0.1 \%$. The precision could be slightly improved in the mass range between $\sim 150$ and $300 \mathrm{GeV} / \mathrm{c}^{2}$ if a scale uncertainty of $\pm 0.02 \%$ could be achieved. For larger masses, the precision deteriorates because the Higgs boson width becomes large and the statistical error increases. However, even for masses around $700 \mathrm{GeV} / \mathrm{c}^{2}$ a precision of about $1 \%$ can be reached.

The width of a Standard Model Higgs boson can be measured directly only for masses larger than about $200 \mathrm{GeV} / \mathrm{c}^{2}$, where the intrinsic width of the resonance is comparable to or larger than the experimental mass resolution. This is the mass region covered mainly by searches for $H \rightarrow Z Z \rightarrow 4 \ell$ decays. It has been estimated $\frac{68}{6}$ that over the mass range $300<m_{H}<700 \mathrm{GeV} / \mathrm{c}^{2}$ the precision of the measurement of the Higgs boson width is approximately constant and of the order of $6 \%$.

In the low mass region, $m_{H} \lesssim 200 \mathrm{GeV} / \mathrm{c}^{2}$, the Higgs boson width can be constrained only indirectly by using the visible decay modes, as proposed and discussed in Refs. 110 and 111

\subsection{Spin and CP eigenvalue}

After finding a resonance, one of the first priorities must be a determination of the spin and the $\mathrm{CP}$ eigenvalues. Recently, an analysis to determine these quantum numbers has been presented in Ref. [112. The decay mode $H \rightarrow Z Z \rightarrow 4 \ell$ has been used in the mass range above $200 \mathrm{GeV} / \mathrm{c}^{2}$ to extract information on the spin and $\mathrm{CP}$ eigenvalue by studying two distributions: (i) the distribution of the cosine of the polar angle $\cos \theta$ of the decay leptons relative to the $Z$ boson momentum and (ii) the distribution of the angle $\phi$ between the decay planes of the two $Z$ bosons in the rest frame of the Higgs boson. Since a heavy Higgs boson decays mainly into longitudinally polarized vector bosons, the cross section $d \sigma / d \cos \theta$ should show a maximum around $\cos \theta=0$. For a Standard Model Higgs boson a $(1+\beta \cos 2 \phi)$ like behaviour is expected for the angle $\phi$. However, the small vector coupling of leptons as well as experimental acceptance and resolution effects flatten this distribution. Apart from the Standard Model $\mathrm{CP}=0^{+}$Higgs boson, also a vector, pseudovector and a pseudoscalar scenario have been studied.

For Higgs boson masses above $250 \mathrm{GeV} / \mathrm{c}^{2}$, the distribution of the polar angle provides a good measurement of spin and CP. All scenarios considered can be separated from the Standard Model case with a significance of more than $8 \sigma$, assuming an integrated luminosity of $100 \mathrm{fb}^{-1}$. The decay plane angle correlation becomes more important for lower Higgs boson masses, where the discrimination power of the polar angle variable decreases and a determination of spin and $\mathrm{CP}$ is more difficult. However, with higher integrated luminosities non-Standard Model scenarios could still be ruled out, e.g., for $m_{H}=200 \mathrm{GeV} / \mathrm{c}^{2}$ the spin-1, CP-even hypothesis can be rejected with a significance of $6.4 \sigma$, while for the spin- 1 , CP-odd case, the significance is at the level of $3.9 \sigma$.

In Ref. 113 the method has been systematically generalized to arbitrary spin and parity assignments $\left(J^{P}\right)$ of the decaying particle. It has been shown that for $m_{H}>2 m_{Z}$ any odd spin state can be ruled out. Even spin states with $J>2$ may mimic the spin-0 case, but they could, nevertheless, be ruled out by measuring angular correlations of the $Z$ bosons with the initial state 113 Below the threshold for real ZZ production, i.e., for $H \rightarrow Z Z^{*}$ decays, the study of the threshold behaviour of the invariant mass spectrum of the off-shell $Z$ boson is used as a key element. The measurement of this distribution in combination with angular correlations allows to rule out non 
Table 1: List of all studies used in the global likelihood fit performed in Ref. 111, each channel has been used in the mass range indicated.

\begin{tabular}{l|l|c} 
Production mode & Decay mode & Mass range $\left(\mathrm{GeV} / \mathrm{c}^{2}\right)$ \\
\hline Gluon fusion & $H \rightarrow Z Z^{(*)} \rightarrow \ell \ell \ell \ell$ & $110-200$ \\
& $H \rightarrow W W^{(*)} \rightarrow \ell \nu \ell \nu$ & $110-200$ \\
& $H \rightarrow \gamma \gamma$ & $110-150$ \\
\hline Vector boson & $H \rightarrow Z Z^{(*)} \rightarrow \ell \ell \ell \ell$ & $110-200$ \\
fusion & $H \rightarrow W W^{(*)} \rightarrow \ell \nu \ell \nu$ & $110-190$ \\
& $H \rightarrow \tau \tau \rightarrow \ell \nu \nu \ell \nu \nu$ & $110-150$ \\
& $H \rightarrow \tau \tau \rightarrow \ell \nu \nu h a d \nu$ & $110-150$ \\
& $H \rightarrow \gamma \gamma$ & $110-150$ \\
\hline$t \bar{t}$ H production & $H \rightarrow W W^{(*)} \rightarrow \ell \nu \ell \nu(\ell \nu)$ & $120-200$ \\
& $H \rightarrow b \bar{b}$ & $110-140$ \\
& $H \rightarrow \gamma \gamma$ & $110-120$ \\
\hline$W H$ production & $H \rightarrow W W^{(*)} \rightarrow \ell \nu \ell \nu(\ell \nu)$ & $150-190$ \\
& $H \rightarrow \gamma \gamma$ & $110-120$ \\
$Z H$ production & $H \rightarrow \gamma \gamma$ & $110-120$ \\
\hline
\end{tabular}

Standard Model $J^{P}$ assignments. However, a full experimental simulation, including backgrounds and detector resolution effects, still needs to be carried out.

In this context it should be noted that the $J=1$ hypothesis could also be ruled out by observing non-zero $H \gamma \gamma$ and $H g g$ couplings.

As suggested in Ref. 114, the $t \bar{t} H$ production channel could potentially be used to distinguish a $\mathrm{CP}$-even from a CP-odd Higgs boson. The method proposed requires the reconstruction of the momenta of both top quarks. The corresponding studies by the LHC collaborations have not yet been made.

Higgs boson production via weak boson fusion can be used to determine the CP properties via the

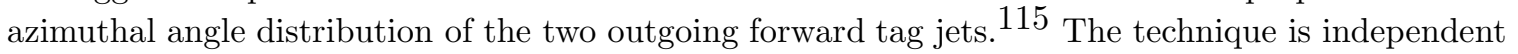
of the Higgs boson mass and of the observed decay channel.

\subsection{Couplings to bosons and fermions}

For a Higgs boson with $m_{H}<2 m_{Z}$, the experiments at the LHC will be able to observe the dominant decays into bosons and heavy fermions over mass ranges where the branching ratios in question are not too small. Decays to light fermions, i.e., to electrons, muons or to quarks lighter than the b-quark, will, however, not be observable either due to the small decay rate or due to the overwhelming background from QCD jet production.

In that mass range, the total width is expected to be small enough such that the narrow width approximation can be used to extract the couplings. The rates $\sigma \cdot B R(H \rightarrow f \bar{f})$ measured for final states $f \bar{f}$ are to a good approximation proportional to $\Gamma_{i} \cdot \Gamma_{f} / \Gamma$, where $\Gamma_{i}$ and $\Gamma_{f}$ are the Higgs boson partial widths involving the couplings at production and decay, respectively, and $\Gamma$ is the total width of the Higgs boson.

The strength of the LHC in the coupling measurements is based on the simultaneous information which, for a given Higgs boson mass, is available in the various production and decay modes. Recently, a study has been performed where the full information of all accessible production and decay channels, as listed in Table 1 is used to fit the coupling parameters 111 In this study, the correlations among the various channels as well as experimental and theoretical systematic uncertainties are taken into account.

Assuming that the measured values correspond to the Standard Model expectations, a likelihood function is formed which, for a given integrated luminosity, is based on the expected Poisson distribution of the event numbers and on the estimated systematic errors. These errors include a $5 \%$ uncertainty on the integrated luminosity, uncertainties on the reconstruction and identification efficiencies of leptons (2\%), photons (2\%), b-quarks (3\%) and on the forward jet tagging and jet veto efficiency (5\%). In addition, theoretical uncertainties on Higgs boson production ( $20 \%$ for $g g H$, $15 \%$ for $t \bar{t} H, 7 \%$ for $W H$ and $Z H$ and $4 \%$ for vector boson fusion) and on branching fractions (1\%) have been taken into account. 

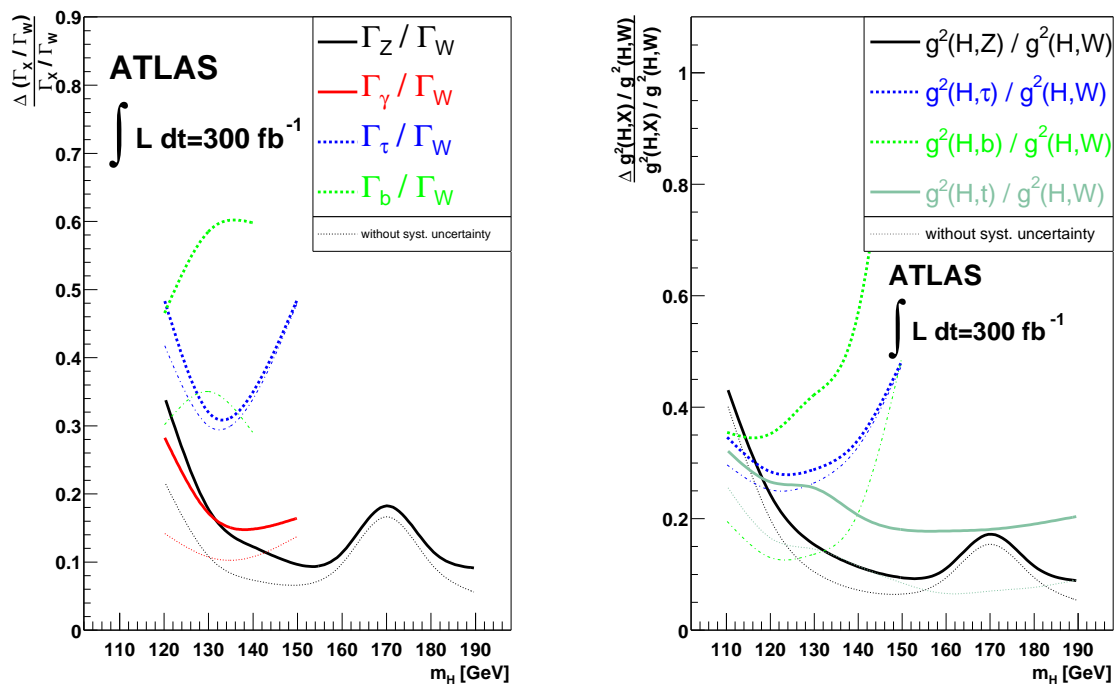

Figure 26: The expected relative errors for the measurement of relative branching ratios (left) and relative couplings (right), normalized to those of the $H \rightarrow W W$ decay and assuming an integrated luminosity of $300 \mathrm{fb}^{-1}$ for one experiment. The dashed lines give the expected relative error without systematic uncertainties (from Ref. 111).

Under the assumption that only one scalar CP-even Higgs boson exists, relative branching ratios, which are identical to ratios of partial decay widths, can be measured. The decay $H \rightarrow W W$ is used as normalization since it can be measured over the full intermediate mass range with a relatively small error. In Figure 26 (left) the expected relative errors on the measurement of ratios of Higgs boson branching ratios are shown, assuming an integrated luminosity of $300 \mathrm{fb}^{-1}$. In particular the ratios $\Gamma_{Z} / \Gamma_{W}$ can be measured with an accuracy of the order of $10-20 \%$ for Higgs boson masses above $130 \mathrm{GeV} / \mathrm{c}^{2}$. The ratios $\Gamma_{\tau} / \Gamma_{W}$ and $\Gamma_{b} / \Gamma_{W}$ are less constrained, with errors expected to be of the order of 30 to $60 \%$. It should be noted that these results are based on information from the decay only and do not exploit any information from the production.

If additional theoretical assumptions are made, information from the production can be used as well, and in particular a measurement of the top-Yukawa coupling becomes possible via the strong dependence of the gluon fusion and $t \bar{t} H$ production cross sections on this coupling. Assuming that only the known particles of the Standard Model couple to the Higgs boson and that no couplings to light fermions are extremely enhanced, all accessible Higgs boson production and decay modes at the LHC can be expressed in terms of the Higgs boson couplings $g_{W}, g_{Z}, g_{t}, g_{b}$ and $g_{\tau}$. The production cross sections depend on the square of these couplings. The exact dependence has to be calculated theoretically and put into the fit with corresponding systematic uncertainties. The gluon fusion production cross section, for example, is not strictly proportional to the top-Yukawa coupling squared, but receives additional contributions from the interference with diagrams containing a bloop. Using the program of Ref. 116, they have been found to be at the level of $7 \%$ for $m_{H}=$ $110 \mathrm{GeV} / \mathrm{c}^{2}$ and $4 \%$ for $m_{H}=190 \mathrm{GeV} / \mathrm{c}^{2}$. These additional contributions are ignored and it is assumed that the $b$-coupling is not enhanced by a factor of 10 or more compared to the Standard Model. Furthermore, the interference effect between $W$-and $Z$-fusion in the vector boson fusion is expected to be small $(<1 \%)^{19}$ and is ignored.

Similarly, the Higgs boson branching ratios are proportional to $g^{2} / \Gamma$, and again, the proportionality factors are taken from theory, assuming a relative uncertainty of about $1 \%$. The decay $H \rightarrow \gamma \gamma$ proceeds either via a $W$ or a top-quark loop, with destructive interference between the two. The relative contributions are taken from Ref. 117 .

In Figure 26(right) the relative errors on the measurement of relative couplings are shown for an integrated luminosity of $300 \mathrm{fb}^{-1}$ for one experiment. Due to the large contributions of the gluonfusion and $t \bar{t} H$ production modes, the ratio of the top-Yukawa coupling to the Higgs boson coupling to $W$ bosons can be well constrained with an estimated uncertainty of the order of 10 to $20 \%$.

Recently, the determination of Higgs boson couplings at the LHC has been discussed for gen- 
eral multi-Higgs doublet models $\frac{118}{11}$ Imposing the additional constraint that the $H W W$ and $H Z Z$ couplings are bound from above by their Standard Model values $\left(g_{W / Z}^{2}(S M)\right)$, i.e., $g_{W / Z}^{2}<1.05$. $g_{W / Z}^{2}(S M)$, an absolute measurement of the Higgs boson couplings to the vector bosons and the $\tau$ lepton, $b$ and $t$ quark becomes possible. The constraints imposed are theoretically motivated and are valid in particular for the MSSM. Any model that contains only Higgs doublets and/or singlets satisfies the relation $g_{W / Z}^{2}<g_{W / Z}^{2}(S M)$. The extra $5 \%$ margin allows for theoretical uncertainties in the translation between couplings-squared and partial widths and also for small admixtures of exotic Higgs states, as for example $\mathrm{SU}(2)$ triplets. 118 Contributions of additional particles running in the loops for $H \rightarrow \gamma \gamma$ and $g g \rightarrow H$ are allowed for and their contribution to the partial width is fitted. Assuming an integrated luminosity of $300 \mathrm{fb}^{-1}$ (for all channels, except vector boson fusion, for which $100 \mathrm{fb}^{-1}$ have been assumed) and a combination of two experiments, typical accuracies for the absolute measurement of the couplings of about 20 to $30 \%$ can be achieved for Higgs boson masses below $160 \mathrm{GeV} / \mathrm{c}^{2}$. For masses above the $W$-pair threshold the measurement of the $W$ and $Z$ partial width can be performed with an accuracy at the level of $\pm 10 \%$.

\subsection{Higgs boson self coupling}

To fully establish the Higgs mechanism, it must be demonstrated that the shape of the Higgs potential $V_{H}$ has the form required for electroweak symmetry breaking. The potential can be expressed in terms of the physical Higgs field $H$ as $V_{H}=\frac{m_{H}^{2}}{2} H^{2}+\frac{m_{H}^{2}}{2 v} H^{3}+\frac{m_{H}^{2}}{8 v^{2}} H^{4}$, where $v=$ $\left(\sqrt{2} G_{F}\right)^{-1 / 2}=246 \mathrm{GeV} / \mathrm{c}^{2}$ is the Higgs vacuum expectation value. The coefficients of the second and third term are proportional to the strength of the Higgs trilinear $\left(\lambda_{H H H}=3 m_{H}^{2} / v\right)$ and quartic $\left(\lambda_{H H H H}^{\prime}=3 m_{H}^{2} / v^{2}\right)$ Higgs boson self coupling, respectively. In order to extract information on these couplings, multiple Higgs boson production must be measured ${ }^{[119}$ Since the quartic coupling is about two orders of magnitude smaller than the trilinear coupling, present studies have focussed on the determination of $\lambda_{H H H}$.

The coupling strength $\lambda_{H H H}$ enters the production rate of Higgs boson pairs. At LHC energies, the inclusive Higgs boson pair production is dominated by gluon fusion 120 Other processes have cross sections which are factors of 10 to 30 smaller. For $m_{H}>140 \mathrm{GeV} / \mathrm{c}^{2}, H \rightarrow W W$ is the dominant decay mode and $W W W W$ final states are produced with the largest branching ratio. In order to suppress the large backgrounds from $t \bar{t}$ and multiple gauge boson production, like-sign lepton final states offer the best sensitivity 121122123 Studies have shown that the $\left(\ell^{ \pm} \nu j j\right)\left(\ell^{ \pm} \nu j j\right)$ final state has the highest sensitivity for extracting information on the self coupling parameter $\lambda_{H H H}$.

The studies conclude that with data corresponding to the ultimate luminosity expected at the LHC of $300 \mathrm{fb}^{-1}$ per experiment, a determination of the Higgs boson self coupling will not be possible. A measurement with reasonable errors will require a luminosity upgrade, i.e., the realization of the so called Super $L H C$ (SLHC).

In the analysis of Ref. 122 it is concluded that the LHC may rule out the case of a non-vanishing $\lambda_{H H H}$ using the four $W$ final state for a mass range of $150<m_{H}<200 \mathrm{GeV} / \mathrm{c}^{2}$ with a confidence level of $95 \%$. For the SLHC, assuming an integrated luminosity of $3000 \mathrm{fb}^{-1}$ per experiment, it is claimed that a measurement of $\lambda_{H H H}$ with a precision of $20 \%$ will be possible. In this study NLO $\mathrm{K}$-factors have been used.

These conclusions still need to be confirmed in a full simulation of the detector performance. First preliminary studies $\frac{123}{2}$ confirm the sensitivity at the SLHC, however, some background contributions might have been underestimated. Further studies to clarify these issues are currently in progress.

The mass region $m_{H} \lesssim 140 \mathrm{GeV} / \mathrm{c}^{2}$ is considered to be very challenging for a measurement of the Higgs boson self coupling, even at the SLHC ${ }^{122}$ Recently, it has been proposed to use the rare decay mode $H H \rightarrow b \bar{b} \gamma \gamma$ to investigate the self coupling in that mass region.124] At the LHC, assuming an integrated luminosity of $300 \mathrm{fb}^{-1}$ per experiment and including NLO K-factors, only six signal events are expected for the most optimistic case of $m_{H}=120 \mathrm{GeV} / \mathrm{c}^{2}$. Using this decay mode would require a luminosity upgrade to rule out $\lambda_{H H H}=0$ at the $90 \%$ confidence level 124 Also in this case, a detailed simulation of the detector performance has not yet been completed. 


\section{Search for MSSM Higgs Bosons at the LHC}

In addition to the excellent prospects for Standard Model Higgs searches, the LHC experiments have a large potential in the investigation of the MSSM Higgs sector. The experimental searches carried out at LEP and presently continued at the Tevatron can be extended to much larger Higgs boson masses. In the search for the light, Standard Model-like Higgs boson $h$ the same channels as in the search for the Standard Model Higgs boson will be used. Heavier Higgs bosons will be searched for in additional decay channels which become accessible in certain regions of the MSSM parameter space due to enhanced couplings, e.g., the decay mode $H / A \rightarrow \tau \tau$ at large $\tan \beta$. Decays into $\tau$ leptons also contribute to the search for charged Higgs bosons, which at the LHC can be extended to masses beyond the top-quark-mass. A comprehensive and complete study of the LHC discovery potential in the MSSM has first been presented in Ref. [125], In that study, the discovery potential had been determined for two benchmark scenarios with different assumptions on mixing in the stop sector, as defined at LEP ${ }^{[126]}$ In a so-called no-mixing scenario the trilinear coupling in the stop sector $A_{t}$ is set to zero, whereas in the so-called maximal mixing scenario the value $A_{t}=\sqrt{6} m_{S U S Y}$, with $m_{S U S Y}=1 \mathrm{TeV} / \mathrm{c}^{2}$, is used. In the following, the search strategies for MSSM Higgs bosons at the LHC are briefly discussed and the discovery potential is presented for different MSSM benchmark scenarios. Throughout this chapter, all discovery contours for MSSM Higgs bosons in the $\left(m_{A}, \tan \beta\right)$-parameter space are given for a signal significance at the $5 \sigma$ level. In most analyses SUSY particles are considered to be heavy enough to play a negligible rôle in the phenomenology of Higgs boson decays. This assumption is abandoned in Section [7.5 where the interplay of the Higgs sector and SUSY particles is discussed.

\subsection{Search for the light CP-even Higgs boson $h$}

The channels discussed in Section [ 5 can be used to search for the lightest MSSM CP-even Higgs boson $h$. Results for the discovery potential obtained in studies carried out by the CMS collaboration are shown in the $\left(m_{A}, \tan \beta\right)$-plane in Fig. 27. In these studies, maximal stop mixing, a top-quark mass of $175 \mathrm{GeV} / \mathrm{c}^{2}, \mathrm{~m}_{S U S Y}=1 \mathrm{TeV} / \mathrm{c}^{2}$ and integrated luminosities of 30,60 and $100 \mathrm{fb}^{-1}$ have been assumed 127 In addition to the $H \rightarrow \gamma \gamma, H \rightarrow Z Z^{(*)} \rightarrow 4 \ell$ and $t t H$ with $H \rightarrow b \bar{b}$ channels the vector boson fusion channel $q q H \rightarrow q q \tau \tau$ contributes significantly to the discovery potential. For an integrated luminosity of $100 \mathrm{fb}^{-1}$ the whole parameter space, except the region $90<m_{A}<$ $130 \mathrm{GeV} / \mathrm{c}^{2}$, can be covered with a significance of more than $5 \sigma$ by a single experiment.

In the maximal mixing scenario, the Higgs bosons $h$ and $A$ are nearly degenerate in mass in the uncovered region up to $m_{A} \sim 125 \mathrm{GeV} / \mathrm{c}^{2}$. At large $\tan \beta$ the enhanced coupling to $b$ quarks can be used to search for these Higgs bosons in the $p p \rightarrow b \bar{b} h / A \rightarrow b \bar{b} \mu \mu$ channel. It has been shown that signals can be extracted with a significance above $5 \sigma$ for $105<m_{A}<125 \mathrm{GeV} / \mathrm{c}^{2}$ and $\tan \beta \gtrsim 15128129$ The signal extraction is challenging due to the overwhelming background from $Z \rightarrow \mu \mu$ production. It will therefore be essential to have a good b-tagging performance and a good dimuon mass resolution as well as to control the background from data, using $Z \rightarrow \ell \ell$ control samples. It should be noted that this particular part of the parameter space can also be covered with a significance exceeding $5 \sigma$ by searches for charged Higgs bosons (see Sections 7.3 and 7.4 ).

\subsection{Search for the heavy MSSM Higgs bosons $\mathrm{H}$ and A}

\subsubsection{The large $\tan \beta$ region}

At large $\tan \beta$ where the couplings of the heavy Higgs bosons $H$ and $A$ to down-type fermions are enhanced, the associated Higgs boson production with a $b \bar{b}$ pair is the dominant production mode. The decay into $\tau$ pairs has a significant branching ratio over a large region of parameter space, such that the process $b \bar{b} H / A \rightarrow b \bar{b} \tau \tau$ plays a key role. This channel can be complemented by the $H / A \rightarrow \mu \mu$ decay mode, for which the much smaller branching ratio is compensated by the better signal-to-background ratio. In addition, a search in the $b \bar{b} b \bar{b}$ final state has been proposed in the literature 130 but it suffers from sizeable background from QCD jet production and therefore has only a limited discovery potential 68

For the $b \bar{b} \tau \tau$ final states both excellent b-tagging and tau identification performance are essential to suppress the large backgrounds from $\gamma^{*} / Z$, QCD multijet and $W+$ jet production. Detailed studies 

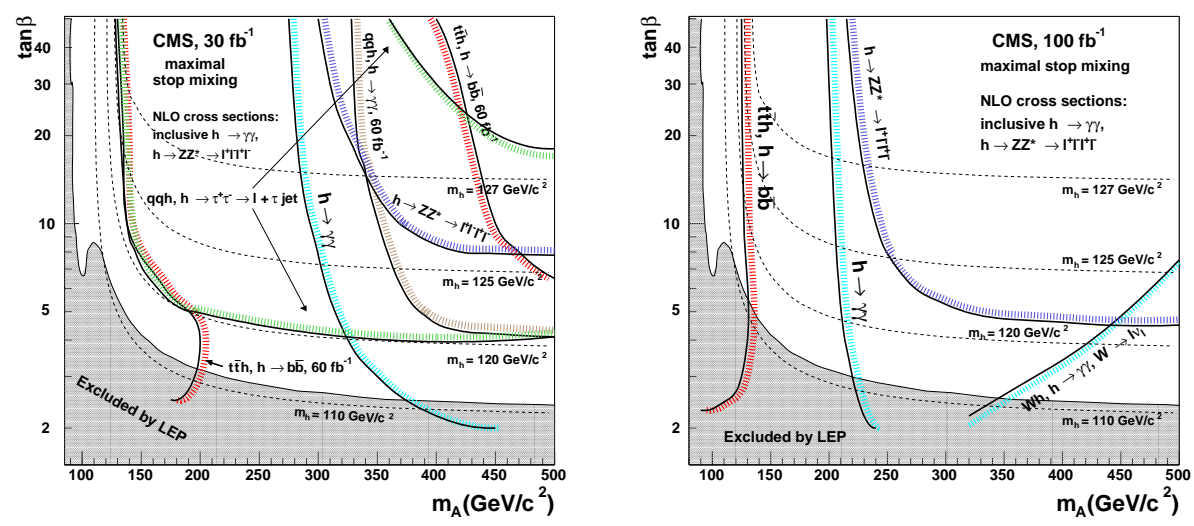

Figure 27: (Left): The $5 \sigma$ discovery potential for the light CP-even MSSM-Higgs boson $h$ in the $\left(m_{A}\right.$, tan $\left.\beta\right)$-plane assuming maximal stop mixing. Integrated luminosities of 30 and $60 \mathrm{fb}^{-1}$ are assumed for the gluon-fusion channels $\left(H \rightarrow \gamma \gamma\right.$ and $\left.H \rightarrow Z Z^{(*)} \rightarrow 4 \ell\right)$ and for the associated $t \bar{t} h$ and vector boson fusion channels (qqh), respectively. For the gluon-fusion channels NLO cross sections are used. (Right): The same, assuming an integrated luminosity of $100 \mathrm{fb}^{-1}$ for all channels (from Ref. 69).

have shown that both leptonic and hadronic tau decays $\left(\tau_{\text {had }}\right)$ can be used to isolate signals from heavy Higgs boson production. ${ }^{131132}$ For $m_{A} \lesssim 400 \mathrm{GeV} / \mathrm{c}^{2}$ the final state with one leptonic and one hadronic tau decay $\left(\ell-\tau_{h a d}\right)$ has the largest discovery reach. At large $m_{A}$ the double hadronic decay mode contributes significantly, while for small Higgs boson masses its reach is limited by the low trigger efficiency. In all decay modes the $\tau \tau$ invariant mass can be reconstructed using the collinear approximation, as discussed in Section 5.2 An example of a reconstructed $H / A$ signal with $m_{H / A}=500 \mathrm{GeV} / \mathrm{c}^{2}$, based on a simulation of the CMS detector performance in the $\tau_{\text {had }}-\tau_{\text {had }}$ decay mode, is shown in Fig. 28. An integrated luminosity of $60 \mathrm{fb}^{-1}$ and $\tan \beta=30$ have been assumed. The discovery contours for the various $\tau \tau$ decay modes are shown in the $\left(m_{A}, \tan \beta\right)$ plane in Fig. 29 for the combination of both LHC experiments assuming an integrated luminosity of $30 \mathrm{fb}^{-1}$.

As mentioned above, also the $\mu \mu$ decay mode contributes to the discovery potential. Given the good mass resolution for dimuon final states, this channel can eventually be used to separate nearby Higgs boson resonances using a precise measurement of the shape of the mass distribution. For small $m_{A}$ it covers regions of parameter space not yet excluded by the LEP experiments (see discussion at the end of Section 7.1 .

\subsubsection{The small $\tan \beta$ region}

In the region of small tan $\beta$ heavy Higgs bosons can be searched for via their decays into the lightest Higgs boson. This allows for a simultaneous observation of two Higgs bosons, for example via the decay modes $H \rightarrow h h$ and $A \rightarrow Z h$.

The final states of interest for a search in the $H \rightarrow h h$ decay mode are $b \bar{b} \gamma \gamma, b \bar{b} \tau \tau$ and $b \bar{b} b \bar{b}$. The decays into $b \bar{b} \gamma \gamma$ can reliably be triggered on and offer good kinematic constraints and mass resolution for the reconstruction of $m_{H}$. However, due to the small branching ratio into $\gamma \gamma$, the discovery potential in that channel is limited by the small signal rate. For an integrated luminosity of $300 \mathrm{fb}^{-1}$, an obervation is possible in the parameter region $\tan \beta<4$ and $2 m_{h}<m_{H}<2 m_{t}$. 68 The $5 \sigma$ discovery contour is shown in Fig. 29] assuming an integrated luminosity of $30 \mathrm{fb}^{-1} \mathrm{for}^{\mathrm{each}}$ LHC experiment.

Searches in both the $b \bar{b} \tau \tau$ and $b \bar{b} b \bar{b}$ final states have been found to be less promising! ${ }^{68}$ For the $b \bar{b} \tau \tau$ case, the signal extraction is difficult due to large backgrounds from $W+$ jets and $t \bar{t}$ production and due to the poor mass resolution for the signal. A detection of the $H \rightarrow h h$ decay in the $b \bar{b}$ $b \bar{b}$ mode requires a four-jet trigger with as low a $P_{T}$ threshold as possible and excellent b-tagging performance to control the overwhelming background from four-jet events. 


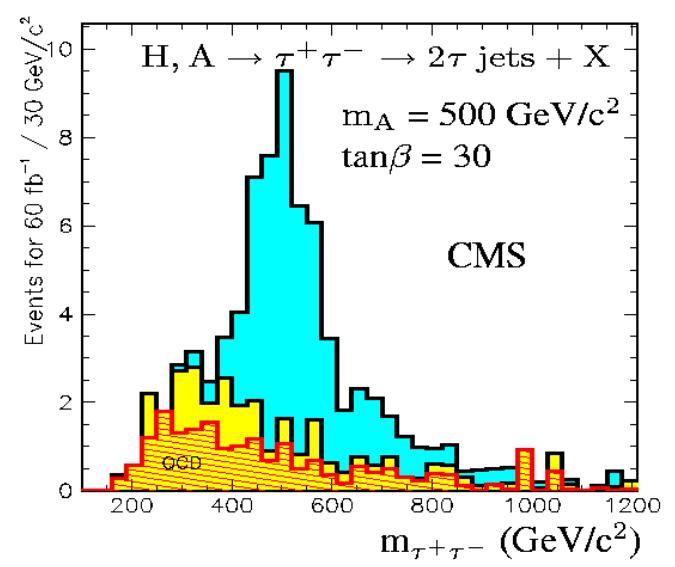

Figure 28: The reconstructed $\tau \tau$ invariant mass distribution in the fully hadronic final state $\left(\tau_{\text {had }}-\right.$ $\tau_{\text {had }}$ ) from the process $g g \rightarrow b \bar{b} H / A \rightarrow b \bar{b} \tau \tau$. The $H / A$ signal (dark) for $m_{A}=500$ GeV $/ c^{2}$ and $\tan \beta=30$ is shown on top of the background for an integrated luminosity of $60 \mathrm{fb}^{-1}$ (from Ref. 69).

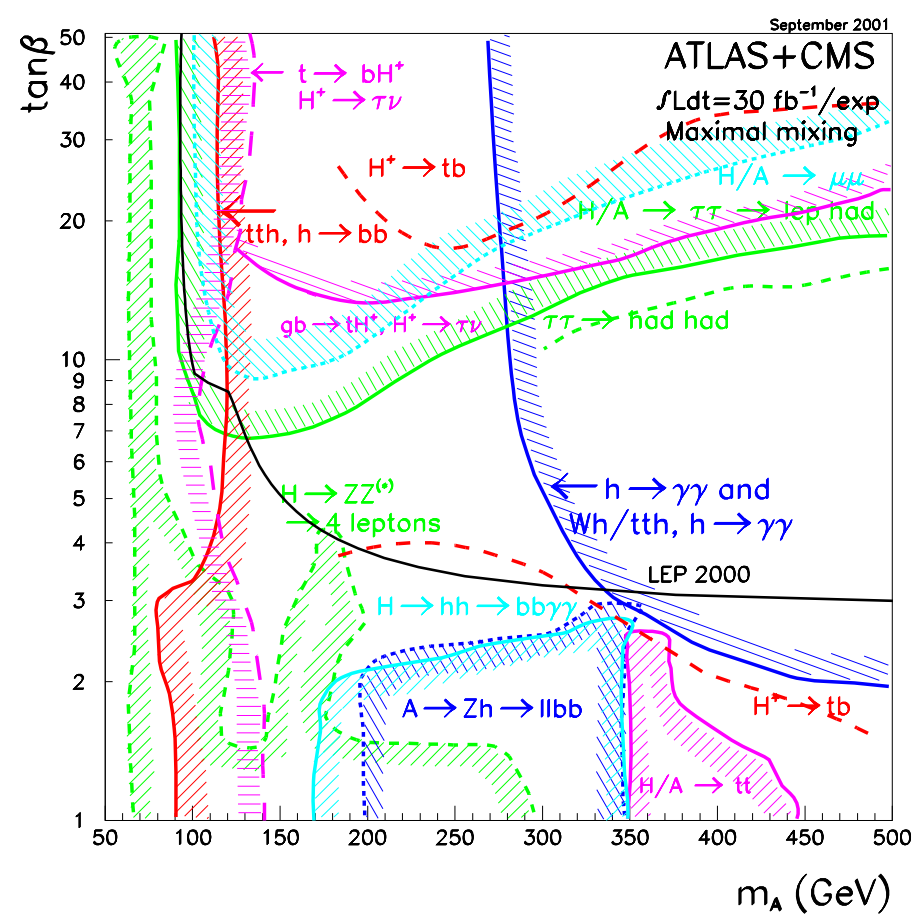

Figure 29: The combined sensitivity of the ATLAS and CMS experiments for the discovery of MSSM Higgs bosons in the maximal mixing scenario for an integrated luminosity of $30 \mathrm{fb}^{-1}$. The $5 \sigma$ discovery contour curves are shown in the $\left(m_{A}\right.$, tan $\left.\beta\right)$-plane for individual channels not including the vector boson fusion channels. The limit from the LEP experiments is superimposed (from Ref. 133). 
The $A \rightarrow Z h$ decay mode has so far been studied in the final states $Z h \rightarrow \ell \ell b \bar{b}$ and $Z h \rightarrow$ $b \bar{b} b \bar{b} .6869$ A search in the latter decay mode is as challenging as the $H \rightarrow h h \rightarrow b \bar{b} b \bar{b}$ channel discussed above. The decays into $\ell \ell b \bar{b}$ can easily be triggered on and offer the largest rates apart from the dominant $b \bar{b} b \bar{b}$ decays. Given that the signal rate is rapidly falling with increasing $\tan \beta$, the $A \rightarrow Z h \rightarrow \ell \ell b \bar{b}$ channel can only be observed at small $\tan \beta$ and for $200 \mathrm{GeV} / \mathrm{c}^{2}<m_{A}<2 m_{t}$. The $5 \sigma$ discovery contour is shown in Fig. 29 assuming an integrated luminosity of $30 \mathrm{fb}^{-1}$ for each LHC experiment.

For $m_{A}>2 m_{t}$ and $\tan \beta \sim 1$, the $H \rightarrow t \bar{t}$ and $A \rightarrow t \bar{t}$ branching ratios are close to $100 \%$. Since the $H$ and $A$ bosons are almost degenerate in mass in the relevant region of parameter space, the two decays cannot be distinguished experimentally. As discussed in the literature, 134 a signal from $H / A \rightarrow t \bar{t}$ would appear as a peak in the $t \bar{t}$ invariant mass spectrum above the $t \bar{t}$ continuum background. The interference of the signal and background amplitudes leads to a strong suppression of the signal at high masses. For a Higgs boson mass of $370(450) \mathrm{GeV} / \mathrm{c}^{2}$, the signal-to-background ratio is expected to be of the order of $9 \%(1 \%)$ only. Under the optimistic assumption that the background $t \bar{t}$ mass spectrum will be known to better than $1 \%$ from experimental data and theory, a signal extraction would be possible in a limited region of parameter space for $2 m_{t}<m_{A}<$ $450 \mathrm{GeV} / \mathrm{c}^{2}$. The $5 \sigma$ discovery contours are shown in Fig. 29]

\subsection{Charged Higgs bosons}

At the LHC, a charged Higgs boson can be detected in several scenarios. For $m_{H^{ \pm}}<m_{t}-m_{b}$, top-quark production represents a copious source via the decay $t \rightarrow H^{ \pm} b$. If the charged Higgs boson is heavier than the top quark, it can be produced via the process $g g \rightarrow H^{ \pm} t b\left(\right.$ or $\left.g b \rightarrow H^{ \pm} t\right) \underline{1351136}$ Additional contributions come from the Drell-Yan type process $g g, q \bar{q} \rightarrow H^{+} H^{-}$and from the associated production with a $W$ boson, $q \bar{q} \rightarrow H^{ \pm} W^{\mp} 137$ Assuming a heavy SUSY mass spectrum, the charged Higgs boson decays into Standard Model particles only. For small $\tan \beta$ and $m_{H^{ \pm}}<m_{t}$, the main decay channels are $H^{ \pm} \rightarrow \tau \nu, c s, W h$ and $t^{*} b$. For mass values above the top-quark mass, the $H^{ \pm} \rightarrow t b$ decay mode becomes dominant, in particular at small $\tan \beta$. At larger $\tan \beta$, also the $H^{ \pm} \rightarrow \tau \nu$ decay has a significant branching ratio, which, however, decreases with increasing Higgs boson mass (see Fig. [6 for details).

Due to the large $t \bar{t}$ production cross section, charged Higgs bosons with $m_{H^{ \pm}}<m_{t}-m_{b}$ can be detected nearly up to the kinematic limit. The LHC discovery potential has been studied for both the dominant $H^{ \pm} \rightarrow \tau \nu$ and the $H^{ \pm} \rightarrow q q^{\prime}$ channel, via the decay chain $p p \rightarrow t \bar{t} \rightarrow H^{ \pm} b W^{\mp} \bar{b} .6869$ In the region $5<\tan \beta<10$, the hadronic decay mode contributes significantly and increases the discovery potential! 138

Following the studies presented in Refs. 139 and 140 both LHC collaborations have recently evaluated their discovery potential for heavier charged Higgs bosons, i.e., $m_{H^{ \pm}}>m_{t}-m_{b}$. 141142 For the process $g b \rightarrow H^{ \pm} t \rightarrow t b t \rightarrow \ell \nu b b q \bar{q} b$, the large backgrounds can be sufficiently suppressed if three b-tags are required and both top quarks are reconstructed. In addition, it has been studied whether the discovery range in the $H^{ \pm} \rightarrow t b$ decay mode can be extended if four b-jets are required in the final state (via the process $g g \rightarrow H^{ \pm} t b$ ) 143 Although this requirement reduces the background, the reconstruction of the charged Higgs boson is rendered more difficult due to the combinatorics in the reconstruction of the multijet final state. Overall, this channel does not lead to a significant extension of the discovery potential provided by the three b-tag analysis.

More favourable signal-to-background conditions are found in the $H^{ \pm} \rightarrow \tau \nu$ decay mode for the production process $g b \rightarrow H^{ \pm} t \rightarrow \tau \nu q q b$, with $\tau \rightarrow h a d \nu$. Both the associated top quark and the tau lepton are required to decay hadronically. This has the advantage that the transverse mass of the $\tau_{\text {had }}-P_{T}$ system can be used to discriminate between signal and background. The signal distribution is expected to show a Jacobian peak structure at the Higgs boson mass. The channel must be triggered by a hadronic tau $+P_{T}$ or hadronic tau + multijet trigger, both of which are foreseen in the two LHC experiments. The backgrounds from $W+$ jet, $t \bar{t}, W t$ and QCD multijet production can be suppressed by requiring in addition to an identified hadronic tau exactly three high- $P_{T}$ jets, one of which must be b-tagged. The signal can be enhanced by exploiting the $\tau$ polarization in one prong tau decays $\frac{140}{14}$ which leads to a harder spectrum of single pions when the tau originates from an $H^{ \pm}$rather than from a $W$ decay. Requiring, e.g., that more than $80 \%$ of the visible tau energy is carried by the single charged pion, the $t \bar{t}$ background can be reduced by a 


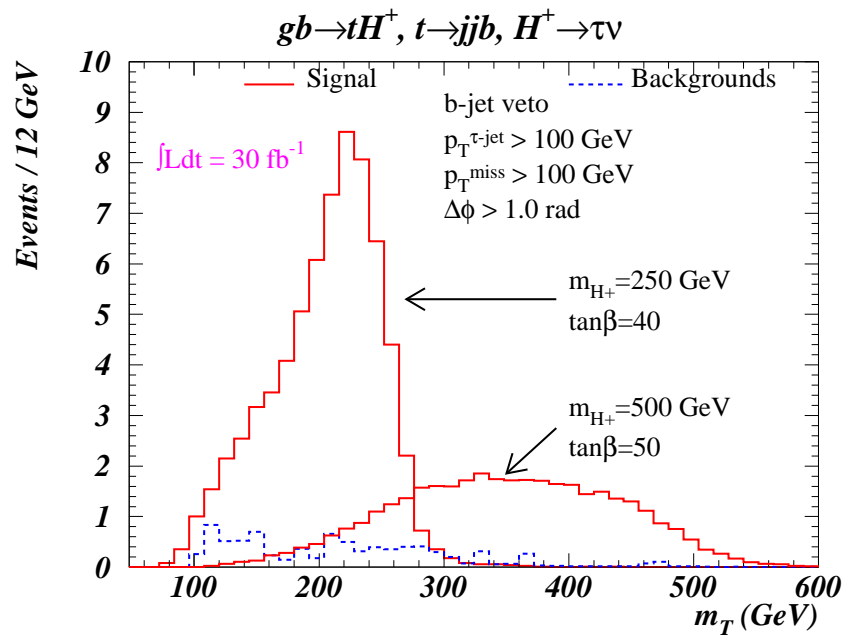

Figure 30: The reconstructed transverse mass of the $\tau_{\text {had }}-\not P_{T}$ system from the process $g b \rightarrow H^{+} t \rightarrow$ $\tau \nu$ qqb for charged Higgs bosons with masses of $250 \mathrm{GeV} / \mathrm{c}^{2}$ (at $\left.\tan \beta=40\right)$ and $500 \mathrm{GeV} / \mathrm{c}^{2}$ (at $\tan \beta=50)$ in comparison to the background. The simulation has been performed for the ATLAS experiment assuming an integrated luminosity of $30 \mathrm{fb}^{-1}$ (from Ref. 141).

factor of $\sim 300$ while the signal efficiency can be kept at the $10-20 \%$ level 142 The discrimination between signal and background is shown in Fig. 30] where the reconstructed transverse mass of the $\tau_{\text {had }}-\not_{T}$ system is shown for two Higgs boson masses in comparison to the background. After a transverse mass cut, the backgrounds are small and a charged Higgs boson can be discovered with a significance of more than $5 \sigma$ for $m_{H^{ \pm}}>m_{t}$ in the large $\tan \beta$ region. In the $\tau \nu$ decay mode, the two b-tag final state (via $g g \rightarrow H^{ \pm} t b$ production) also contributes to the discovery potential 127

The discovery contours for both the $H^{ \pm} \rightarrow \tau \nu$ and the $H^{ \pm} \rightarrow t b$ channels are superimposed in the $\left(m_{A}, \tan \beta\right)$-plane in Fig. 29] Recently, the discovery potential in the transition region $\left(m_{H^{ \pm}} \sim m_{t}\right)$ has been studied more carefully $\frac{144}{1 n}$ particular, it has been shown that charged Higgs bosons can be detected over the full transition region using the $H^{ \pm} \rightarrow \tau \nu$ decay mode.

Within the MSSM, the sensitivity to the $H^{ \pm} \rightarrow W^{(*)} h$ decay mode is weaker 141 For Higgs boson masses below the top-quark mass, the extraction of the signal is only possible in the small $\tan \beta$ region $(1.5<\tan \beta<2.5)$. For Higgs boson masses above the top-quark mass, the discovery potential in this channel is marginal. However, in extended models, as for example in the NMSSM 145 where the Higgs sector of the MSSM is extended by a complex singlet scalar field, the $H^{ \pm} \rightarrow W^{(*)} h$ channel shows a viable signal 141

In addition, the s-channel Drell-Yan type production, $q \bar{q}^{\prime} \rightarrow H^{ \pm} \rightarrow \tau \nu$, has been investigated by using the hadronic tau decay mode and exploiting the $\tau$ polarization $\frac{146}{16}$ For this production mode it is difficult to extract a signal since the reconstructed transverse mass distribution overlaps with the tail of the overwhelmingly large $q \bar{q}^{\prime} \rightarrow W \rightarrow \tau \nu$ background.

\subsection{The MSSM discovery potential in various benchmark scenarios}

Different benchmark scenarios have been proposed for the interpretation of MSSM Higgs boson searches 147 In the MSSM, the masses and couplings of the Higgs bosons depend, in addition to $\tan \beta$ und $m_{A}$, on the SUSY parameters through radiative corrections. In a constrained model, where unification of the $\mathrm{SU}(2)$ and $\mathrm{U}(1)$ gaugino masses is assumed, the most relevant parameters are $A_{t}$, the trilinear coupling in the stop sector, the Higgs mass parameter $\mu$, the gaugino mass term $M_{2}$, the gluino mass $m_{g}$ and a common scalar mass $M_{S U S Y}$. Instead of the parameter $A_{t}$, the stop-mixing parameter $X_{t}:=A_{t}-\mu \cot \beta$ can be used. In particular the phenomenology of the light Higgs bosons $h$ depends on the SUSY scenario, for which the following have been considered in a recent study 148 
1. $m_{h}$-max scenario: the SUSY parameters are chosen such that for each point in the $\left(m_{A}, \tan \beta\right)$ parameter space a Higgs boson mass close to the maximum possible value is obtained. For fixed $M_{2}, \mu, m_{S U S Y}$ and $m_{g}$ this is achieved by adjusting the value of $X_{t}$. This scenario is similar to the maximal mixing scenario discussed above.

2. No mixing scenario: in this scenario vanishing mixing in the stop sector is assumed, i.e., $X_{t}=0$. This scenario typically gives a small mass for the lightest CP-even Higgs boson $h$ and is less favourable for the LHC.

3. Gluophobic scenario: the effective coupling of the light Higgs boson $h$ to gluons is strongly suppressed for a large area of the $\left(m_{A}, \tan \beta\right)$-plane. This requires large mixing in the stop sector, leading to cancellations between top-quark and stop loops such that the production cross section for gluon fusion is strongly suppressed.

4. Small $\alpha$ scenario: The parameters are chosen such that the effective mixing angle $\alpha$ between the CP-even Higgs bosons is small. This results in a reduced branching ratio into $b \bar{b}$ and $\tau \tau$ for large $\tan \beta$ and intermediate values of $m_{A}$.

The parameters for the four scenarios are summarized in Table $2 \frac{148}{14}$ For a given point in parameter space, couplings and branching ratios of the Higgs bosons have been calculated using the program described in Ref. 149, which is based on a two-loop diagrammatic approach in an onshell renormalization scheme 11 In this calculation, the full one-loop radiative corrections and all dominant two-loop corrections are included.

Table 2: Values of the SUSY parameters as used in Ref. 148 for the four benchmark scenarios.

\begin{tabular}{l|c|c|c|c|c} 
& $\begin{array}{c}m_{S U S Y} \\
\left(\mathrm{GeV} / \mathrm{c}^{2}\right)\end{array}$ & $\begin{array}{c}\mu \\
\left(\mathrm{GeV} / \mathrm{c}^{2}\right)\end{array}$ & $\begin{array}{c}M_{2} \\
\left(\mathrm{GeV} / \mathrm{c}^{2}\right)\end{array}$ & $\begin{array}{c}X_{t} \\
\left(\mathrm{GeV} / \mathrm{c}^{2}\right)\end{array}$ & $\begin{array}{c}m_{g} \\
\left(\mathrm{GeV} / \mathrm{c}^{2}\right)\end{array}$ \\
\hline$m_{h}$-max & 1000 & 200 & 200 & 2000 & 800 \\
No mixing & 1000 & 200 & 200 & 0 & 800 \\
Gluophobic & 350 & 300 & 300 & -750 & 500 \\
Small $\alpha$ & 800 & 2000 & 500 & -1100 & 500 \\
\hline
\end{tabular}

In the evaluation of the discovery potential for the light Higgs boson $h$ all production modes, i.e., the gluon fusion, the vector boson fusion as well as the associated $b \bar{b} h, t \bar{t} h$ and $W h$ production have been used. The decay modes $h \rightarrow \gamma \gamma, q q h \rightarrow q q \tau \tau, q q h \rightarrow q q W W, b \bar{b} h \rightarrow b b \mu \mu, t \bar{t} h$ with $h \rightarrow b \bar{b}$ and $W h \rightarrow \ell \nu b \bar{b}$ have been considered. The discovery potential for the light CP-even Higgs boson $h$ for the individual channels in the four benchmark scenarios is shown in Fig. 31 for the ATLAS experiment alone, assuming an integrated luminosity of $30 \mathrm{fb}^{-1}$.

The study shows that already at low luminosity the full $\left(m_{A}, \tan \beta\right)$-plane can be covered in all benchmark scenarios considered, apart from a region at small $m_{A}$. However, in that particular area the searches for heavier Higgs bosons have sensitivity, such that at least one MSSM Higgs boson would be discovered at the LHC already with a moderate integrated luminosity of $30 \mathrm{fb}^{-1}$ (see Fig. 32 and discussion below). In the area not yet excluded by the LEP data, the light CPeven Higgs boson is almost guaranteed to be discovered in all four scenarios via the vector boson fusion channels. For an integrated luminosity of $30 \mathrm{fb}^{-1}$ the discovery potential in the large $m_{A}$ region is dominated by the vector boson fusion channel with $h \rightarrow \tau \tau$. In the $m_{h}$-max scenario, the $q q h \rightarrow q q W W$ channel also contributes. For small $m_{A}$ and large $\tan \beta$, the $b b h$ associated production with $h \rightarrow \mu \mu$ dominates. Since many complementary channels are available at the LHC, the loss in sensitivity due to suppressed couplings in certain benchmark scenarios can be compensated for by other channels. In the small $\alpha$ scenario, for example, the effect of the suppressed branching ratio into $\tau$ leptons (visible in the region $\tan \beta>20$ and $200<m_{A}<300 \mathrm{GeV} / \mathrm{c}^{2}$ ) is nicely compensated for by the $h \rightarrow W W$ contribution. For large integrated luminosities, the $h \rightarrow \gamma \gamma$ and $h \rightarrow Z Z^{*} \rightarrow 4 \ell$ channels provide additional sensitivity.

For the heavier Higgs bosons, the production cross sections and decay branching ratios are similar for the various benchmark scenarios, in particular for large values of $m_{A}$. Therefore, a similar discovery potential as presented in Sections 7.2 and 7.3 and illustrated in Fig. 29] is expected for all scenarios considered. 

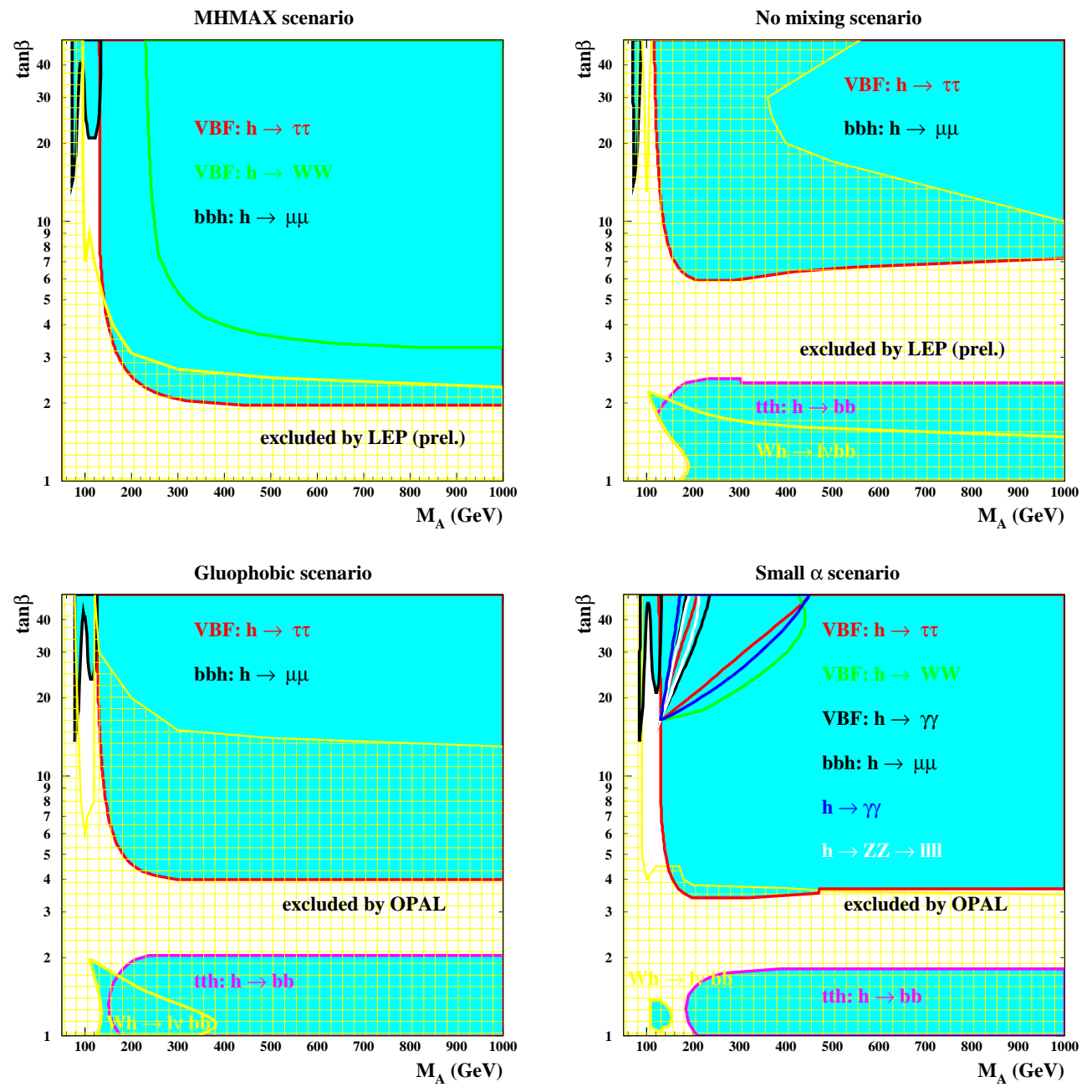

Figure 31: The $5 \sigma$ discovery contours for the light $C P$ even Higgs boson $h$ in the $\left(m_{A}, \tan \beta\right)$-plane after collecting $30 \mathrm{fb}^{-1}$ in the ATLAS experiment for the four benchmark scenarios considered: the $m_{h}$-max scenario, the no mixing scenario, the gluophobic and small $\alpha$ scenario (see text for details). The cross-hatched yellow region is excluded by searches at LEP (from Ref. 148). 

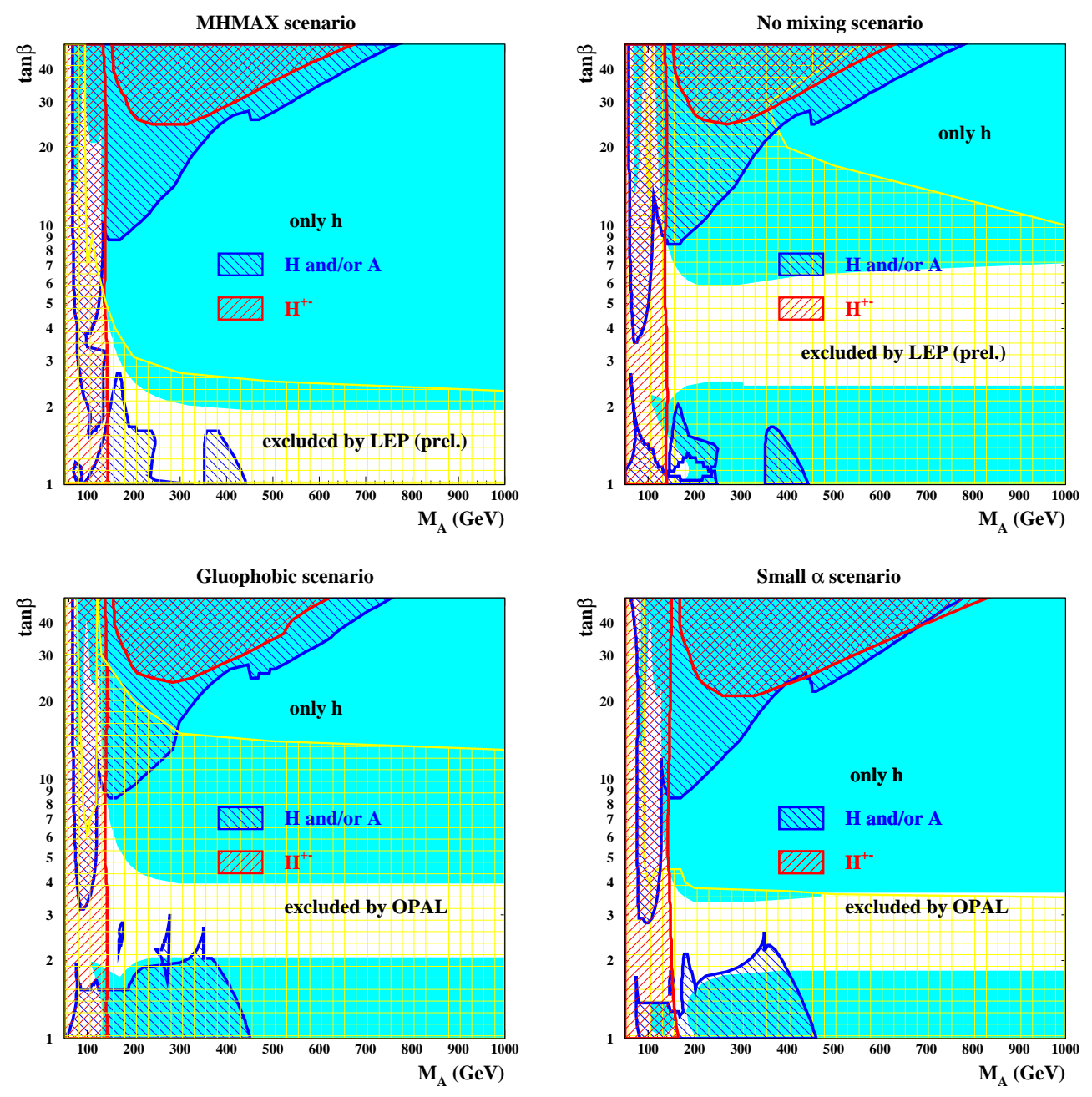

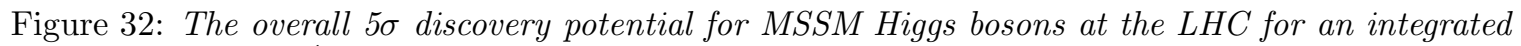
luminosity of $30 \mathrm{fb}^{-1}$ in the four benchmark scenarios (see text). In the shaded area (cyan) only the light CP-even Higgs boson $h$ can be observed. In the blue left-hatched area the heavy neutral Higgs bosons $H$ and/or $A$, and in the red right-hatched area the charged Higgs bosons $H^{ \pm}$can be detected. The cross hatched yellow region is excluded by searches at LEP (from Ref. 148). 

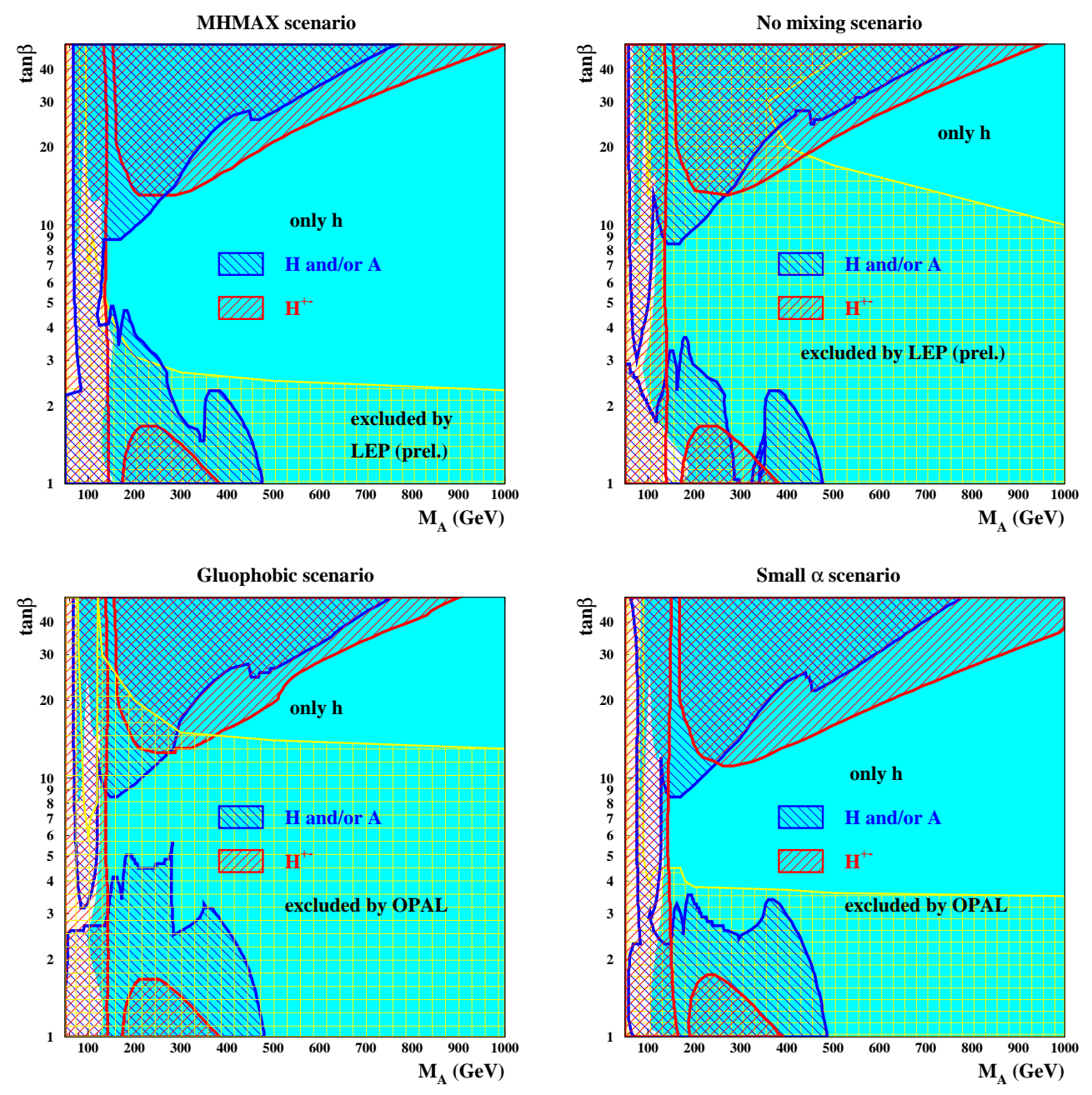

Figure 33: The same as Fig. [32, but for an integrated luminosity of $300 \mathrm{fb}^{-1}$ (except for the $A / H \rightarrow$ $\tau \tau$ decay mode, for which an integrated luminosity of $30 \mathrm{fb}^{-1}$ has been assumed) (from Ref. 148). 

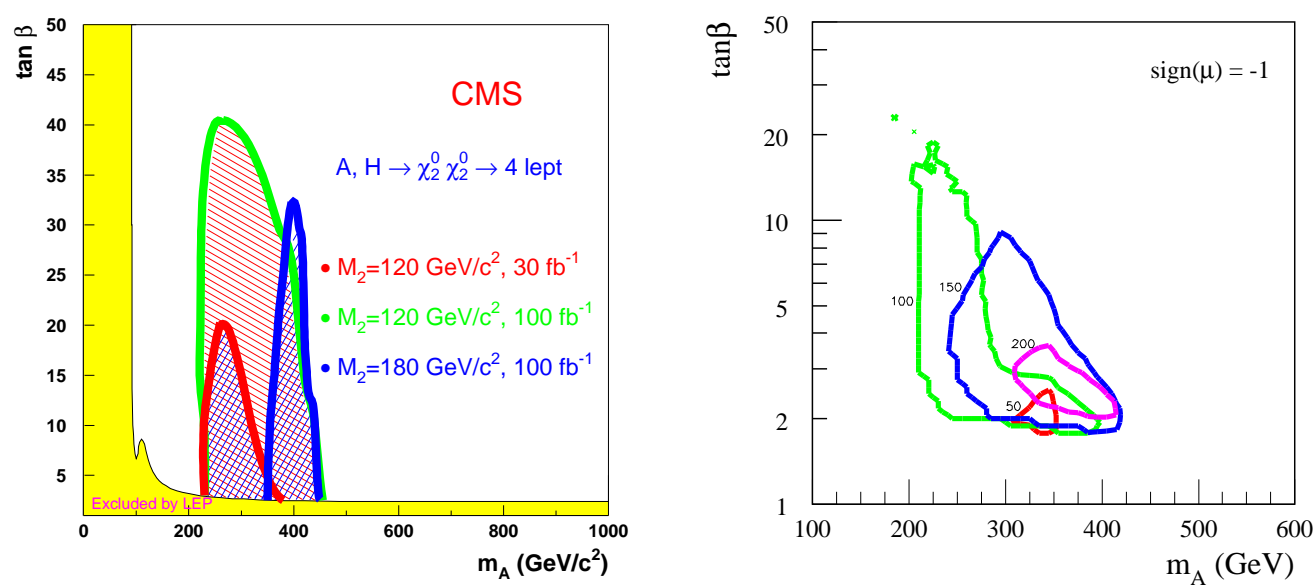

Figure 34: (Left) The $5 \sigma$ discovery contours in the $\left(m_{A}, \tan \beta\right)$-plane for $H / A \rightarrow \chi_{2}^{0} \chi_{2}^{0} \rightarrow \chi_{1}^{0} \ell \ell \chi_{1}^{0} \ell \ell$ decays for different SUSY parameters and integrated luminosities (from Ref. 69). (Right) The $5 \sigma$ discovery contours in the $\left(m_{A}\right.$, tan $\left.\beta\right)$-plane for $H / A \rightarrow \chi_{2}^{0} \chi_{2}^{0} \rightarrow \chi_{1}^{0} \ell \ell \chi_{1}^{0} \ell \ell$ in the $m S U G R A$ scenario for fixed $m_{0}=50,100$ and $200 \mathrm{GeV} / \mathrm{c}^{2}, A_{0}=0$ and $\mu<0$. The parameter $m_{1 / 2}$ has been scanned in the range between 100 and $300 \mathrm{GeV} / \mathrm{c}^{2}$. An integrated luminosity of $300 \mathrm{fb}^{-1}$ is assumed (from Ref. [68).

The overall discovery potential for the four scenarios is presented in Figs. 32 and 33 for integrated luminosities of 30 and $300 \mathrm{fb}^{-1}$, respectively. Already with a modest integrated luminosity of $30 \mathrm{fb}^{-1}$, the full parameter space can be covered for all benchmarks. However, in the region of moderate $\tan \beta$ and large $m_{A}$ only one MSSM Higgs boson, the Standard Model-like Higgs boson $h$, can be discovered, even if a large integrated luminosity of $300 \mathrm{fb}^{-1}$ is assumed. The exact location of that region in the parameter plane depends on the details of the model considered. A further increase in integrated luminosity will only marginally reduce that region.121] As discussed in Section [7.5 some sensitivity to heavier Higgs bosons might, however, be provided via their decays into SUSY particles.

Finally it should be noted that Higgs boson decays into Standard Model particles can be strongly suppressed in certain MSSM scenarios. An example has been presented in Ref. 150, where the light Higgs boson decays predominantly into light bottom squarks leading to multijet final states. In such scenarios the detection of a light Higgs boson might be difficult at hadron colliders.

\subsection{The interplay between the Higgs sector and SUSY particles}

SUSY particles have an impact on the Higgs boson discovery potential via their appearance in the decay chain (mostly for $\mathrm{H}$ and $\mathrm{A}$ ) and in loops (mostly for production via gluon fusion and for $H \rightarrow \gamma \gamma$ decays) ${ }^{151}$ In particular, the heavy Higgs bosons $H, A$ and $H^{ \pm}$could be detected via their decays into neutralinos and charginos, using multilepton final states. Due to present experimental constraints, the decay of the light Higgs boson $h$ into the lightest SUSY particles is kinematically forbidden over a large fraction of the constrained MSSM parameter space. Instead, this Higgs boson could appear at the end of the decay cascade of SUSY particles, for example in the decay $\chi_{2}^{0} \rightarrow \chi_{1}^{0} h$, where $\chi_{1}^{0}$ and $\chi_{2}^{0}$ are the lightest and second lightest neutralinos.

\subsubsection{Search for the heavy MSSM Higgs bosons in SUSY decay modes}

As discussed in Section 7.4 the heavy MSSM Higgs bosons cannot be detected via their decays into Standard Model particles in the parameter range of intermediate $\tan \beta$ and large $m_{A}$. It has been shown that decays into charginos and neutralinos can be used in some areas of SUSY parameter

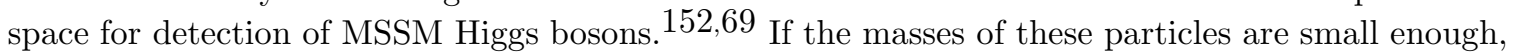
the branching ratios for the decays $H / A \rightarrow \chi_{2}^{0} \chi_{2}^{0}$ and $H^{ \pm} \rightarrow \chi_{2,3}^{0} \chi^{ \pm}$are sizeable. If in addition sleptons are light, these decays can be searched for in multilepton final states via the decay chain 


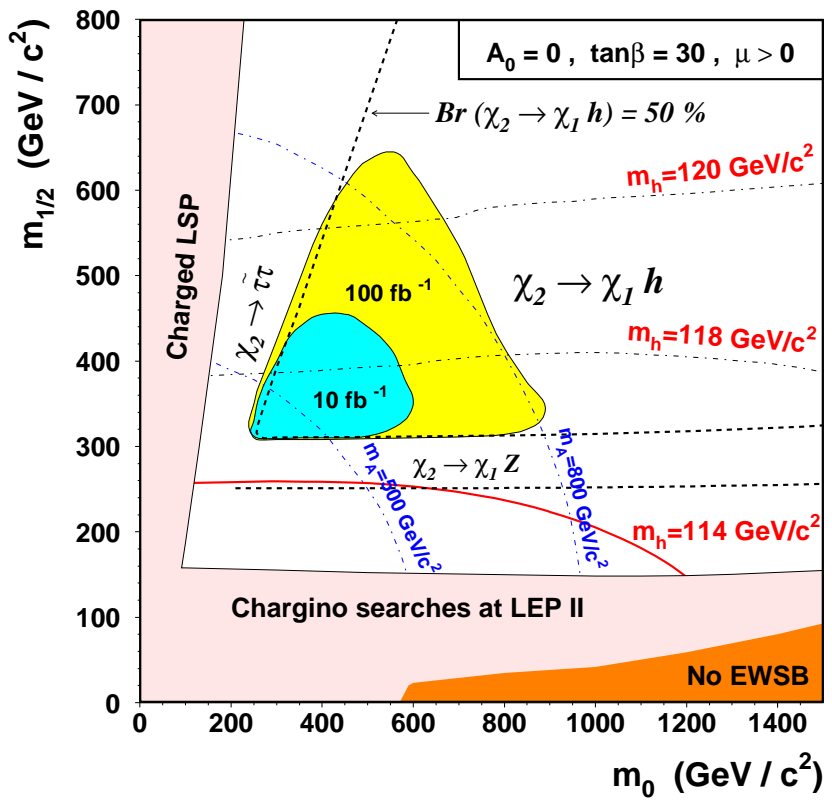

Figure 35: The $5 \sigma$ discovery contours in the $\left(m_{0}, m_{1 / 2}\right)$-plane for the CP-even Higgs bosons $h$ via the decay $h \rightarrow b \bar{b}$ from $\chi_{2}^{0} \rightarrow \chi_{1}^{0} h$ decays in squark and gluino cascades for mSUGRA scenarios with $\tan \beta=30, A_{0}=0$ and $\mu>0$ for 10 and $100 \mathrm{fb}^{-1}$. The regions excluded by experimental and theoretical constraints are indicated as well (from Ref. 69).

$\chi_{2}^{0} \rightarrow \tilde{\ell} \ell \rightarrow \chi_{1}^{0} \ell \ell$. Due to the invisible neutralinos, the Higgs boson mass reconstruction is only possible if the masses of $\chi_{1}^{0}$ and $\tilde{\ell}$ are known, for example from the analysis of first or second generation squark cascade decays 153

In the search for the decay channel $H / A \rightarrow \chi_{2}^{0} \chi_{2}^{0} \rightarrow \chi_{1}^{0} \ell \ell \chi_{1}^{0} \ell \ell$, lepton isolation, a large missing transverse energy and a jet veto are used to suppress the Standard Model backgrounds from $Z Z, Z b \bar{b}$, $Z c \bar{c}$ and $t \bar{t}$ production as well as the background from SUSY $(\tilde{q}, \tilde{g})$ production 15269 The discovery reach depends strongly on the choice of the SUSY parameters. Examples for the $5 \sigma$-discovery reach are shown in Fig. 34(left).

A similar analysis has been performed within the more constrained Minimal Supergravity (mSUGRA) 154 scenario. This model is determined by five parameters: common masses $m_{0}$ and $m_{1 / 2}$ for scalars (squarks, sleptons and Higgs bosons) and gauginos and Higgsinos at the GUT scale, a common trilinear Higgs-sfermion-sfermion coupling $A_{0}$, as well as $\tan \beta$ and the sign of the Higgs mass parameter $\mu$. Using renormalization group equations, the masses and mixings of all SUSY and Higgs particles are determined at the electroweak scale. The parameter space has been scanned for fixed values of $m_{0}=50,100,150,200$ and $250 \mathrm{GeV} / \mathrm{c}^{2}$ in the range of $m_{1 / 2}=100-300 \mathrm{GeV} / \mathrm{c}^{2}$ and $\tan \beta=$ 1.5 - 50 with $A_{0}=0.68$ The resulting $5 \sigma$ discovery contours are shown in Fig. 34(right), projected onto the $\left(m_{A}, \tan \beta\right)$-plane, for fixed values of $m_{0}$, a negative sign of $\mu$ and assuming an integrated luminosity of $300 \mathrm{fb}^{-1}$.

In the search for charged Higgs bosons, three-lepton final states from the decay chain $g b \rightarrow H^{ \pm} t$, $H^{ \pm} \rightarrow \chi_{2,3}^{0} \chi_{1,2}^{ \pm}$and $\chi_{2}^{0} \rightarrow \chi_{1}^{0} \ell \ell, \chi_{1}^{ \pm} \rightarrow \chi_{1}^{0} \ell \nu$ can be exploited 155 The accompanying top quark is required to decay hadronically. The discovery potential in this channel is limited to a small region of parameter space where sleptons are light and $|\mu| \leq 150 \mathrm{GeV} / \mathrm{c}^{2}$, i.e., close to the lower limit set by the LEP experiments.

\subsubsection{Search for $h \rightarrow b \bar{b}$ in SUSY cascade decays}

The lightest Higgs boson might appear at the end of decay cascades of SUSY particles. One copious production source may be the decay of the second lightest neutralino into the lightest neutralino, $\chi_{2}^{0} \rightarrow \chi_{1}^{0} h$. The former is produced with large rates in the decays of squarks and gluinos. In R-parity conserving SUSY models the lightest Higgs boson $h$ is in this decay mode accompanied by missing 
transverse energy, carried away by the lightest SUSY particle. The presence of missing transverse energy and several energetic jets from the squark or gluinos cascades can be used to obtain a sample that consists mainly of events containing SUSY particles. In this case, the discovery of the Higgs boson $h$ in its dominant decay mode $h \rightarrow b \bar{b}$ becomes possible, without requiring the presence of an additional lepton.

Both collaborations, ATLAS and CMS, have studied the observability of the $h \rightarrow b \bar{b}$ signal in the mSUGRA parameter space 6869156 As an example, the $5 \sigma$ discovery contours, as obtained in Ref. 69, are shown in Fig. [35] in the $\left(m_{0}, m_{1 / 2}\right)$-plane with $A_{0}=0, \tan \beta=30$ and $\mu>0$ for integrated luminosities of 10 and $100 \mathrm{fb}^{-1}$.

\subsubsection{Search for heavy MSSM Higgs bosons in SUSY cascade decays}

In a recent study four possible sources for detection of heavy MSSM Higgs bosons in SUSY cascade decays have been considered! 156

- Cascade decays of squarks and gluinos via the heavy chargino $\chi_{2}^{ \pm}$and neutralinos $\chi_{3,4}^{0}$ with subsequent decays into the lighter chargino $\chi_{1}^{ \pm}$or neutralinos $\chi_{1,2}^{0}$ and Higgs bosons:

$$
\begin{aligned}
p p \rightarrow \tilde{g} \tilde{g}, \tilde{q} \tilde{q}, \tilde{q} \tilde{g} & \rightarrow \chi_{2}^{ \pm}, \chi_{3}^{0}, \chi_{4}^{0}+X \\
& \rightarrow \chi_{1}^{ \pm}, \chi_{2}^{0}, \chi_{1}^{0}+h, H, A, H^{ \pm}+X ;
\end{aligned}
$$

- Direct decays of squarks and gluinos into the lightest chargino and the next-to-lightest neutralino, with subsequent decays into the lightest neutralino and Higgs bosons:

$$
\begin{aligned}
p p \rightarrow \tilde{g} \tilde{g}, \tilde{q} \tilde{q}, \tilde{q} \tilde{g} & \rightarrow \chi_{1}^{ \pm}, \chi_{2}^{0}+X \\
& \rightarrow \chi_{1}^{0}+h, H, A, H^{ \pm}+X ;
\end{aligned}
$$

- Direct decays of heavy stop and sbottom squarks into the lighter ones and Higgs bosons (in the case of large enough squark mass splitting):

$$
p p \rightarrow \tilde{t}_{2} \tilde{t}_{2}, \tilde{b}_{2} \tilde{b}_{2} \text {, with } \tilde{t}_{2}\left(\tilde{b}_{2}\right) \rightarrow \tilde{t}_{1}\left(\tilde{b}_{1}\right)+\mathrm{h}, \mathrm{H}, \mathrm{A} \text { or } \tilde{b}_{1}\left(\tilde{t}_{1}\right)+H^{ \pm}
$$

- Top quarks originating from SUSY particle cascades, decaying into $H^{ \pm}$bosons:

$$
p p \rightarrow \tilde{g} \tilde{g}, \tilde{q} \tilde{q}, \tilde{q} \tilde{g} \rightarrow t+X \rightarrow H^{ \pm}+X .
$$

Four representative SUSY scenarios have been discussed: squarks are considered to be either lighter or heavier than gluinos and either light gaugino- or Higgsino-like charginos and neutralinos are assumed. Using a fast simulation of the performance of the CMS detector, signals of heavy neutral (charged) Higgs bosons have been reconstructed in the $b \bar{b}(\tau \nu)$ decay mode. Backgrounds from both Standard Model and SUSY processes have been taken into account. It has been demonstrated that light Higgs bosons $\left(m_{\Phi} \lesssim 200-250 \mathrm{GeV} / \mathrm{c}^{2}\right)$ appearing in the cascade decays can be detected with a significance exceeding $5 \sigma$ in some representative MSSM scenarios. As an example, in Fig. 36 the reconstructed $b \bar{b}$ invariant mass is shown for the SUSY parameter point with $m_{\tilde{g}}=1200 \mathrm{GeV} / \mathrm{c}^{2}$, $m_{\tilde{q}}=800 \mathrm{GeV} / \mathrm{c}^{2}, M_{2}=350 \mathrm{GeV} / \mathrm{c}^{2}$ and $\mu=150 \mathrm{GeV} / \mathrm{c}^{2}$. For large $M_{2}$ values there is sufficient phase space for the decay of the heavier neutralinos and charginos, with masses $\sim M_{2}$, into the lighter Higgsino states, with masses $m_{\chi^{ \pm}} \sim m_{\chi_{1}^{0}} \sim m_{\chi_{2}^{0}} \sim|\mu|$, and Higgs particles with masses $m_{\Phi} \lesssim 200 \mathrm{GeV} / \mathrm{c}^{2}$. In the distribution two mass peaks resulting from the decays of the light Higgs boson $h$ and the heavier $H$ and $A$ are visible above the backgrounds. The discovery potential at that parameter point for heavy Higgs bosons is shown in Fig. 37 for an integrated luminosity of $100 \mathrm{fb}^{-1}$. In this scenario, the Higgs bosons $A, H\left(H^{ \pm}\right)$can be detected via the cascade decays for masses up to $m_{A} \sim 220 \mathrm{GeV} / \mathrm{c}^{2}\left(200 \mathrm{GeV} / \mathrm{c}^{2}\right)$ for all $\tan \beta$.

As discussed in Ref. 156, this study is not meant to be exhaustive, but should rather be considered as a preliminary investigation of a few representative scenarios to illustrate the discovery potential via cascade decays. In particular, a more detailed experimental simulation for a larger number of 


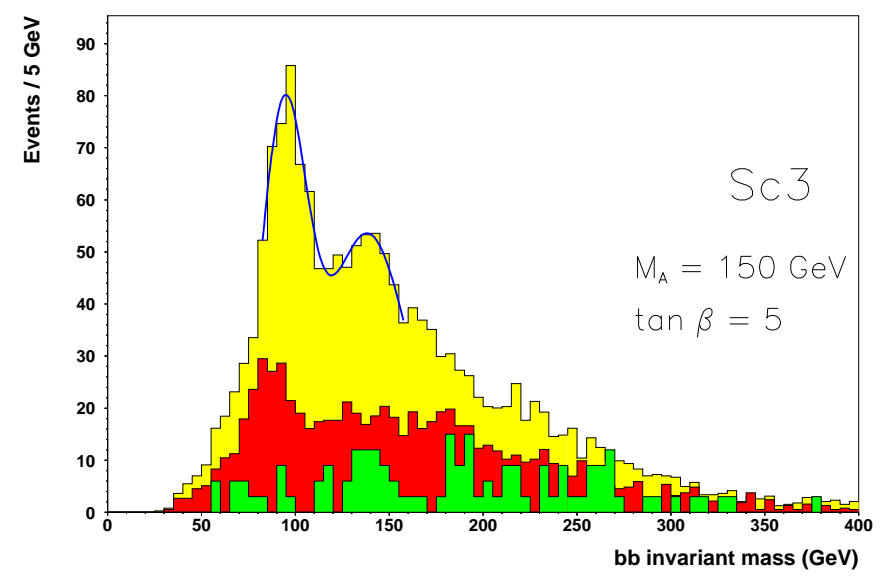

Figure 36: The reconstructed $b \bar{b}$ invariant mass spectrum for a SUSY scenario with $M_{2}=$ $350 \mathrm{GeV} / \mathrm{c}^{2}, \mu=150 \mathrm{GeV} / \mathrm{c}^{2}, m_{\tilde{g}}=1200 \mathrm{GeV} / \mathrm{c}^{2}$ and $m_{\tilde{q}}=800 \mathrm{GeV} / \mathrm{c}^{2}$ in a simulation of the CMS experiment, assuming an integrated luminosity of $30 \mathrm{fb}^{-1}$. The two mass peaks result from decays of the lighter $h$ and the heavier H/A Higgs bosons. They are shown on top of the backgrounds

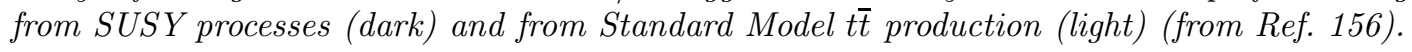

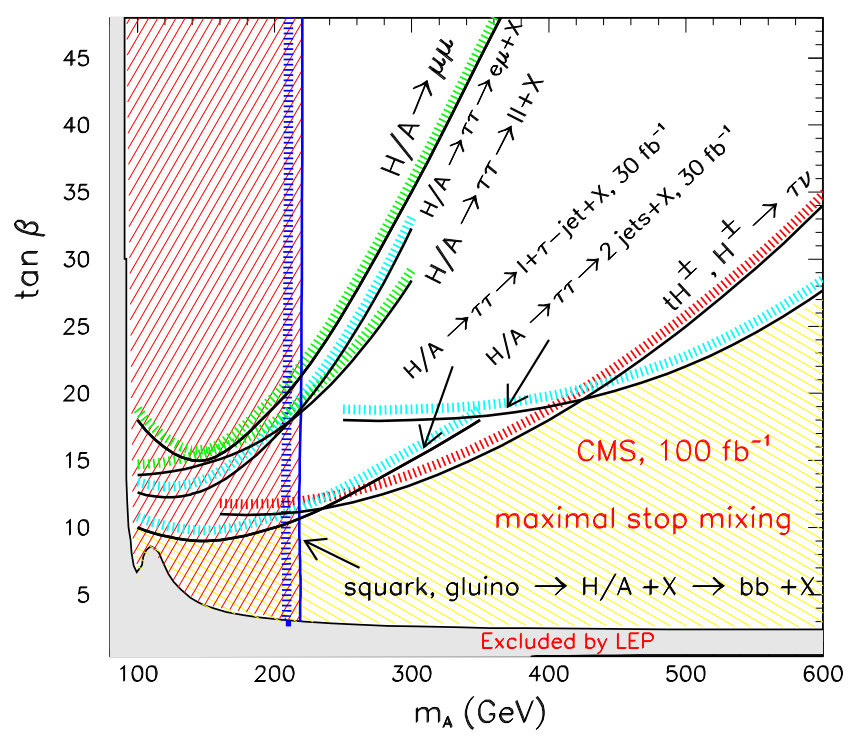

Figure 37: The $5 \sigma$ discovery contours for MSSM Higgs bosons in the $\left(m_{A}\right.$, tan $\left.\beta\right)$-plane for an integrated luminosity of $100 \mathrm{fb}^{-1}$ for the SUSY parameter point with $m_{\tilde{g}}=1200 \mathrm{GeV} / \mathrm{c}^{2}, m_{\tilde{q}}=$ $800 \mathrm{GeV} / \mathrm{c}^{2}, M_{2}=350 \mathrm{GeV} / \mathrm{c}^{2}$ and $\mu=150 \mathrm{GeV} / \mathrm{c}^{2}$. In the region with $m_{A}<220 \mathrm{GeV} / \mathrm{c}^{2}$ the heavier Higgs bosons $H$ and $A$ can be detected in cascade decays of squarks and gluinos (from Ref. 156). 
SUSY parameter points is necessary and a discussion of systematic uncertainties on the backgrounds and their impact on the signal extraction needs to be performed. In addition to the $b \bar{b}$ decay mode, also the $\tau \tau$ decay mode should be considered for heavy neutral Higgs bosons. As discussed in Section 7.6.3 the ratio of the two signals could contribute to establish the SUSY nature of a Higgs boson signal in cascade decays.

It should be stressed that the cascade processes will be extremely useful to measure the couplings of supersymmetric particles to Higgs bosons, which again would be an essential ingredient to reconstruct the parameters of the underlying SUSY model in a global fit. If cascade decays via heavier neutralinos are kinematically allowed and the relevant branching ratios are sufficiently large, the decay chain $\chi_{3,4}^{0} \rightarrow h, H, A+\chi_{1}^{0} \rightarrow b \bar{b}+\chi_{1}^{0}$ provides important information to reconstruct the masses of the heavier neutralinos, which in turn helps to constrain the SUSY model significantly.

\subsection{Determination of MSSM parameters}

Assuming that non-Standard Model Higgs bosons will be discovered at the LHC, it is important to extract the parameters of the underlying model. For this, all relevant measurements from searches for SUSY and Higgs particles will be taken into account in a global fit. The masses, production rates and branching ratios of the observed Higgs bosons are expected to contribute significantly to constrain the model. The accuracy which can be reached in the measurement of these input parameters to the global fit is discussed in the following.

\subsubsection{Measurement of the Higgs boson masses}

The precision of the mass measurement of MSSM Higgs bosons has been determined under the same assumptions as described in Section 6.1. While in addition to statistical uncertainties experimental systematic uncertainties on the background subtraction and on the knowledge of the absolute energy scale have been included, no theoretical errors have been considered. As in the case of the Standard Model, lepton and photon final states provide the highest precision for the Higgs boson mass measurements.

\section{The mass of the lightest CP-even Higgs boson}

The light CP-even Higgs boson $h$ can be detected in the $h \rightarrow \gamma \gamma$ decay mode over the full mass range of interest, i.e., between $\sim 90$ and $\sim 135 \mathrm{GeV} / \mathrm{c}^{2}$. For an integrated luminosity of $300 \mathrm{fb}^{-1}$, a precision of the mass measurement of the order of $0.1-0.5 \%$ can be achieved 68 The larger value is reached for parameter values close to the $5 \sigma$ discovery contour. A mass reconstruction is also possible in the vector boson fusion mode $q q h \rightarrow q q \tau \tau$ using the collinear approximation (see Section [5.2), however, the precision is about an order of magnitude worse.

In the $h \rightarrow b \bar{b}$ channel, the mass measurement is limited by the systematic error of $\pm 1 \%$ on the jet energy scale once signal rates are above a few hundred events. Such a signal rate will be achieved already for an integrated luminosity of only $60 \mathrm{fb}^{-1}$ over a large region of parameter space.

\section{The masses of the heavier neutral Higgs bosons}

For $m_{A} \lesssim 2 m_{t}$ and small values of $\tan \beta$, the mass of the $H$ boson can be determined in the $H \rightarrow Z \widetilde{Z}^{(*)} \rightarrow 4 \ell$ and $H \rightarrow h h \rightarrow b \bar{b} \gamma \gamma$ decay modes (see Fig. 291). Assuming an integrated luminosity of $300 \mathrm{fb}^{-1}$, a precision of the order of $0.1-0.3 \%$ for the $4 \ell$ and of about $1 \%$ for the $b \bar{b} \gamma \gamma$ channel can be achieved. ${ }^{68}$ While in the latter case the measurement is limited by systematic uncertainties, the range in the $4 \ell$ channel results from the strong variation of the signal rate with $\tan \beta$ over the discovery region.

The pseudoscalar Higgs boson $A$ can be discovered for small $\tan \beta$ in the $A \rightarrow Z h \rightarrow \ell \ell b \bar{b}$ and in the $A \rightarrow \gamma \gamma$ decay mode. The expected precision of the mass measurement is of the order of $1-2 \%$ for $\ell \ell b \bar{b}$ and of the order of $0.1 \%$ for $\gamma \gamma$ final states.

For large values of $\tan \beta$, the heavy Higgs bosons $H$ and $A$ will be discovered in the $\tau \tau$ and $\mu \mu$ decay modes. Over a large part of the parameter space they cannot be disentangled from each other, being almost degenerate in mass and having almost identical decay modes. In the region of parameter space where both $H / A \rightarrow \tau \tau$ and $H / A \rightarrow \mu \mu$ decays are observable, the precision of the 
mass measurement is determined by the $\mu \mu$ decay mode and is estimated to be at the level of $0.1 \%$. For moderate values of $\tan \beta$, where the discovery reach of the $\tau \tau$ channel extends further than that of the $\mu \mu$ channel, the precision is degraded to $1-7 \%$.

\section{The mass of the charged Higgs boson}

Both the $H^{ \pm} \rightarrow \tau \nu$ and $H^{ \pm} \rightarrow t b$ decay modes can be used to extract information on the mass of the charged Higgs boson. In $\tau \nu$ decays the mass is determined from the shape of the transverse mass distribution (see Fig. (30) using a likelihood technique.157141 For an integrated luminosity of $300 \mathrm{fb}^{-1}$, the precision of the mass measurement varies between 0.8 and $1.8 \%$ for charged Higgs boson masses in the range between 200 to $500 \mathrm{GeV} / \mathrm{c}^{2}$ !141158 In this estimate, uncertainties on the shape and on the rate of the background and on the energy scale are taken into account. Due to the larger backgrounds, the precision of the mass measurement is found to be worse in the $t b$ decay mode. 141

\subsubsection{Measurement of $\tan \beta$}

It has been suggested to determine the MSSM parameter $\tan \beta$ from the measurement of the production cross section of heavy neutral and charged MSSM Higgs bosons. ${ }^{159}$ At large $\tan \beta$ the cross sections are approximately proportional to $\tan ^{2} \beta$, however, loop corrections involving SUSY particles modify this behaviour. Due to potentially large radiative corrections to the bottom-Yukawa coupling, the results obtained from cross section measurements in this way correspond to a measurement of an effective parameter $\tan \beta_{\text {eff }}{ }^{[160}$ The extraction of the fundamental $\tan \beta$ parameter requires additional knowledge of the sbottom and gluino masses as well as of the Higgs boson mass parameter $\mu$.

The method has been applied by the ATLAS 158 and more recently by the CMS collaboration. 161 In the latter analysis, the precision of the $\tan \beta$ measurement has been determined by taking into account a systematic uncertainty of $\pm 20 \%$ on the next-to-leading order cross sections and of $\pm 3 \%$ on the branching ratios. Since the cross section depends on the Higgs boson mass, the uncertainty on the mass measurement has also been included. In order to determine the sensitivity of the $\tan \beta$ measurement to SUSY parameters, they have been varied in the range of $\pm 20 \%$ around their nominal values $\left(M_{2}=200 \mathrm{GeV} / \mathrm{c}^{2}, \mu=300 \mathrm{GeV} / \mathrm{c}^{2}, M_{S U S Y}=1 \mathrm{TeV} / \mathrm{c}^{2}\right.$ and $\left.A_{t}=2450 \mathrm{GeV} / \mathrm{c}^{2}\right)$. Over the region of parameter space where a discovery is possible, such a variation changes the cross section by at most $11 \%$, which leads to a $\pm 6 \%$ uncertainty on $\tan \beta ! 161$ For the determination of the experimental numbers, a $2-3 \%$ uncertainty on signal efficiencies, mainly related to uncertainties in $\tau$ - and b-tagging, and a $\pm 5 \%$ uncertainty on the luminosity have been assumed.

In the analysis, all combinations of tau decay channels $\left(\ell \ell, \ell \tau_{\text {had }}\right.$ and $\left.\tau_{\text {had }} \tau_{\text {had }}\right)$ have been used to extract the final measurement. In the parameter range close to the $5 \sigma$ discovery contour, where the signal rate is smallest, a statistical uncertainty of the order of $11-12 \%$ for $\tan \beta$ is found. In Fig. 38 the statistical and the combined statistical plus systematic uncertainty on $\tan \beta$ is shown as a function of $m_{A}$ for $\tan \beta=10,20,30$ and 40, assuming an integrated luminosity of $30 \mathrm{fb}^{-1}$. The total uncertainty ranges from 12 to $19 \%$ depending on $\tan \beta$ and $m_{A}$.

As mentioned above, also the cross section measurement will be used in a global fit together with other relevant measurements to determine the SUSY parameters simultaneously.

\subsubsection{Sensitivity to SUSY corrections via Higgs boson decay rates}

It has been proposed to use the ratio of charged Higgs boson decay rates, $R=\mathrm{BR}\left(H^{ \pm} \rightarrow \tau \nu\right) / \mathrm{BR}\left(H^{ \pm} \rightarrow \mathrm{tb}\right)$, to discriminate between supersymmetric and non-supersymmetric 2-Higgs-doublet models. 162 In the MSSM, Higgs boson couplings to down-type fermions receive large $\tan \beta$-dependent quantum corrections. Extensive theoretical analyses of one-loop corrections to both neutral and charged Higgs boson decay widths have been performed!160163 Depending on the parameters of the SUSY model, the value of $R$ might be changed significantly by supersymmetric radiative corrections. 162 Using a simulation of the ATLAS performance it has been shown that $R$ can be measured in the process $g b \rightarrow H^{ \pm} t$ (see Section 7.3 ). Assuming an integrated luminosity of $300 \mathrm{fb}^{-1}$, the accuracy on $R$ has been estimated to be of the order of $12-14 \%$ for $\tan \beta=50$ and $300<m_{H^{ \pm}}<500 \mathrm{GeV} / \mathrm{c}^{2}$. A 


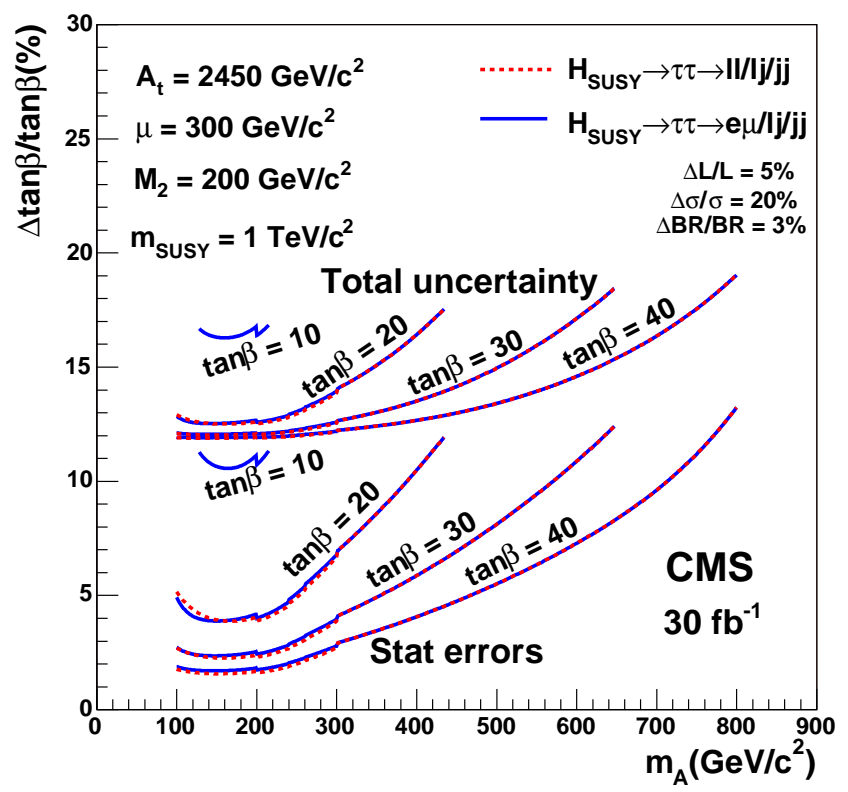

Figure 38: Relative uncertainty on the $\tan \beta$ measurement in the CMS experiment assuming an integrated luminosity of $30 \mathrm{fb}^{-1}$. The uncertainty is given for fixed values of tan $\beta$ as a function of $m_{A}$ including statistical (lower curves) and statistical plus systematic uncertainties (upper curves) (from Ref. 161).

full detector simulation and a study of systematic uncertainties on the background shape for the $H^{ \pm} \rightarrow t b$ channel still need to be carried out.

In the context of a global SUSY fit, a measurement of $R$ constitutes another important contribution from measurements in the Higgs sector.

\subsection{Search for an invisibly decaying Higgs boson}

Some extensions of the Standard Model predict Higgs bosons that decay into stable neutral weakly interacting particles. In supersymmetric models, for example, Higgs bosons can decay, in some regions of the parameter space, with a large branching ratio into the lightest neutralinos or gravitinos. 164165 In models with an enlarged symmetry breaking sector, Higgs bosons can decay into light weakly interacting scalars. 166167168 Invisible Higgs boson decays can also appear in models with large extra dimensions 169170 or if massive neutrinos of a fourth generation exist 171

In a collider detector, such decays would lead to invisible final states and triggering and detection would only be possible if the Higgs boson is produced in association with other particles. Searches have been performed at the $e^{+} e^{-}$collider LEP in the $Z H$ associated production mode. ${ }^{172}$ Since the full beam energy is absorbed in the collision, energy and momentum conservation can be used to calculate the mass of the invisibly decaying object (missing mass). Since no evidence for an invisible Higgs boson has been found, a lower limit on its mass of $114.4 \mathrm{GeV} / \mathrm{c}^{2}$ has been placed, assuming Standard Model couplings in the production and a branching ratio into invisible final states of $100 \%$ $(B R(H \rightarrow i n v)=1){ }^{172}$ Although a large fraction of the SUSY parameter space with invisible Higgs decays is excluded in the constrained MSSM (given the mass limits on the lightest neutralino from LEP experiments), invisible decays could be enhanced if gaugino mass unification is abandoned! 165

At the LHC, the search for invisibly decaying Higgs bosons is much more difficult, since the missing mass technique cannot be applied. Higgs bosons can be searched for in the associated production modes with vector bosons $(W H, Z H), t \bar{t}$-pairs $(t \bar{t} H)$ and jets (vector boson fusion mode $q q H)$. All three processes have already been suggested in the literature and feasibility studies have been performed! 173174175 In a very recent paper, the discovery potential in the $Z H$ and $q q H$ 
channels has been re-assessed and the associated production of a Higgs boson with a high- $P_{T}$ jet has been considered in addition. $\frac{176]}{1 n}$ all cases missing transverse energy is used as a key signature for the presence of an invisible Higgs boson decay.

\subsubsection{Search in $W H$ and $Z H$ associated production}

In the associated production with a vector boson, the $Z H$ process with $Z \rightarrow \ell \ell$ has been identified to be the most promising one, since the search in the $W H$ production mode is plagued with large backgrounds from inclusive $W$ production $\left[\frac{173}{T}\right.$ The dominant backgrounds to the $Z H$ process come from vector boson pair production, i.e., $Z Z \rightarrow \ell \ell \nu \nu, W Z \rightarrow \ell \nu \ell \ell$ (where the lepton from the $W$ decay is not identified or is outside of the detector acceptance) and $W W \rightarrow \ell \nu \ell \nu$ production. After basic lepton requirements, the missing transverse energy spectrum is still dominated by the background processes, however, the signal-to-background ratio improves with increasing $E_{T}$. Requiring that $E_{T}$ is larger than $75 \mathrm{GeV}$ leads to a signal-to-background ratio of $0.25 \frac{176}{16}$ It is claimed that an invisibly decaying Higgs boson could be discovered with a $5 \sigma$ significance with an integrated luminosity of $30 \mathrm{fb}^{-1}$ for Higgs boson masses up to $\sim 160 \mathrm{GeV} / \mathrm{c}^{2}$, assuming a Standard Model-like $h Z Z$ coupling and $B R(H \rightarrow i n v)=1$. The simulation of this process is currently being performed by the experimental collaborations.

Since evidence for a signal can only be claimed from an excess of events above the Standard Model backgrounds, their uncertainty needs to be taken into account in the determination of the signal significance (see also discussion at the end of Section [7.7.2).

\subsubsection{Search in $t \bar{t} H$ associated production}

In the search for an invisibly decaying Higgs boson in the associated $t \bar{t} H$ production, one of the top quarks is required to decay leptonically. This ensures that the events can be reliably triggered using the standard lepton triggers. The dominant backgrounds have been identified to be $t \bar{t}$ production and the irreducible $t \bar{t} Z$ production, with $Z \rightarrow \nu \nu ! 174$ Due to the large production cross section at the LHC, the $t \bar{t}$ background contributes significantly. In the signal selection the hadronically decaying top quark is reconstructed and two b-tags are required. In addition, the transverse mass of the $\left(\ell-\not P_{T}\right)$-system is required to be large, typically larger than $150 \mathrm{GeV} / \mathrm{c}^{2}$. This requirement rejects a large fraction of the $t \bar{t}$ background for which the transverse mass is expected to show a peak at the $\mathrm{W}$ mass ${ }^{174}$ The residual $t \bar{t}$ background results mainly from double leptonic decays of the $t \bar{t}$ system, where the second lepton $(e, \mu, \tau)$ is not identified or is outside the detector acceptance. The $\tau$ contribution in the residual $t \bar{t}$ background sample, to which both leptonic and hadronic tau decays contribute, amounts to about $70 \% ! 177$ As in the $Z H$ search, the $E_{T}$ distribution is used to establish evidence for an invisibly decaying Higgs boson. Requiring $\not_{T}>150 \mathrm{GeV}$, a signal-to-background ratio varying between 0.39 (for $m_{H}=120 \mathrm{GeV} / \mathrm{c}^{2}$ ) and 0.09 (for $m_{H}=200 \mathrm{GeV} / \mathrm{c}^{2}$ ) has been found 177 Assuming an integrated luminosity of $30 \mathrm{fb}^{-1}$, about 45 signal events are expected for $m_{H}=120 \mathrm{GeV} / \mathrm{c}^{2}$ with a $E_{T}$ cut at $150 \mathrm{GeV}$. Based on these studies, it has been concluded that a detection of an invisibly decaying Higgs boson in the associated $t \bar{t}$ production should be possible for Higgs boson masses up to $\sim 200 \mathrm{GeV} / \mathrm{c}^{2}$, assuming an integrated luminosity of $100-200 \mathrm{fb}^{-1}$. For an integrated luminosity of $30 \mathrm{fb}^{-1}$, the reach is expected to be comparable to that of the $Z H$ channel.

However, it must be stressed that in both the $Z H$ and the $t \bar{t} H$ search no signal peak is observable. A signal is claimed from an excess of events above the expectations from Standard Model processes. No systematic uncertainties on the knowledge of these backgrounds in the extreme phase space region with large $E_{T}$ have been considered. In addition, detector effects might lead to non-Gaussian tails in the $E_{T}$ distribution such that the backgrounds might be larger than anticipated so far. The experimental collaborations are studying these channels and addressing in particular the question of the uncertainty on the backgrounds and on the $E_{T}$ measurement.

\subsubsection{Search in vector boson fusion}

More recently, it has been proposed to use the vector boson fusion process $p p \rightarrow q q H \rightarrow q q+i n v$ to search for invisibly decaying Higgs bosons $\frac{175}{17}$ In contrast to the two processes discussed above, the production cross section is larger, however, triggering these events is more difficult. 


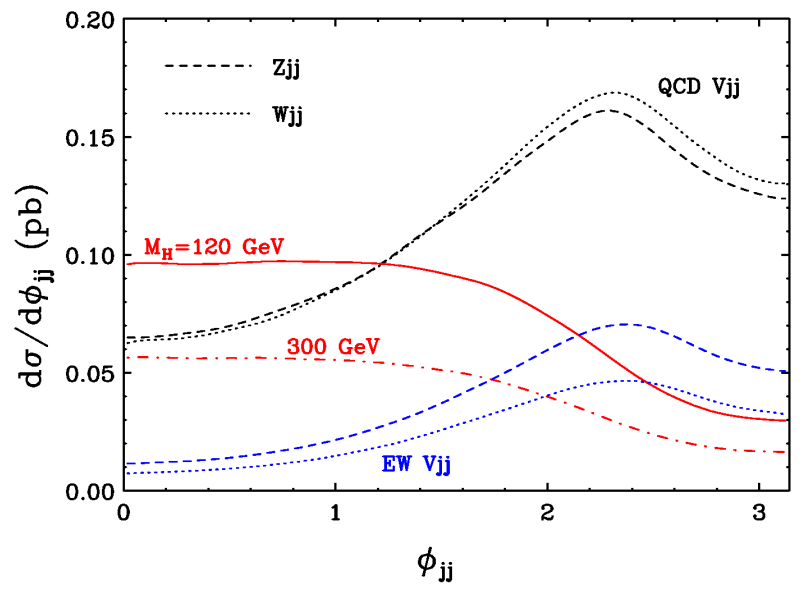

Figure 39: Distribution of the azimuthal angle separation $\left(\phi_{j j}\right)$ between the two tagging jets for the $W j j$ and $Z j j$ backgrounds from $Q C D$ and electroweak production and Higgs boson signals with $m_{H}$ $=120$ and $300 \mathrm{GeV} / \mathrm{c}^{2}$. Results are shown after applying the jet tag requirements and the cut $\mathbb{E}_{T}>$ $100 \mathrm{GeV}$ (from Ref. 175).

The experimental signature consists of two high- $P_{T}$ forward jets with a large rapidity separation and a high dijet invariant mass, accompanied by large missing transverse energy. In the analysis of Ref. [175] the forward jets are selected using similar criteria as in the vector boson fusion analyses discussed in Section 5.2 The $P_{T}$ thresholds have been raised to $40 \mathrm{GeV} / \mathrm{c}$ and the cut on the invariant mass of the two jets to $1200 \mathrm{GeV} / \mathrm{c}^{2}$. The missing transverse energy is required to be larger than $100 \mathrm{GeV}$. The Standard Model backgrounds are dominated by the $Z j j$, with $Z \rightarrow \nu \nu$, and the $W j j$, with $W \rightarrow \ell \nu$, processes. In order to reach a better discrimination between signal and the residual background, an additional cut on the azimuthal separation $\Delta \phi_{j j}<1$ between the two tag jets has been suggested! 175 The coupling structure of the Higgs boson to vector bosons favours Higgs boson emission opposite in azimuth to both tagging jets, which leads to small values of $\Delta \phi_{j j}$. The expected $\Delta \phi_{j j}$ distributions for signal and background processes are shown in Fig. 39

In Ref. [175] it has been proposed to use this distribution to constrain the $W j j$ and $Z j j$ backgrounds. At the LHC, sizeable samples of $W j j$ and $Z j j$ samples with identified leptonic vector boson decays will be available. Applying the same jet tag requirements will allow to normalize the backgrounds in the region $\Delta \phi_{j j}>1$ and to predict the background in the signal region $\left(\Delta \phi_{j j}<1\right)\left[\frac{175}{17}\right.$ Taking into account the normalization uncertainty, it has been concluded that with data corresponding to integrated luminosities of $10 \mathrm{fb}^{-1}\left(100 \mathrm{fb}^{-1}\right)$ an invisibly decaying Higgs boson with $B R(H \rightarrow i n v)=1$ can be detected with a $5 \sigma$ significance for masses up to $480 \mathrm{GeV} / \mathrm{c}^{2}$ $\left(770 \mathrm{GeV} / \mathrm{c}^{2}\right)$.

These promising results motivated a study using a more detailed simulation of the ATLAS detector 178 Similar cuts as used in the parton level analysis have been applied. In addition to the tag-jet requirements, jet veto and lepton veto cuts are applied. Events are rejected if jets with $P_{T}>20 \mathrm{GeV} / \mathrm{c}$ in the rapidity region between the tag jets or identified leptons are found. After applying all cuts, a signal cross section of the order of $60 \mathrm{fb}$ is expected for an invisibly decaying Higgs boson with $m_{H}=130 \mathrm{GeV} / \mathrm{c}^{2}$ and $B R(H \rightarrow i n v)=1$. For both the $Z j j$ and $W j j$ backgrounds, cross sections of the order of $120 \mathrm{fb}$ are expected. In comparison to these backgrounds, the contribution from QCD jet production with $E_{T}>100 \mathrm{GeV}$ is expected to be small. Performing the background normalization as described above, it is expected that the $Z j j$ background in the signal region can be predicted with a total uncertainty of $6 \%(4 \%)$ with data corresponding to an integrated luminosity of $10 \mathrm{fb}^{-1}\left(30 \mathrm{fb}^{-1}\right) ! 178$ Similarly, the $W j j$ background can be predicted with an accuracy of $\pm 3 \%(2.5 \%)$ for $10 \mathrm{fb}^{-1}\left(30 \mathrm{fb}^{-1}\right)$.

To quantify the ATLAS potential for discovery of an invisibly decaying Higgs boson, the variable $\xi^{2}=B R(H \rightarrow i n v) \cdot \sigma_{q q \rightarrow q q H} /\left(\sigma_{q q \rightarrow q q H}\right)_{S M}$ has been introduced $\frac{178}{17}$ The second term accounts for a possible suppression of the production cross section as compared to the Standard Model value. 


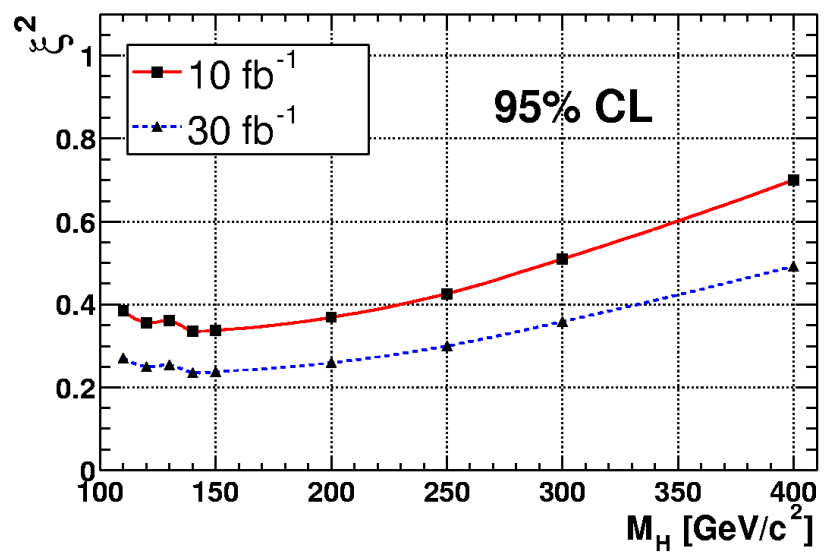

Figure 40: $\xi^{2}$ values (see text) which can be excluded with a confidence level of $95 \%$ for integrated luminosities of 10 and $30 \mathrm{fb}^{-1}$ as a function of the Higgs boson mass (from Ref. 178).

In Fig. 40 the $\xi^{2}$ values which can be excluded with a confidence level of $95 \%$ are shown for various integrated luminosities as a function of the Higgs boson mass. For an integrated luminosity of $10 \mathrm{fb}^{-1}$, it seems possible to probe $\xi^{2}$ values down to $35 \%$ for a light Higgs boson and down to $70 \%$ for a Higgs boson with $m_{H}=400 \mathrm{GeV} / \mathrm{c}^{2}$. This confirms the claim of Ref. 175 that the vector boson fusion mode extends considerably the sensitivity of the search for an invisibly decaying Higgs boson at the LHC.

As stressed in Ref. 178, these very encouraging results have been obtained with a fast detector simulation. Detector effects leading to non-Gaussian tails in the $E_{T}$ distribution cannot be estimated reliably using this simulation. Therefore, the contributions from QCD jet production might be underestimated and a more reliable estimate is needed.

The largest disadvantage of the detection of invisible Higgs boson decays in the vector boson fusion mode is the challenge to trigger on these events. An invisibly decaying Higgs boson produced via the fusion of vector bosons must be triggered exploiting the two jet plus $\mathbb{E}_{T}$ signature. Since the QCD jet production rate is huge at the LHC, high thresholds will have to be used for the jet triggers. ${ }^{92}$ At present, studies are ongoing to evaluate the design and the rates of a possible (2-jet $+E_{T}$ )-trigger! 179

\subsubsection{Summary}

The analyses presented show that the experiments at the LHC have the potential to detect invisibly decaying Higgs bosons. Different production modes can be exploited which is important to understand whether a possible excess of events with large missing transverse energy originates from Higgs boson production. In all cases evidence for a signal will be extracted from an excess of events with large $E_{T}$ above the background. For a reliable measurement a normalization of the backgrounds in the experiment will be necessary. In the associated $Z H$ and $t \bar{t} H$ production modes the expected signal cross sections are small, in the range of a few $\mathrm{fb}$, and the normalization might be affected by large uncertainties. However, those channels are nevertheless of interest since they can be reliably triggered, and in addition, the $t \bar{t} H$ channel does not rely on the vector boson coupling, which might be suppressed in certain scenarios. It should also be mentioned that all studies performed only consider backgrounds from Standard Model processes. The impact of non-Standard Model backgrounds, which might be present if Higgs bosons decay invisibly, remains to be studied. 


\section{Conclusions}

It has been demonstrated in numerous experimental studies that the experiments at the Tevatron $p \bar{p}$ collider and at the CERN Large Hadron Collider have a huge discovery potential for the Standard Model Higgs boson. If the Higgs mechanism is realized in nature the corresponding Higgs boson should not escape detection at the LHC. The full mass range can be explored and the Higgs boson can be detected in several decay modes. In addition, the parameters of the resonance can be measured with adequate precision to establish Higgs-boson like couplings to bosons and heavy fermions. For both the discovery and the parameter measurements the recently studied vector boson fusion mode plays an important role. If the Standard Model Higgs boson is light enough, the experiments at the Tevatron should already be able to observe first evidence of a signal. The significance level that can be reached at the Tevatron will depend strongly on how much luminosity can be accumulated before the LHC startup.

For the Minimal Supersymmetric Standard Model, Higgs bosons can be detected across the entire parameter space. The Tevatron experiments will be able to exclude the maximal mixing scenarios at $95 \%$ confidence level if no signal is present. At the LHC, MSSM higgs bosons can be discovered with a significance of more than $5 \sigma$ for established benchmark scenarios.

\section{Acknowledgements}

The authors would like to thank the numerous colleagues from both the theoretical and experimental community for providing such an impressive wealth of results to review. In particular, gratitude is expressed to Gregorio Bernardi, Klaus Desch, Beate Heinemann, Filip Moortgat, Sacha Nikitenko, Giacomo Polesello, Michael Spira, Guillaume Unal and Dieter Zeppenfeld for very useful discussions and comments. KJ wishes to thank his colleagues from the ATLAS Higgs working group for the excellent collaboration over many years. Particular thanks goes to Donatella Cavalli, Daniel Froidevaux, Fabiola Gianotti and Elzbieta Richter-Was for their contributions and stimulating discussions.

\section{References}

[1] S. Glashow, Nucl. Phys. 22 (1961) 579;

S. Weinberg, Phys. Rev. Lett. 19 (1967) 1264;

A. Salam, in Elementary Particle Theory, W. Svartholm, Ed., Almquist and Wiksell, Stockholm, 1968.

[2] H.D. Politzer, Phys. Rev. Lett. 30 (1973) 1346;

D.J. Gross and F.E. Wilcek, Phys. Rev. Lett. 30 (1973) 1343;

H. Fritzsch, M. Gell-Mann and H. Leutwyler, Phys. Lett. B47 (1973) 365.

[3] P.W. Higgs, Phys. Rev. Lett. 12 (1964) 132 and Phys. Rev. 145 (1966) 1156;

F. Englert and R. Brout, Phys. Rev. Lett. 13 (1964) 321;

G.S. Guralnik, C.R. Hagen and T.W. Kibble, Phys. Rev. Lett. 13 (1964) 585.

[4] For a review, see for example: J.F. Gunion, H.E. Haber, G. Kane and S. Dawson, The Higgs Hunter's Guide, Frontiers in Physics Series (Vol. 80), Addison-Wesley Publ., ISBN 0-20150935-0.

[5] H.P. Nilles, Phys. Rep. 110 (1984) 1;

H.E. Haber and G.L. Kane, Phys. Rep. 117 (1985) 75;

S.P. Martin, in Perspectives on supersymmetry, Ed. G.L. Kane, World Scientific, (1998) 1, hep-ph/9709356.

[6] ALEPH, DELPHI, L3 and OPAL Collaborations, Phys. Lett. B565 (2003) 61.

[7] The LEP Collaborations ALEPH, DELPHI, L3 and OPAL, the LEP Electroweak Working Group, the SLD Electroweak and Heavy Flavour Groups, A combination of preliminary electroweak measurements and constraints on the Standard Model, hep-ex/0312023; 
updated numbers from the LEP Electroweak Working Group, August 2004, http://lepewwg.web.cern.ch/LEPEWWG.

[8] B.W. Lee et al., Phys. Rev. Lett. 38 (1977) 883;

M. Quiros, Constraints on the Higgs boson properties from the effective potential, hepph/9703412;

A. Ghinculov and T. Binoth, Acta Phys. Polon. B30 (1999) 99.

[9] L. Maiani, G. Parisi and R. Petronzio, Nucl. Phys. B136 (1979) 115;

N. Cabibbo et al., Nucl. Phys. B158 (1979) 295;

R. Dashen and H. Neunberger, Phys. Rev. Lett. 50 (1983) 1897;

D.J.E. Callaway, Nucl. Phys. B233 (1984) 189;

M.A. Beg et al., Phys. Rev. Lett. 52 (1984) 883;

M. Lindner, Z. Phys. C31 (1986) 295.

[10] G. Altarelli and G. Isidori, Phys. Lett. B337 (1994) 141;

J.A. Casas, J.R. Espinosa and M. Quiros, Phys. Lett. B342 (1995) 171, Phys. Lett. B383 (1996) 374;

B. Grzadkowski and M. Lindner, Phys. Lett. B178 (1986) 81;

T. Hambye and K. Riesselmann, Phys. Rev. D55 (1997) 7255.

[11] S. Heinemeyer, W. Hollik and G. Weiglein, Eur. Phys. J. C9 (1999) 343;

S. Heinemeyer and G. Weiglein, J. High Energy Phys. 10 (2002) 72;

G. Degrassi, S. Heinemeyer, W. Hollik, P. Slavich and G. Weiglein, Eur. Phys. J. C28 (2003) 133.

[12] For a recent review, see: S. Heinemeyer, MSSM Higgs physics at higher orders, hep$\mathrm{ph} / 0407244$.

[13] The ALEPH, DELPHI, L3 and OPAL Collaborations and the LEP Higgs working group, Searches for the neutral Higgs bosons of the MSSM: preliminary combined results using LEP data collected at energies up to 209 GeV, CERN-EP/2001-055, hep-ex/0107030;

The ALEPH, DELPHI, L3 and OPAL Collaborations and the LEP Higgs working group, Search for neutral MSSM Higgs bosons at LEP, LHWG-Note 2004-01, Contribution to ICHEP04, Beijing (China), Aug. 2004.

[14] A. Brignole, J. Ellis, G. Ridolfi and F. Zwirner, Phys. Lett. B271 (1991) 123;

A. Brignole, Phys. Lett. B277 (1992) 313;

M.A. Diaz and H.E. Haber, Phys. Rev. D45 (1992) 4246.

[15] The ALEPH, DELPHI, L3 and OPAL Collaborations and the LEP Higgs working group, Search for charged Higgs bosons: preliminary combined results using LEP data collected at energies up to 209 GeV, CERN-EP/2000-055, hep-ex/0107031.

[16] CDF Collaboration, Phys. Rev. Lett. 79 (1997) 357;

CDF Collaboration, Phys. Rev. D62 (2000) 012004;

DØ Collaboration, Phys. Rev. Lett. 82 (1999) 4975;

DØ Collaboration, Phys. Rev. Lett. 88 (2002) 151803.

[17] CLEO Collaboration, M.S. Alam et al., Phys. Rev. Lett. 74 (1995) 2885;

R. Briere, Proc. of ICHEP98, Vancouver, Canada (1998);

ALEPH Collaboration, R. Barate et al., Phys. Lett. B429 (1998) 169.

[18] P. Gambino and M. Misiak, Nucl. Phys. B611 (2001) 338;

F.M. Borzumati and C. Greub, Phys. Rev. D58 (1998) 074004, hep-ph/9802391; Phys. Rev. D59 (1999) 057501, hep-ph/9809438;

J.A. Coarasa, J. Guasch, J. Sola and W. Hollik, Phys. Lett. B442 (1998) 326, hep-ph/9808278.

[19] M. Spira, private communication. 
[20] A. Djouadi, M. Spira and P.M. Zerwas, Phys. Lett. B264 (1991) 440;

S. Dawson, Nucl. Phys. B359 (1991) 283;

D. Graudenz, M. Spira and P.M. Zerwas, Phys. Rev. Lett. 70 (1993) 1372;

M. Spira, A. Djouadi, D. Graudenz and P.M. Zerwas, Phys. Lett. B318 (1993) 347 and Nucl. Phys. B453 (1995) 17;

S. Dawson and R.P. Kauffman, Phys. Rev. D49 (1994) 2298.

[21] R.V. Harlander, Phys. Lett. B492 (2000) 74;

R.V. Harlander, W.B. Kilgore, Phys. Rev. D64 (2001) 013015;

S. Catani, D. de Florian, M. Grazzini, JHEP 0105 (2001) 025.

[22] R.V. Harlander, W.B. Kilgore, Phys. Rev. Lett. 88 (2002) 201801.

[23] C. Anastasiou, K. Melnikov, Nucl. Phys. B646 (2002) 220.

[24] V. Ravindran, J. Smith and W.L. van Neerven, Nucl. Phys. B665 (2003) 325.

[25] M. Krämer, E. Laenen and M. Spira, Nucl. Phys. B511 (1998) 523;

see for example: R.V. Harlander, Higgs production at hadron colliders, in Proc. Hadron Collider Physics 2002, Springer Verlag (2002).

[26] R. Harlander, private communication.

[27] W. Beenakker, S. Dittmaier, M. Krämer, B. Plümper, M. Spira and P.M. Zerwas, Phys. Rev. Lett. 87 (2001) 201805;

W. Beenakker, S. Dittmaier, M. Krämer, B. Plümper, M. Spira and P.M. Zerwas, Nucl. Phys. B653 (2003) 151, hep-ph/0211352.

[28] L. Reina, S. Dawson, Phys. Rev. Lett. 87 (2001) 201804;

L. Reina, S. Dawson, D. Wackeroth, Phys. Rev. D65 (2002) 053017;

S. Dawson, L.H. Orr, L. Reina, D. Wackeroth, Phys. Rev. D67 (2003) 071503.

[29] For a review see: J. Campbell et al., Higgs boson production in association with bottom quarks, Proc. Les Houches workshop 2003, hep-ph/0405302.

[30] R.M. Barnett, H.E. Haber and D.E. Soper, Nucl. Phys. B306 (1988) 697;

F.I. Olness and W.K. Tung, Nucl. Phys. B308 (1988) 813;

D. Dicus and S. Willenbrock, Phys. Rev. D39 (1989) 751.

[31] S. Dittmaier, M. Krämer and M. Spira, Phys. Rev. D70 (2004) 074010, hep-ph/0309204.

[32] S. Dawson, C. Jackson, L. Reina and D. Wackeroth, Phys. Rev. D69 (2004) 074027.

[33] J. Campbell, R.K. Ellis, F. Maltoni and S. Willenbrock, Phys. Rev. D67 (2003) 095002, hep$\mathrm{ph} / 0204093 \mathrm{v} 2$.

[34] D. Dicus, T. Stelzer, Z. Sullivan and S. Willenbrock, Phys. Rev. D59 (1999) 094016;

C. Balazs, H.J. He and C.P. Yuan, Phys. Rev. D60 (1999) 114001.

[35] R. Harlander and W.B. Kilgore, Phys. Rev. D68 (2003) 013001.

[36] M. Carena, J.S. Conway, H.E. Haber et al., Report of the Higgs working group of the Tevatron Run II SUSY/Higgs workshop, hep-ph/0010338;

G.C. Blazey et al., Proceedings of the Run II QCD and Weak Boson Physics workshop, hepex/0005012;

V. Barger, C.E.M. Wagner et al., Report of the SUGRA working group for Run II of the Tevatron, hep-ph/0003154.

[37] D. Rainwater, M. Spira and D. Zeppenfeld, Proc. Les Houches 2001, hep-ph/0203187, http://wwwlapp.in2p3.fr/conferences/LesHouches/Houches2001.

[38] T. Han, G. Valencia, S. Willenbrock, Phys. Rev. Lett. 69 (1992) 3274. 
[39] T. Figy, C. Oleari and D. Zeppenfeld, Phys. Rev. D68 (2003) 073005, hep-ph/0306109;

E. L. Berger and J. Campbell, Phys. Rev. D70 (2004) 073011, hep-ph/0403194.

[40] T. Han, S. Willenbrock, Phys. Lett. B273 (1991) 167;

A. Djouadi, M. Spira, Phys. Rev. D62 (2000) 014004.

[41] R. Hamberg, W.L. van Neerven and T. Matsuura, Nucl. Phys. B359 (1991) 343; O. Brein, A. Djouadi and R. Harlander, Phys. Lett. B579 (2004) 149.

[42] A. Djouadi, J. Kalinowski and M. Spira, Comput. Phys. Commun. 108 (1998) 56, hep$\mathrm{ph} / 9704448$;

A. Djouadi, M. Spira and P. M. Zerwas, Z. Phys. C70 (1996) 427, hep-ph/9511344.

[43] A. Ghinculov, T. Binoth and J.J. van der Bij, Phys. Lett. B427 (1998) 343.

[44] CDF Collaboration, The CDF II Detector Technical Design Report, FERMILAB-Pub-96/390E.

[45] DØ Collaboration, The Upgraded DØ Detector, to be submitted to Nucl. Instrum. Methods A;

T. Le Compte and H.T. Diehl, Ann. Rev. Nucl. Part. Sci. 50 (2000) 71.

[46] ATLAS Collaboration, ATLAS Technical proposal, CERN/LHCC/94-43 (1994), and Technical Design Reports of the detector subsystems, CERN/LHCC, http://atlas.web.cern.ch/Atlas/internal/tdr.html.

[47] CMS Collaboration, CMS Technical proposal, CERN/LHCC 94-38 (1994), and Technical Design Reports of the detector subsystems, CERN/LHCC, http://cmsinfo.cern.ch/Welcome.html/cmsdocsite.html.

[48] R. Brun and F. Carminati, CERN Program Library Long Writeup W5013 (1993).

[49] CDF and DØ Collaborations, Results of the Tevatron Higgs Sensitivity Study, FERMILABPUB-03/320-E (2003).

[50] J. Goldstein et al., Phys. Rev. Lett. 86 (2001) 1694.

[51] CDF Collaboration, Search for New Particle $X \rightarrow b \bar{b}$ Produced in Association with $W$ Bosons at $\sqrt{s}=1.96 \mathrm{Te} V$, CDF Note 7126

DØ Collaboration, A Search for Wbb and WH production in $p \bar{p}$ collisions at $\sqrt{s}=1.96 \mathrm{TeV}$, FERMILAB-PUB-04/288-E (2004), submitted to Phys. Rev. Lett.

[52] DØ Collaboration, Search for the Higgs Boson in $H \rightarrow W W \rightarrow l \nu l \nu$ Decays at DØ in Run II, DØ Note 4387-CONF;

CDF Collaboration, Search for the Standard-Model Higgs Boson in the Decay Channel of $H \rightarrow W W \rightarrow l \nu l \nu, l=e, \mu$, with $184 \mathrm{pb}^{-1}$ of Run II Data at CDF, CDF Note 7152.

[53] M. Dittmar and H.K. Dreiner, Phys. Rev. D55 (1997) 167.

[54] T. Han, A.S. Turcot, R.-J. Zhang, Phys. Rev. D59 (1999) 093001.

[55] CDF Collaboration, Search for the WH Production Using High-P $P_{T}$ Isolated Like-Sign Dilepton Events in Run II, CDF Note 7307.

[56] C.T. Hill, Phys. Lett. B266 (1991) 419.

[57] E. Arik, M. Arik, S.A. Cetin, T. Conka, A. Mailov and S. Sultansoy, Eur. Phys. J. C26 (2002) 9 .

[58] DØ Collaboration, Search for a non-SM Light Higgs Boson in the $h \rightarrow \gamma \gamma$ Channel at DØ in Run II, DØ Note 4374-CONF.

[59] DØ Collaboration, A DØ Search for Neutral Higgs Bosons at high $\tan \beta$ in Multijet Events, DØ Note 4366-CONF. 
[60] CDF Collaboration, Phys. Rev. Lett. 86 (2001) 4472.

[61] J. Yamaoka, Uncertainties on PDF's affecting 3b/4b MSSM Higgs search, Presentation at the Tev4LHC Workshop, Fermilab, Sept. 2004.

[62] DØ Collaboration, First measurement of $\sigma(p \bar{p} \rightarrow Z) \operatorname{Br}(Z \rightarrow \tau \tau)$ at $\sqrt{s}=1.96 \mathrm{TeV}$, FERMILAB-PUB-04/381-E (2004), submitted to Phys. Rev. Lett.;

CDF Collaboration, Measurement of $\sigma \times B R(p \bar{p} \rightarrow Z \rightarrow \tau \tau)$ at $1.96 \mathrm{TeV}$, Contribution to ICHEP04, Beijing (China), Aug. 2004.

[63] CDF Collaboration, Search for MSSM Higgs Decaying to Tau Pairs, CDF Note 7161.

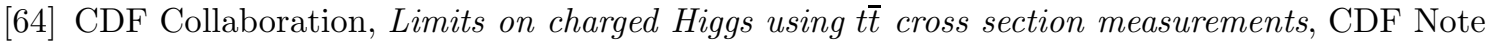
7250 .

[65] J.C. Pati and A. Salam, Phys. Rev. D10 (1974) 275;

R.N. Mohapatra and J.C. Pati, Phys. Rev. D11 (1975) 566;

G. Senjanovic and R.N. Mohapatra, Phys. Rev. D12 (1975) 1502;

T. Rizzo, Phys. Rev. D25 (1982) 1355, and Addendum ibid D27 (1983) 657.

[66] DØ Collaboration, Phys. Rev. Lett. 93 (2004) 141801;

CDF Collaboration, Phys. Rev. Lett. 93 (2004) 221802.

[67] CDF Collaboration, Search for the Quasi-Stable Doubly-Charged Higgs Using the Two-Track Signature, CDF Note 7155.

[68] ATLAS Collaboration, Detector and Physics Performance Technical Design Report, CERN/LHCC/99-15 (1999).

[69] S. Abdullin et al., Summary of the CMS potential for the Higgs boson discovery, CMS NOTE $2003 / 033$.

[70] D.L. Rainwater and D. Zeppenfeld, J. High Energy Phys. 12 (1997) 5, hep-ph/9712271.

[71] D.L. Rainwater, D. Zeppenfeld and K. Hagiwara, Phys. Rev. D59 (1999) 014037, hep$\mathrm{ph} / 9808468$

T. Plehn, D.L. Rainwater and D. Zeppenfeld, Phys. Rev. D61 (2000) 093005, hep-ph/9911385.

[72] D.L. Rainwater and D. Zeppenfeld, Phys. Rev. D60 (1999) 113004,

[Erratum-ibid. D61 (2000) 099901], hep-ph/9906218;

N. Kauer, T. Plehn, D.L. Rainwater and D. Zeppenfeld, Phys. Lett. B503 (2001) 113.

[73] T. Sjostrand, Comp. Phys. Comm. 82 (1994) 74.

[74] M.N. Dubinin, V.A. Ilyin and V.I. Savrin, Light Higgs boson signal at the LHC in the reaction $p p \rightarrow \gamma \gamma+j e t$ and $p p \rightarrow \gamma \gamma+\ell$, CMS NOTE 1997/101;

S. Abdullin et al., Phys. Lett. B431 (1998) 410.

[75] G. Martinez, E. Gross, G. Mikenberg and L. Zivkovic, Prospects for light Higgs observation in the $H \rightarrow Z Z^{*} \rightarrow b \bar{b}$ ee $(\mu \mu)$ channel at the LHC, ATLAS note, ATL-PHYS-2003-001.

[76] B. Mohn and B. Stugu, Corrections to the discovery potential for finding the Standard Model Higgs in the four lepton final state, ATLAS note, ATL-PHYS-2004-014.

[77] U. Baur and E.W.N. Glover, in Proc. of the Large Hadron Collider Workshop, Aachen 1990, Ed. G. Jarlskog and D. Rein, CERN 90-10/ECFA 90-133, Vol.II, p. 570.

[78] K. Jakobs and Th. Trefzger, Standard Model Higgs boson search for $H \rightarrow W W^{(*)} \rightarrow \ell \nu \ell \nu$ with a mass between $150-190 \mathrm{GeV} / \mathrm{c}^{2}$ at the LHC, ATLAS note, ATL-PHYS-2000-015;

D. Green et al., Search for the Standard Model Higgs boson with $m_{H} \sim 170 \mathrm{GeV} / \mathrm{c}^{2}$, in $\mathrm{W}^{+} \mathrm{W}^{-}$ decay mode, J. Phys. G26 (2000) 1751. 
[79] S.Asai et al., Prospects for the search of a Standard Model Higgs boson in ATLAS using vector boson fusion, Eur. Phys. J. C32 (2003) 209, ATLAS note SN-ATLAS-2003-024, hep$\mathrm{ph} / 0402254$.

[80] G. Azuelos et al., Search for the Standard Model Higgs Boson using Vector Boson Fusion at the LHC, Proc. Les Houches 2001, hep-ph/0203056, http://wwwlapp.in2p3.fr/conferences/LesHouches/Houches2001.

[81] V. Cavasinni, D. Costanzo and I. Vivarelli, Forward tagging and jet-veto studies for Higgs events produced via vector boson fusion, ATLAS note, ATL-PHYS-2002-008 (2002).

[82] E. Richter-Was, D. Froidevaux and L. Poggioli, ATLFAST, a fast simulation package for ATLAS, ATLAS note, ATL-PHYS-98-131.

[83] C.M. Buttar, R.S. Harper and K. Jakobs, Weak boson fusion $H \rightarrow W W^{(*)} \rightarrow \ell^{+} \ell^{-} E_{T}$ as a search mode for an intermediate mass SM Higgs boson at ATLAS, ATLAS note, ATL-PHYS2002-033;

K. Cranmer, B. Mellado, W. Quayle and Sau Lan Wu, Search for Higgs boson decays $H \rightarrow$ $W^{+} W^{-} \rightarrow \ell^{+} \ell^{-} E_{T}$ for $115<m_{H}<130 \mathrm{GeV} / c^{2}$ using vector boson fusion, ATLAS note, ATL-PHYS-2003-002;

K. Cranmer et al., Neural network based search for Higgs bosons produced via VBF with $H \rightarrow$ $W^{+} W^{-} \rightarrow \ell^{+} \ell^{-} E_{T}$ for $115<m_{H}<130 \mathrm{GeV} / c^{2}$, ATLAS note, ATL-PHYS-2003-007;

K. Cranmer et al., Analysis of VBF $H \rightarrow W W \rightarrow l \nu l \nu$, ATLAS note, ATL-PHYS-2004-019.

[84] N. Akchurin et al., Study of low mass Higgs using $p p \rightarrow q \bar{q} H$ at CMS, CMS NOTE 2002/016.

[85] M. Dittmar and A.S. Nicollerat, High mass Higgs studies using $g g \rightarrow H$ and $q q \rightarrow q q H$ at the LHC, CMS NOTE 2001/036.

[86] V. Cavasinni, D. Costanzo, E. Mazzoni and I. Vivarelli, Search for an intermediate mass Higgs boson produced via vector boson fusion in the channel $H \rightarrow W W^{(*)} \rightarrow \ell \nu$ jet jet, ATLAS note, ATL-PHYS-2002-010.

[87] F.A. Berends, W.T. Giele, K. Kuijf, B. Tausk, The VECBOS Monte Carlo programme, Fermilab-Pub-90/213-T.

[88] M. Klute, A study of the weak boson fusion with $H \rightarrow \tau \tau$ and $\tau \rightarrow e(\mu)$, ATLAS note, ATLPHYS-2002-018;

R. Mazini and G. Azuelos, Searching for $H \rightarrow \tau^{+} \tau^{-} \rightarrow l \nu_{l} \nu_{\tau}+h X$ by vector boson fusion in ATLAS, ATLAS note, ATL-PHYS-2003-004.

[89] M. Dubinin, Higgs boson signal in the reaction $p p \rightarrow \gamma \gamma$ plus two forward jets, CMS NOTE $2001 / 022$.

[90] K. Cranmer, B. Mellado, W. Quayle and Sau Lan Wu, Search for Higgs boson decay $H \rightarrow \gamma \gamma$ using vector boson fusion, ATLAS note, ATL-PHYS-2003-036.

[91] K. Cranmer et al., Prospects for Higgs searches via VBF at the LHC with the ATLAS detector, ATLAS note, ATL-PHYS-2004-005.

[92] ATLAS Collaboration, The ATLAS HLT, DAQ $\&$ DCS Technical Design Report, http://atlas-proj-hltdaqdes-tdr.web.cern.ch/atlas-proj-hltdaqdes-tdr.

[93] A. De Roeck et al., Eur. Phys. J. C25 (2002) 391, hep-ph/0207042; V.A. Khoze et al., Eur. Phys. J. C26 (2003) 429, hep-ph/0207365.

[94] M.L. Mangano et al., Phys. Lett. B556 (2003) 50, hep-ph/0210261.

[95] P.H. Beauchemin and G. Azuelos, Search for the Standard Model Higgs Boson in the $\gamma \gamma+\mathbb{E}_{T}$ channel, ATLAS note, ATL-PHYS-2004-028.

[96] A. Pukhov et al., CompHEP - a package for evaluating Feynman diagrams and integration over multi-particle phase space, INP MSU 98-41/542, hep-ph/9908288. 
[97] E. Richter-Was, Revisiting the observability of the WH and $Z H, H \rightarrow b \bar{b}$ channel in $14 \mathrm{TeV}$ $p p$ and $2 \mathrm{TeV} p \bar{p}$ collisions, ATLAS note, ATL-PHYS-2000-024.

[98] B. King, S. Maxfield and J. Vossebeld, Search for a light Standard Model Higgs in the channel $p p \rightarrow t \bar{t} H, W H, Z H$, ATLAS note, ATL-PHYS-2004-031.

[99] V. Drollinger, Th. Müller, and D. Denegri, Prospects for Higgs boson searches in the channel $W^{ \pm} H \rightarrow \ell^{ \pm} \nu b \bar{b}$, CMS NOTE 2002/006, hep-ph/0201249.

[100] V. Drollinger, Th. Müller and D. Denegri, Searching for Higgs bosons in association with top quark pairs in the $H \rightarrow b \bar{b}$ decay mode, CMS NOTE 2001/054, hep-ph/0111312.

[101] E. Richter-Was and M. Sapinski, Search for the SM and MSSM Higgs boson in the $t t H, H \rightarrow b \bar{b}$ channel, ATLAS note, ATL-PHYS-98-132;

J. Cammin and M. Schumacher, The ATLAS discovery potential for the channel $t \bar{t} H, H \rightarrow b \bar{b}$, ATLAS note, ATL-PHYS-2003-024.

[102] A. Stange, W. Marciano and S. Willenbrock, Phys. Rev. D50 (1994) 327;

J.F. Gunion and T. Han, Phys. Rev. D51 (1995) 1051.

[103] H. Baer and J.D. Wells, Phys. Rev. D57 (1998) 4446;

K. Jakobs, A study of the associated production $W H$, with $W \rightarrow \ell \nu$ and $H \rightarrow W W^{(*)} \rightarrow \ell \nu \ell \nu$, ATLAS note, ATL-PHYS-2000-008.

[104] J. Leveque, J.B. de Vivie, V. Kostioukhine and A. Rozanov, Search for the Standard Model Higgs boson in the $t \bar{t} H, H \rightarrow W W^{(*)}$ channel, ATLAS note, ATL-PHYS-2002-019.

[105] see for example: L. Brücher, R. Santos, Eur. Phys. J. C12 (2000) 87, hep-ph/9907434, and references therein.

[106] J. Campbell, K. Ellis, MCFM - Monte Carlo for FeMtobarn processes, http://mcfm.fnal.gov.

[107] S. Frixione and B. Webber, The MC@NLO Package, http://www.hep.phy.cam.ar.uk/theory/webber/MCatNLO.

[108] T. Gleisberg, S. Hoche, F. Krauss, A. Schalicke, S. Schumann and J. C. Winter, The SHERPA Monte Carlo, JHEP 0402 (2004) 056, hep-ph/0311263;

http://www.physik.tu-dresden.de/ krauss/hep/index.html.

[109] V. Drollinger and A. Sopczak, Eur. Phys. J direct C3 (2001).

[110] D. Zeppenfeld, R. Kinnunen, A. Nikitenko and E. Richter-Was, Phys. Rev. D62 (2000) 013009.

[111] M. Dührssen, Prospects for the measurement of Higgs boson coupling parameters in the mass range from $110-190 \mathrm{GeV} / \mathrm{c}^{2}$, ATLAS note, ATL-PHYS-2003-030.

[112] C.P. Buszello et al., Eur. Phys. J. C32 (2003) 209.

[113] S.Y. Choi, D.J. Miller, M.M. Mühlleitner and P.M. Zerwas, Phys. Lett. B553 (2003) 61, hep-ph/0210077.

[114] J.F. Gunion and X.G. He, Phys. Rev. Lett. 76 (1996) 4468.

[115] T. Plehn, D. Rainwater and D. Zeppenfeld, Phys. Rev. Lett. 88 (2002) 051901.

[116] M. Spira, HIGLU: a program for the calculation of the total Higgs production cross section at hadron colliders via gluon fusion including QCD corrections, hep-ph/9510347 (1995).

[117] B. Kniehl, Phys. Rep. 240 (1994).

[118] M. Dührssen et al., Phys. Rev. D70 (2004) 113009, hep-ph/0406323. 
[119] D. Dicus, C. Kao and S. Willenbrock, Phys. Lett. B203 (1988) 457;

E.W.N. Glover and J.J. van der Bij, Nucl. Phys. B309 (1988) 282;

F. Boudjema and E. Chopin, Z. Phys. C73 (1996) 85;

V.A. Ilyin et al., Phys. Rev. D54 (1996) 6717;

A. Djouadi, W. Kilian, M. Mühlleitner and P.M. Zerwas, Eur. Phys. J. C10 (1999) 27;

D.J. Miller and S. Moretti, Eur. Phys. J. C13 (2000) 459.

[120] T. Plehn, M. Spira and P.M. Zerwas, Nucl. Phys. B479 (1996) 46;

A. Djouadi, W. Kilian, M. Mühlleitner and P.M. Zerwas, Eur. Phys. J. C10 (1999) 45.

[121] G. Azuelos et al., J. Phys. G28 (2002), 2453, hep-ph/0204087.

[122] U. Baur, T. Plehn and D. Rainwater, Phys. Rev. Lett. 89 (2002) 151801;

U. Baur, T. Plehn and D. Rainwater, Phys. Rev. D68 (2003) 033003.

[123] F. Mazzucato, A. Blondel and A. Clark, Studies on the measurement of the SM Higgs selfcouplings, ATLAS note, ATL-PHYS-2002-029.

[124] U. Baur, T. Plehn and D. Rainwater, Phys. Rev. D69 (2004) 053004, hep-ph/0310056.

[125] E. Richter-Was et al., Int. J. Mod. Phys. A13 (1998) 1371.

[126] See for example: M. Carena, P.M. Zerwas (Conv) et al., Proc. of the LEP2 Workshop, G. Altarelli, T. Sjostrand and F. Zwirner (Ed), CERN 1995.

[127] D. Denegri et al., Summary of the CMS discovery potential for the MSSM SUSY Higgses, CMS NOTE 2001/032, hep-ph/0112045.

[128] S. Gonzales, E. Ros and M. Vos, Analysis of the process $p p \rightarrow b b h / A \rightarrow b b \mu \mu$ in the MSSM with $m_{A}<125 \mathrm{GeV} / \mathrm{c}^{2}$, ATLAS note, ATL-PHYS-2002-024.

[129] S. Gentile, M. Paniccia and P. Violini, Search for the supersymmetric neutral Higgs $h$ in the decay $h \rightarrow \mu \mu$ in the ATLAS detector at LHC, ATLAS note, ATL-PHYS-2003-013.

[130] J. Dai, J.F. Gunion and R. Vega, Phys. Lett. B345 (1995) 29, Phys. Lett. B387 (1996) 801.

[131] D. Cavalli and G. Negri, Extension of the study of $A / H \rightarrow \tau \tau \rightarrow \ell$ had $+\ldots$ in the high $m_{A}$ region, ATLAS note, ATLAS-PHYS-2003-009;

J. Thomas, Study of heavy MSSM Higgs bosons A/H in hadronic tau decays in ATLAS, ATLAS note, ATL-PHYS-2003-003.

[132] R. Kinnunen and A. Nikitenko, Study of $H \rightarrow \tau^{+} \tau^{-}$with hadronic $\tau$ decays in $C M S$, CMS NOTE 2003/006.

[133] E. Richter-Was, ATLAS Higgs working group, private communication.

[134] K.J.F. Gaemers and G. Hoogeveen, Phys. Lett. B146 (1984) 347;

A. Dicus, A. Stange and S. Willenbrock, Phys. Lett. B333 (1994) 126.

[135] A.C. Bawa, C.S. Kim and A.D. Martin, Z. Phys. C47 (1990) 75;

L.G. Jin, C.S. Li, R.J. Oakes and S.H. Zhu, Eur. Phys. J. C14 (2000) 91;

A. Belyaev, D. Garcia, J. Guasch and J. Sola, J. High Energy Phys. 06 (2002) 059.

[136] S.H. Zhu, Complete next-to-leading order QCD corrections to charged Higgs boson associated production with top quark at the CERN Large Hadron Collider, hep-ph/0112109;

T. Plehn, Phys. Rev. D67 (2003) 014018;

E.L. Berger, T. Han, J. Jiang and T. Plehn, Associated production of a top quark and a charged Higgs boson, hep-ph/0312286.

[137] J. Gunion, H.E. Haber, F. Paige, W. Tung and S. Willenbrock, Nucl. Phys. B294 (1987) 621; D.A. Dicus, J.L. Hewett, C. Kao and T.G. Rizzo, Phys. Rev. D40 (1989) 787;

W. Hollik and S.H. Zhu, Phys. Rev. D65 (2002) 075015;

S. Willenbrock, Phys. Rev. D35 (1987) 173; 
S.H. Zhu, C.S. Li and C.S. Gao, Phys. Rev. D58 (1998) 055007;

S. Moretti and K. Odagiri, Phys. Rev. D59 (1999) 055008;

O. Brein and W. Hollik, Eur. Phys. J. C13 (2000) 175;

O. Brein, W. Hollik and S. Kanemura, Phys. Rev. D63 (2001) 095001;

A.A. Barrientos Bendezu and B.A. Kniehl, Phys. Rev. D59 (1999) 015009 and Phys. Rev. D64 (2001) 035006.

[138] C. Biscarat and M. Dosil, Charged Higgs search in top quark decays with the ATLAS detector, ATLAS note, ATL-PHYS-2003-038.

[139] M. Drees and D.P. Roy, Phys. Lett. B269 (1991) 155;

J.F. Gunion, Phys. Lett. B322 (1994) 125, hep-ph/9312201;

V.D. Barger, R.J. Phillips and D.P. Roy, Phys. Lett. B324 (1994) 236;

K. Odagiri, Phys. Lett. B452 (1999) 327;

S. Moretti and D.P. Roy, Phys. Lett. B470 (1999) 209.

[140] D.P. Roy, Phys. Lett. B277 (1992) 183 and Phys. Lett. B459 (1999) 607.

[141] K.A. Assamagan, Y. Coadou and A. Deandrea, ATLAS discovery potential for a heavy charged Higgs boson, ATLAS note, SN-ATLAS-2002-017, hep-ph/0203121.

[142] R. Kinnunen, Study of heavy charged Higgs in $p p \rightarrow t H^{ \pm}$with $H^{ \pm} \rightarrow \tau \nu$ in CMS, CMS NOTE $2000 / 045$.

[143] K.A. Assamagan and N. Gollub, The ATLAS discovery potential for a heavy charged Higgs boson in $g g \rightarrow H^{ \pm} t b$ with $H^{ \pm} \rightarrow t b$, ATLAS note, SN-ATLAS-2004-042, hep-ph/0406013.

[144] K.A. Assamagan, M. Guchait and S. Moretti, Charged Higgs bosons in the transition region $M_{H^{ \pm}} \sim m_{t}$ at the LHC, Proc. Les Houches 2003, hep-ph/0402057.

[145] M. Drees, M. Guchait and D.P. Roy, Phys. Lett. B471 (1999) 39, and references therein.

[146] S. Slabospitsky, Study of s-channel charged Higgs production in CMS, CMS NOTE 2002/010, hep-ph/0203094.

[147] M. Carena, S. Heinemeyer, C.E. Wagner and G. Weiglein, Eur. Phys. J. C26, (2003) 601.

[148] M. Schumacher, Investigation of the discovery potential for Higgs bosons in the CP-conserving MSSM with the ATLAS detector at the LHC, hep-ph/0410112;

M. Schumacher, priv. communication, ATLAS note in preparation.

[149] S. Heinemeyer, W. Hollik and G. Weiglein, Phys. Rev. D58 (1998) 091701, Phys. Lett. B440 (1998) 296, FEYNHIGGS program: http://www.feynhiggs.de.

[150] E.L. Berger, C.W. Chiang, J. Jiang, T.M.P. Tait and C.E.M. Wagner, Phys. Rev. D66 (2002) 095001, hep-ph/0205342.

[151] H. Baer, M. Bisset, X. Tata and J. Woodside, Phys. Rev. D46 (1992) 46.

[152] S. Abdullin, D. Denegri and F. Moortgat, Observability of MSSM Higgs bosons via sparticle decay modes in CMS, CMS NOTE 2001/042.

[153] M.M. Nojiri, G. Polesello and D.R. Tovey, Proposal for a new reconstruction technique for SUSY processes at the $L H C$, hep-ph/0312317.

[154] L. Alvarez-Gaume, J. Polchinski and M.B. Wise, Nucl. Phys. B221 (1983) 495;

L. Ibanez, Phys. Lett. B118 (1982) 73;

J. Ellis, D.V. Nanopolous and K. Tamvakis, Phys. Lett. B121 (1983) 123;

K. Inoue et al., Prog. Theor. Phys. 68 (1982) 927;

A.H. Chamseddine, R. Arnowitt and P. Nath, Phys. Rev. Lett. 49 (1982) 970.

[155] M. Bisset, F. Moortgat and S. Moretti, Trilepton + top signal from chargino-neutralino decays of MSSM charged Higgs bosons at the LHC, CMS NOTE 2003/004, hep-ph/0303093. 
[156] A. Datta, A. Djouadi, M. Guchait and F. Moortgat, Nucl. Phys. B681 (2004) 31, hep$\mathrm{ph} / 0303095$.

[157] M. Hohlfeld, On the determination of Higgs parameters in the ATLAS experiment at the LHC, ATLAS note, ATL-PHYS-2001-004.

[158] K.A. Assamagan and Y. Coadou, Determination of the charged Higgs mass and of tan $\beta$ with ATLAS, ATLAS note, ATL-PHYS-2001-017.

[159] J.F. Gunion et al., Higgs boson discovery and properties, Snowmass 96, hep-ph/9703330.

[160] L.J. Hall, R. Rattazi and U. Sarid, Phys. Rev. D50 (1994) 7048;

M. Carena, M. Olechowski, S. Pokorski and C.E. Wagner, Nucl. Phys. B426 (1994) 269;

M. Carena, D. Garcia, U. Nierste and C.E. Wagner, Nucl. Phys. B577 (2000) 88;

J. Guasch, P. Häflinger and M. Spira, hep-ph/0305101.

[161] R. Kinnunen et al., Measurement of the $H / A \rightarrow \tau \tau$ cross section and possible constraints on $\tan \beta$, CMS NOTE 2004/027.

[162] K.A. Assamagan, J. Guasch, S. Moretti and S. Penaranda, Determining the ratio of the $H^{+} \rightarrow$ $\tau \nu$ to $H^{+} \rightarrow t \bar{b}$ decay rates for large tan $\beta$ at the Large Hadron Collider, Proc. Les Houches 2003, hep-ph/0402212.

[163] J. Guasch, R.A. Jimenez and J. Sola, Phys. Lett. B360 (1995) 47, hep-ph/9507461;

J.A. Coarasa et al., Eur. Phys. J. C2 (1998) 373, hep-ph/9607485;

J.A. Coarasa et al., Phys. Lett. B425 (1998) 329, hep-ph/9711472;

H.E. Haber et al., Phys. Rev. D63 (2001) 055004, hep-ph/0007006. M.J. Herrero, S. Penaranda and D. Temes, Phys. Rev. D64 (2001) 115003, hep-ph/0105097;

M. Carena, H.E. Haber, H.E. Logan and S. Mrenna, Phys. Rev. D65 (2002) 055005, hepph/0106116, erratum-idid. D65 (2002) 099902;

A. Belyaev, D. Garcia and J. Sola, Nucl. Phys. Proc. Suppl. 116 (2003) 296; J. Guasch.

P. Häfliger and M. Spira, Phys. Rev. D68 (2003) 115001, hep-ph/0305101.

[164] H. Haber, D. Dicus, M. Drees and X. Tata, Phys. Rev. D37 (1987) 1367;

J.F. Gunion and H.E. Haber, Nucl. Phys. B307 (1988) 445;

K. Griest and H.E. Haber, Phys. Rev. D37 (1988) 719;

A. Djouadi et al., Phys. Lett. B376 (1996) 220;

A. Djouadi, P. Janot, J. Kalinowski and P.M. Zerwas, Phys. Lett. B376 (1996) 220;

A. Djouadi and M. Drees, Phys. Lett. B407 (1997) 243.

[165] G. Belanger et al., Nucl. Phys. B581 (2000) 3;

G. Belanger et al., Phys. Lett. B519 (2001) 93, hep-ph/0106275;

G. Belanger et al., J. High Energy Phys. 03 (2004) 12, hep-ph/0310037.

[166] R.E. Shrock and M. Suzuki, Phys. Lett. B10 (1982) 250;

L.F. Li, Y. Liu and L. Wolfenstein, Phys. Lett. B159 (1985) 45.

[167] A.S. Joshipura and J.W.F. Valle, Nucl. Phys. B397 (1993) 105.

[168] T. Binoth and J.J. van der Bij, Z. Phys. C75 (1997) 17.

[169] N. Arkani-Hamed, S. Dimopoulos and G. Dvali, Phys. Lett. B429 (1998) 263;

I. Antoniadis, N. Arkani-Hamed, S. Dimopoulos and G. Dvali, Phys. Lett. B436 (1998) 257;

K. Dienes, E. Dudas and T. Gherghetta, Phys. Lett. B436 (1998) 55 and Nucl. Phys. B537 (1999) 47.

[170] S.P. Martin and D. Wells, Phys. Rev. D60 (1999) 35006, hep-ph/9903259;

G.F. Giudice, R. Rattazzi and J.D. Wells, Nucl. Phys. B595 (2001) 250, hep-ph/0002178.

[171] K. Belotsky et al., Phys. Rev. D68 (2003) 054027, hep-ph/0210153.

[172] LEP Higgs boson working group, Searches for invisible Higgs decays, hep-ph/0107032;

DELPHI Collaboration, Eur. Phys. J. C32 (2004) 475. 
[173] D. Choudhury and D.P. Roy, Phys. Lett. B322 (1994) 368, hep-ph/9312347;

S. Frederiksen, N. Johnson, G. Kane and J. Reid, Phys. Rev. D50 (1994) 4244.

[174] J.F. Gunion, Phys. Rev. Lett. 72 (1994) 199, hep-ph/9309216.

[175] O.J.P. Eboli and D. Zeppenfeld, Phys. Lett. B495 (2000) 147.

[176] H. Davoudiasl, T. Han and H.E. Logan, Discovering an invisibly decaying Higgs at Hadron Colliders, hep-ph/0412269.

[177] B.P. Kersevan, M. Malawski and E. Richter-Was, Eur. Phys. J. C29 (2003) 541.

[178] L. Neukermans and B. Di Girolamo, Observing an invisibly decaying Higgs boson in ATLAS via vector boson fusion, ATLAS note, ATL-PHYS-2003-006.

[179] D. Cavalli et al., Proc. Les Houches 2001, hep-ph/0203056, http://wwwlapp.in2p3.fr/conferences/LesHouches/Houches2001. 